\title{
Propagation of Optical Pulses in Dynamic Media: A Time Transformation Method \\ by
}

Yuzhe Xiao

Submitted in Partial Fulfillment of the

Requirements for the Degree

Doctor of Philosophy

Supervised by

Govind P. Agrawal

The Institute of Optics

Arts, Sciences and Engineering

Edmund A. Hajim School of Engineering and Applied Sciences

University of Rochester

Rochester, New York 


\section{Biographical Sketch}

The author was born in Changsha, China in 1986. In 2004 he enrolled in the Department of Physics at the Harbin Institute of Technology, Harbin, China, for undergraduate study with a major in Optical Information Science and Technology, and got his B.S. in July, 2008. In the Fall of 2008 he enrolled in the Ph.D. program in The Institute of Optics at the University of Rochester. In the Summer of 2009 he joined the research group of Professor Govind Agrawal, where he has carried out his doctoral research on optical pulse propagation modeling. He obtained his M.S. in Optics from the University of Rochester in March 2012. 


\section{Publications}

\section{Publications related to this thesis}

1 Y. Xiao, D. N. Maywar and G. P. Agrawal, "Reflection and transmission of electromagnetic fields from a temporal boundary," Opt. Lett. 39, 574-577 (2014).

2 Y. Xiao, D. N. Maywar and G. P. Agrawal, "Time-transformation approach to pulse propagation in nonlinear dispersive media: Inclusion of delayed Raman nonlinearity," Phy. Rev. A 87063816 (2013).

3 Y. Xiao, D. N. Maywar and G. P. Agrawal, "Propagation of few-cycle pulses in nonlinear Kerr media: harmonic generation," Opt. Lett. 38, 724-726 (2013).

4 Y. Xiao, D. N. Maywar and G. P. Agrawal, "New approach to pulse propagation in nonlinear dispersive optical media," J. Opt. Soc. Am. B 29, 2958-2963 (2012).

5 Y. Xiao, G. P. Agrawal and D. N. Maywar, "Nonlinear pulse propagation: A time transformation approach," Opt. Lett. 37, 1271-1273 (2012). 
6 Y. Xiao, D. N. Maywar and G. P. Agrawal, "Optical pulse propagation in dynamic Fabry-Perot resonators," J. Opt. Soc. Am. B 28, 1685-1692 (2011).

7 Y. Xiao, G. P. Agrawal and D. N. Maywar, "Spectral and temporal changes of optical pulses propagating through time-varying linear media," Opt. Lett. 36, 505-507 (2011).

\section{Other Publications}

8 S. Mumtaz, Y. Xiao, R. J. Essiambre, and G. P. Agrawal, "Impact of random linear mode coupling on nonlinear propagation in multimode fibers," in preparation.

9 Y. Xiao, S. Mumtaz, R. J. Essiambre, and G. P. Agrawal, "Theory of intermodal four wave mixing in few-mode fibers," submitted, in review.

10 P.P. Baveja, Y. Xiao, S. Arora, G. P. Agrawal, and D. N. Maywar, "All-Optical SOA-Based Wavelength Converters with Sub-mW Pumping," IEEE Photon. Technol. Lett. 25, 78-80 (2013).

\section{Conference Presentations}

1 Y. Xiao, D. N. Maywar and G. P. Agrawal, "Pulse propagation in dynamic optical media: a time transformation approach," IEEE Photonic Society Summer Topical Meeting on Nonlinear-Optical Signal Processing, Montreal, Canada, 2014. 
2 Y. Xiao, S. Mumtaz, R. J. Essiambre, and G. P. Agrawal, "Effect of random mode coupling on intermodal four wave mixing in few-mode fibers", accepted, Optical Fiber Communication Conference (OFC) 2014.

3 Y. Xiao, D. N. Maywar and G. P. Agrawal, "Propagation of few-cycle pulses in nonlinear Kerr media: harmonic generation," in Conference on Lasers and Electro-Optics (CLEO), San Jose, CA, USA, 2013.

4 Y. Xiao, D. N. Maywar and G. P. Agrawal, "Time-transformation approach to nonlinear pulse propagation: Kerr and delayed Raman response," in CLEO, San Jose, CA, USA, 2013.

5 Y. Xiao, D. N. Maywar and G. P. Agrawal, "A new approach to pulse propagation in nonlinear optical media," in CLEO, San Jose, CA, USA, 2012.

6 Y. Xiao, G. P. Agrawal and D. N. Maywar, "Adiabatic wavelength conversion in travelling-wave and resonant photonic structures," in Integrated Photonics Research, Silicon and Nanophotonics (IPRSN), Toronto, Canada, 2011.

7 Y. Xiao, D. N. Maywar and G. P. Agrawal, "Dynamic Frequency Shifts in Photonic Structures," in Frontiers in Optics (FiO)/Laser Science XXVI (LS), Rochester, NY, USA, 2010 . 


\section{Acknowledgments}

The last six years at Rochester would definitely make a memorable time in my life. Though there were challenges and frustrations from time to time, most of the time was made up of mostly joyful moments on my journey in pursuit of a Ph.D. degree. Without the guidance and help from many people, my journey would not have been so pleasurable. I would like to take this chance to thank a number of people who have contributed to making this possible.

The person showing up in the first place of this list is my thesis advisor Prof. Govind P. Agrawal. I want to thank him for his guidance, support and encouragement for the last five years. He is a knowledgeable scholar, and he gave me guidance from all aspects of my research. He is also an excellent teacher, and taking his classes was really an enjoyment. He had great patience in helping me with each of my papers, and the past five years in his group was a quality time in my life. Most important of all, he is a great man and also a mentor for life.

I am indebted to Prof. Drew N. Maywar from Rochester Institute of Technology (RIT), who gave me lot of help both in my theoretical and experimental research 
parts throughout my whole graduate study. I worked closely with him in developing my time transformation method, and his comments were critical to the birth of this method. He also gave me guidance when I performed experiments in his Photonic System Lab at RIT.

I would like to thank Dr. René-Jean Essiambre from Bell Labs, Acatel-Lucent. He gave me valuable suggestions when I began working on a project related to spatial division multiplexing (SDM) using multimode and multi-core fibers with him for my last year. I learned a lot about optical fiber communication systems from him, and also learned a lot from his enthusiasm in science and research.

I am also grateful to the members of my research group. Brian Daniel helped me get through the first transition year into the group. I learned a lot of hands on experimental skills from Prashant Baveja. I had several useful discussions with Sami Mumtaz about the SDM project. I also want to thank Néstor Crisóstomo, Shaival Buch, and Brent Plansinis for many insightful discussions.

I want to thank my parents, who gave me birth to this world and have always been there throughout the lows and the highs with unconditional love and support.

Finally, I would like to thank the reminder of my thesis committee, Prof. Miguel A. Alonso and Prof. Gary W. Wicks for their comments and suggestions on both my thesis proposal and this document. 


\section{Abstract}

A novel time transformation method is proposed for studying the propagation of optical pulses through dynamic media (including both dynamic linear and nonlinear media) whose refractive indices change with time. This method is developed by extending the delta-function concept of the impulse response of a dielectric medium and incorporating a physical picture of the time delay introduced by such a medium.

The first part of this thesis is devoted to dynamic linear media, and to providing an explanation to the recent discovered phenomena of "adiabatic wavelength conversion" (AWC). A universal formula is derived for the output electric field after an optical pulse has propagated through such a dynamic linear medium. Besides the frequency shift, new important aspects related to AWC, such as modifications to pulse width and amplitude, are also found. By appropriate modifications, the time transformation method can also be extended to resonators. A detailed numerical study of AWC in a Fabry-Perot resonator is performed, and the impact of different parameters, such as the pulse width, cavity photon lifetime, relative speed of index change, and the detuning of input frequency, on AWC in a cavity is investigated. 
A time-dependent refractive index change represents a temporal boundary for the electric field. The reflection and transmission of electromagnetic waves from such a temporal boundary is also discussed in the first part of this thesis. It is shown that two different physical processes are involved in this process. One process is related to impedance mismatch, while the other results from temporal scaling related to a sudden change in the speed of light at the temporal boundary.

The second part of this thesis is devoted to nonlinear optical media, which are also dynamic in the sense that their refractive indices change with light intensity. Conceptually, the time transformation method shows that the effect of propagation through a non-dispersive Kerr medium (with an instant nonlinearity) is to change the relative spacing and duration of various temporal electric field slices of the pulse, which manifest as self-phase modulation and self-steepening in the spectral and temporal domains, respectively. The impact of Raman (delayed) nonlinearity and chromatic dispersion are appropriately included into the time transformation method through a generalized integration. Using the time transformation method, the dependence of the soliton fission, intrapulse Raman scattering, and dispersive-wave generation on the pulse width is investigated. Finally, carrier wave shocking and harmonic generation are studied considering the nonlinear propagation of few-cycle pulses.

The proposed time transformation method works directly with the electric field, and does not make the usually adopted slowly varying envelop approximation. It can be applied to pulses of arbitrary widths and should find applications in a variety of 
fields, such as ultrafast optics and nonlinear optics. 


\section{Contributors and Funding Sources}

This work was supervised by a dissertation committee consisting of Professor Govind P. Agrawal (advisor), Miguel A. Alonso, and Gary W. Wicks of The Institute of Optics, University of Rochester and Professor Drew N. Maywar of the Department of Electrical, Computer, and Telecommunication Engineering Technology, Rochester Institute of Technology. The work presented in this thesis comes from papers 1-7 in "Publications related to this thesis", where the author was the only student that has worked on these projects. Materials from papers 8-10 in "Other Publications" are not included in this thesis. Graduate study was supported by a research award from National Science Foundation (NSF ECCS-1041982) and an Agnes M. and George Messersmith Fellowship from the University of Rochester. 


\section{Table of Contents}

Biographical Sketch ii

Publications $\quad$ iii

Acknowledgments $\quad$ vi

Abstract viii

Contributors and Funding Sources $\quad$ xi

List of Figures $\quad$ xvi

1 Introduction 1

1.1 Historical overview . . . . . . . . . . . . . . . . . . 1

1.2 Brief discussion of problems studied . . . . . . . . . . . 3

1.3 Thesis outline . . . . . . . . . . . . . . . . 5 
2 Background and Review of Past Work $\quad 8$

2.1 Dynamic linear media . . . . . . . . . . . . . . 8

2.2 Nonlinear media . . . . . . . . . . . . . . . . . . . . 30

3 Time Transformation Method $\quad 42$

3.1 Linear system theory . . . . . . . . . . . . . . . 43

3.2 Solution of Maxwell's equations . . . . . . . . . . . . . . 45

3.3 Time transformation method . . . . . . . . . . . . . . . 47

3.4 Inclusion of chromatic dispersion . . . . . . . . . . . . . . 53

4 Adiabatic Wavelength Conversion in Dynamic Linear Media 57

4.1 Analytical results for dynamic linear media . . . . . . . . . . . 58

4.2 Adiabatic wavelength conversion . . . . . . . . . . . . . 61

4.3 Impact of time-dependent $n(t) \ldots \ldots \ldots \ldots$. . . . . . . 67

4.4 Reflection and transmission of electromagnetic waves at a temporal

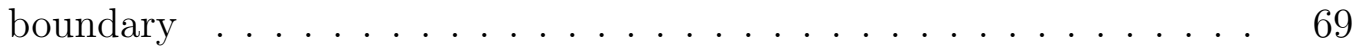

5 Adiabatic Wavelength Conversion in Dynamic Linear Resonators 79

5.1 Impulse response for dynamic resonators . . . . . . . . . . . . . 80

5.2 Propagation of short optical pulses . . . . . . . . . . . 83

5.3 Propagation of long optical pulses . . . . . . . . . . . . . . 89

5.4 Variations of external parameters . . . . . . . . . . . . . . 92 
6 Kerr Media: Instantaneous Nonlinearity

6.1 Time transformation by a Kerr medium . . . . . . . . . . . . . 104

6.2 Non-dispersive Kerr medium . . . . . . . . . . . . . . . . . . . . . . 109

6.3 Dispersive Kerr medium . . . . . . . . . . . . . . . . . . . . . . 114

7 Raman Media: Delayed Nonlinearity 124

7.1 Time-transformation by a Raman medium . . . . . . . . . . 125

7.2 Comparison with the NLS equation approach . . . . . . . . . 128

7.3 Propagation of few-cycle pulses $\ldots \ldots \ldots \ldots \ldots$

7.4 Propagation of single-cycle pulses . . . . . . . . . . . . . 137

8 Kerr Nonlinearity for Few-Cycle Pulses 143

8.1 Nonlinearity without averaging . . . . . . . . . . . . . . 144

8.2 Carrier wave shocking and harmonic generation $\ldots \ldots \ldots \ldots$

8.3 Impact of finite Kerr response time . . . . . . . . . . . . . . 147

8.4 Inclusion of dispersion: soliton propagation $\ldots \ldots \ldots \ldots \ldots$

9 Conclusions and Final Thoughts

A.1 Extension to pulse envelopes . . . . . . . . . . . . . . 157

A.2 Application to a nonlinear dispersive medium . . . . . . . . . . . 159 


\section{List of Figures}

2.1 Schematic illustration of the AWC process. AWC happens because of a time-dependent refractive index of the medium inside a resonator, and the shift in wavelength is linear proportional to change in the refractive

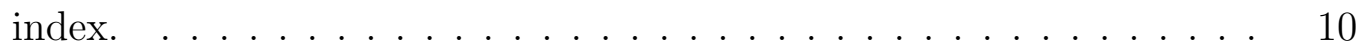

2.2 Comparison of spatial and temporal refractive index discontinuities. (a) Spatial discontinuity changes the wavelength $\lambda$ but conserves the frequency $\omega$; (b) Temporal discontinuity changes the frequency $\omega$ but conserves the wavelength $\lambda . \ldots \ldots \ldots \ldots$

2.3 (a) Photonic crystal cavity used for a numerical study of AWC (top); spectrum of the light inside the cavity before and after the tuning (middle); temporal profile of the refractive index (bottom). (b) Energy change as function of time (top); shift of the wavelength $\Delta \lambda$ and change in energy $\Delta U$ as functions of $\Delta n$ (bottom). (After Ref. [1];C)2006 APS.) 18 
2.4 (a) Experimental setup of the first AWC demonstration with a silicon ring resonator.(b) Linearity of the AWC process. (c) Impact of cavity photon lifetime on AWC. (After Ref. [2]; C)2007 Nature Photonics.)

2.5 (a) Experimental setup of the first AWC demonstration in a photonic crystal waveguide.(b) Pump laser shift the eigenmodes of the waveguide. (c) Experiment result agrees with theoretical prediction quite well.(After Ref. [3];C2010 APS.) . . . . . . . . . . . . . . . .

2.6 Self phase modulation of an optical pulse: pulse shape remains constant, but develops symmetric spectral broadening. . . . . . . . . . . 31

2.7 Signature of self-steepening effect: pulse peak moves towards the trailing part and the spectral broadening becomes asymmetric. . . . . . . . 33

2.8 A fundamental soliton maintains its shape unchanged propagating inside a nonlinear dispersive medium. . . . . . . . . . . . .

2.9 Input (dotted-green) and output (solid-red) shape and spectrum for an optical Gaussian pulse $T_{0}=8$ fs (a), 40 fs (b), 200 fs (c) in a nondispersive Raman nonlinear medium. . . . . . . . . . . . . .

3.1 Schematic illustration of a linear system with input and output signal $x(t)$ and $y(t)$. The whole system is fully described by its impulse response function $h\left(t, t^{\prime}\right) \ldots \ldots \ldots \ldots$ 
3.2 Schematic illustration of an optical pulse propagation through a dynamic or nonlinear optical medium. The impact of dielectric medium on pulse propagation is to perform a mapping of all the temporal locations from $t^{\prime}$ to $t$, and to scaling of the amplitudes of the electric field

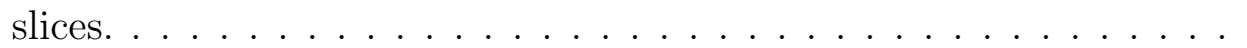

3.3 Electric slice mapping during a time transformation process. A input electric slice of height of $E_{\text {in }}\left(t^{\prime}\right)$, width of $d t^{\prime}$ is transferred to a corresponding output electric field slice of height of $E_{\text {out }}(t)$, width of $d t .50$

3.4 Temporal mapping in the cases of a static linear medium (blue-dotted), a dynamic linear medium (red-dashed), and a nonlinear Kerr medium (purple-solid). Depending on how refractive index changes with time, the temporal mapping can be linear or nonlinear. . . . . . . . . . . 52

4.1 Temporal profile for the refractive index in an AWC process: it changes from a stable value of $n_{1}$ beginning at $T_{i}$, following some arbitrary function, until it reaches to a new stable value $n_{2}$ after $T_{f} \quad \ldots \ldots 59$

4.2 Time transformation for a dynamic linear medium (solid-red) whose index decreases $\left(n_{1}>n_{2}\right)$. The case for a static linear medium (dottedblue) is also plotted for comparison. . . . . . . . . . . 
4.3 Input and output temporal powers, temporal phases, and spectral powers, spectral phases of a input Gaussian pulse for a AWC process. The dashed (blue), solid (green), and dotted (red) curves correspond to stretching factors of $s=3 / 2,1$, and $2 / 3$, respectively. . . . . . . .

4.4 Input and output electric fields (crosses) and their corresponding envelops (solid curve) associated with a four-cycle optical Gaussian pulse after propagating through a dynamic medium with $s=1.5 \ldots \ldots$. .

4.5 Four dynamic refractive index profiles and the corresponding output pulses. In all cases, the refractive index is initially $n_{1}$ and becomes $n_{2}$ after the change. The output pulses are identical in temporal shape and spectral power, but have different time delays. . . . . . . . . . . 68

4.6 Schematic illustration of reflection and transmission of an electromagnetic fields at a temporal boundary where the refractive index changes from $n_{1}$ (left panel) to its final value $n_{2}$ (right panel) at $t=t_{0} \ldots \ldots$

4.7 Electric field of a 4-cycle pulse after propagation across a temporal boundary. Reflected wave is generated because of impedance mismatch. The amplitudes of the reflected and transmitted fields agree with Eqs. (4.14) and (4.15). . . . . . . . . . . . . . 76 
4.8 Electric field of a 4-cycle pulse after propagation across a temporal boundary with impedance matching. No electric field is reflected back. The amplitudes of the reflected and transmitted fields agree with Eqs.

and $(4.15) \ldots \ldots \ldots \ldots \ldots \ldots$

4.9 Comparison of transmitted electric fields in cases 1 (blue curve, impedance changes) and 2 (yellow curve, impedance matched). Dotted black curve shows the analytic prediction based on Eq. (4.5). The input electric field is shown for comparison with a green curve. FDTD simulations confirm and agree fully with the predictions of the time transformation

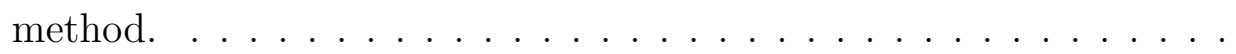

5.1 Schematic illustration of multiple round trips within a Fabry-Perot resonator. For a single input electric field slice, the output is a sequence of electric field slices with decreasing amplitude, due to the multibouncing of light by the two end mirrors. . . . . . . . . . . . .

5.2 Short-pulse (60 fs) propagation for an instantaneous change in refractive index of $-5 \%$ (blue) and $+5 \%$ (red) at time $T_{1}=250$ fs. The round-trip time is $233 \mathrm{fs}$. Detuning $\Delta \nu$ is defined as $\nu-\nu_{0}$, where $\nu_{0}$ is the input frequency. (a) The index change alters the width, amplitude, and delay of temporal pulses after $T_{c}$. The index change shifts the comb-like input spectrum (green curve) to higher $[+5 \%,(\mathrm{~b})]$ and lower $[-5 \%,(\mathrm{c})]$ frequencies at the FP output. . . . . . . . . 86 
5.3 Spectrogram of the output field in the case of $-5 \%$ index change at $T_{c}=250$ fs. A shift in central frequency of about $10 \mathrm{THz}$ is clearly seen after the first subpulse. . . . . . . . . . . . . . 88

5.4 Long-pulse (10 ps) propagation for a linear change in refractive index of $-0.1 \%$ (red) between $T_{i}=3 \mathrm{ps}$ and $T_{f}=6 \mathrm{ps}$ (marked by arrows). The round-trip time of the resonator is 0.23 ps. (a) The index change advances the output peak and produces a long tail with knee-like feature. (b) The output spectrum shows two spectral peaks corresponding to the original and shifted cavity modes, respectively. . . . . . . . .

5.5 Spectrogram for the situation shown in Fig. 5.4. The pulse spectrum appears to follow the cavity resonance indicated by the dotted line. .

5.6 Impact of index-change duration $\Delta T=T_{f}-T_{i}$ on propagation of 10-ps Gaussian pulses in the case of a $-0.1 \%$ linear change in the refractive index beginning at $T_{i}=3$ ps. (a) Output pulse narrows down and exhibits oscillations as $\Delta T$ decreases. (b) Amplitude of the AWC peak also increases as $\Delta T$ decreases. . . . . . . . . . . . . 95

5.7 AWC efficiency as a function of index-change duration $\Delta T$ for three values of photon lifetimes $\tau_{p h}$ in the case of $\Delta n=-0.1 \%$. The efficiency is maximum for $\Delta T$ near zero when $\tau_{p h}$ is relatively small but it peaks at a value of $\Delta T>0$ that increases with $\tau_{p h} \ldots \ldots \ldots$ 
5.8 Impact of the magnitude of $\Delta n$ for a fixed linear index-change duration of $3 \mathrm{ps}$, input pulse width of $10 \mathrm{ps}$, and a round-trip time of $0.23 \mathrm{ps.} \mathrm{(a)}$ The oscillation frequency in the pulse tail increases for a larger index change because of (b) a larger AWC-induced shift in the mode frequency. 98

5.9 Impact of detuning $\Delta \nu_{c}$ of input pulse from a cavity resonance $\left(\Delta \nu_{c}=\right.$ $\nu_{0}-\nu_{c}$, where $\nu_{0}$ and $\nu_{c}$ are the input carrier frequency and cavityresonance frequency, respectively) for a $-0.1 \%$ linear change in the refractive index between $T_{i}=3 \mathrm{ps}$ and $T_{f}=6 \mathrm{ps}$ (round-trip time is $0.23 \mathrm{ps}$ ). (a) Output pulse shapes show an asymmetry with respect to the the sign of $\Delta \nu_{c}$. (b) The amplitude of the AWC peak is larger for $\mathrm{a}+25 \mathrm{GHz}$ detuning than for the $-25 \mathrm{GHz}$ detuning (in this negative index-change case). . . . . . . . . . . . . . . . . . . . . . 100

5.10 Fraction of pulse energy transferred to the AWC peak as a function of detuning of input pulse from a cavity resonance under the same longpulse conditions as in Fig. 10 but for an index change of $-0.1 \%$ (blue)

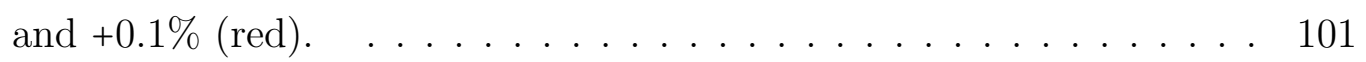

6.1 (a) Time transformation performed by a nonlinear Kerr medium for Gaussian pulse propagation and (b) the corresponding scaling factor s. Cases: $n_{2}>0$ (solid); $n_{2}<0$ (dashed); $n_{2}=0$ (dotted) $\ldots \ldots \ldots 106$ 
6.2 Output (dotted-black) and input (dashed-green) electric field for a single-cycle pulse after it propagates through a nonlinear Kerr medium. The predictions from FDTD calculation is also plotted for comparison using solid-yellow curve. Two approaches agree with each other quite well. . . . . . . . . . . . . . . . . . . . . 107

6.3 Output shape and spectrum of a 10-ps Gaussian pulse obtained with the time transformation method (solid lines) and using the NLS equation (dotted lines). Dashed curve shows input spectrum. . . . . . .

6.4 Schematic propagation of the electric field of an optical pulse through a Kerr medium $\left(n_{2}>0\right)$. Dotted curves show pulse envelope. Red time slices at the bottom move toward the back of the pulse.

6.5 Output shape and spectrum of a 100-fs Gaussian input pulse obtained with the time transformation method (solid lines) and using the NLS equation (dotted lines). Dashed curve shows input spectrum. . . . .

6.6 Shape (a) and spectrum (b) of a wide optical Gaussian pulse $\left(T_{0}=\right.$ $10 \mathrm{ps}$ ) at the input end (dashed green lines) and the output end at $z=4 L_{D}$ (dotted black lines). The predictions of the NLS equation are shown by solid yellow lines. . . . . . . . . . . . . . . . 116

6.7 The input (solid yellow) and output (dashed black) intensity profiles at $z=0$ and $z=10 L_{D}$ when parameters of the input pulse correspond to a fundamental soliton. . . . . . . . . . . . . . . . . . . 118 
6.8 Intensity profile of a sech pulse at a distance of $z=10 L_{D}$ for parameters identical to those of Fig. 6.7 except for $T_{0}=10 \mathrm{fs}$, which results in a self-steepening factor $1 /(4 \pi)$. Input profile is depicted by the dashedgreen curve. Predictions of the GNLS equation are shown by the solid

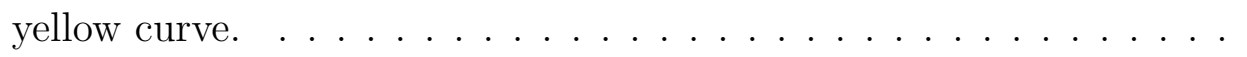

6.9 Evolution of the temporal and spectral intensity for a 10 fs third-order soliton $(N=3)$ propagates for a distance of $z=1 L_{D}$. The strength of the third order dispersion is chosen so that the normalized third order dispersion coefficient $\beta_{3} /\left(6 T_{0}\left|\beta_{2}\right|\right)=0.02 \ldots \ldots \ldots \ldots$

6.10 Electric field of a single-cycle pulse (dotted black curve) at $z=L_{D}$ (a) and $z=5 L_{D}$ (b) for $\omega_{0} /(2 \pi)=200 \mathrm{THz}, T_{0}=1 \mathrm{fs}$, and $N=1$. Input profile is depicted by the dashed green line. Predictions of the FDTD method are shown by the solid yellow line. . . . . . . . . . .

7.1 Evolution of temporal and spectral profiles over three dispersion lengths when an input pulse with $T_{0}=30$ fs forms a third-order soliton. The intensities are plotted using a normalized 50-dB color scale. . . . . . .

7.2 Pulse shape and spectrum of the 30 fs third-order soliton in Fig. 7.1 at $z=3 L_{D}$. Predictions of the time transformation method (solid-yellow) agree quite well with those from generalized NLS equation (dotted-

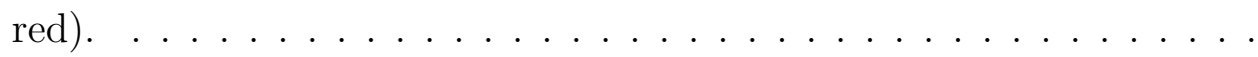


7.3 Comparison of pulse shapes (a) and spectra (b) at $z=3 L_{D}$ as $T_{0}$ is reduced from 20 to 1 fs. The input pulse corresponds to a third-order soliton in all cases. . . . . . . . . . . . . . . . . .

7.4 Changes in the Raman-induced frequency shift as $T_{0}$ is reduced from 20 to 1 fs for a fixed soliton order $N$. For the same value of $T_{0}$, pulses with a higher soliton order have larger frequency shifts. . . . . . . . . .

7.5 Energy of the dispersive wave as a function of $T_{0}$ for $N=2,3$, and 4 . The drop for $T_{0}<2$ fs is related to the development of rapid temporal

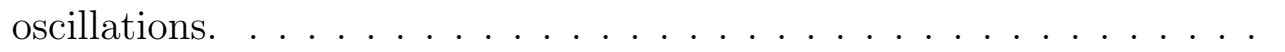

7.6 (a) Electric field and (b) temporal intensity at a distance of $3 L_{D}$ when a third-order soliton is excited using input pulse with $T_{0}=1 \mathrm{fs}$. The input electric field and intensities are shown for comparison by dotted

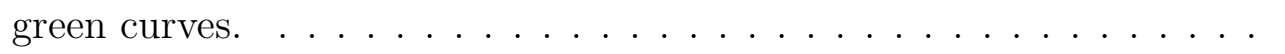

7.7 Spectrogram at $z=3 L_{D}$ for the $T_{0}=1$-fs case shown in Fig. 7.6. The intensity is plotted on a linear color scale. . . . . . . . . . . . . . . . 140

7.8 Impact of self-steepening on the shape (a) and spectrum (b) of a singlecycle input pulse. Self-steepening is turned off artificially for the dashed red curve but is included fully for the solid blue curve. The input curves are shown by dotted-green curves. . . . . . . . . . . . . . . . . 142 
8.1 (a) The electric fields and (b) optical spectra at the input (dashed blue curves) and output ends of a nondispersive Kerr medium using time transformation approach (dot-dashed red) and the FDTD method (solid yellow). . . . . . . . . . . . . . . . . . . . . 146

8.2 Changes in the relative amplitudes of the first three harmonics with the Kerr response time $\tau_{k}$. In all cases, the amplitude decreases almost exponentially with increasing $\tau_{k} . \ldots \ldots \ldots \ldots$. . . . . . 149

8.3 Electric fields of a $10 \mathrm{fs}$ optical pulse after it has propagated for 50 $\mu \mathrm{m}\left(4 L_{D}\right)$ and $150 \mu \mathrm{m}\left(12 L_{D}\right)$ in a linear (a) and nonlinear (b) dispersive medium. Nonlinearity balances the linear dispersion and helps

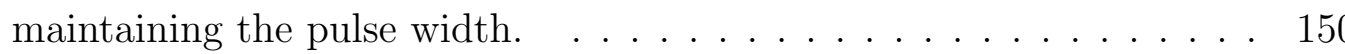

8.4 Comparison of time transformation (dashed red) and the FDTD (solid yellow) methods. Insert: expanded view of the "daughter" pulse oscillating at high frequencies. . . . . . . . . . . . . . . . . . . 152

A.1 Propagation of a multi-cycle soliton-like pulse over 10 dispersion lengths inside a Kerr medium whose dispersion is characterized by the Lorentz model. . . . . . . . . . . . . . . . . . . . . . . . . . . . 162 


\section{Introduction}

\subsection{Historical overview}

Throughout the human history, people have been trying to understand and use light, because it is closely related to our daily life. For example, sunshine is the source for all energy on earth, and artificial light is needed during nighttime. During the 18th century, different theories were proposed to describe light, among which the wave theory and the particle theory are the most famous. Now, scientists know that light can be viewed either as a "wave" described by the classical electromagnetic wave theory, or as a bunch of "photons" described by quantum theory. Although the understanding of light has increased quite a lot, people still put lots of effort to understand light itself as well as its interaction with different materials. One aspect of research effort is devoted to the study of propagation of short light pulses in a variety of optical media. Unlike the continuous wave $(\mathrm{CW})$ light, a light pulse only has a 
finite duration, as well as a time-dependent intensity profile. Such a unique feature makes light pulses very attractive, and it is very important for people to investigate and understand the propagation behavior of light pulses.

There are many motivations for us to understand light pulses, most of which are related either to an understanding of the underlying physics or for their applications. On the one hand, the propagation behavior of a light pulse inside various dielectric media itself is fascinating, and it is the foundation of many of our modern technologies. For example, the understanding of optical pulse propagation inside nonlinear dispersive media, such as silica glass, led to the development of modern fiber optical telecommunication systems, which are a core part of the Internet. Also, the appropriate interaction between nonlinearity and dispersion leads to the formation of solitons. Supercontinuum generation, which results from the interplay between a high-order soliton and a nonlinear dispersive medium has now been extensively used as a light source for medical imaging through a technique known as optical coherence tomography.

On the other hand, once we understand how a light pulse is affected by various factors during its propagation, it can provide us a perfect tool to probe dynamical processes that are unreachable by other means. For example, nowadays, people routinely use femtosecond laser pulses to investigate ultrafast chemical or biological processes, which happen within a picosecond or even shorter time scale. More recently, researchers are pushing into the atto-second region and developing techniques 
to generate, propagate and detect atto-second pulses. With the development of attosecond physics, we would be able to "see" some dynamic processes with atto-second resolution, which might give us a totally different picture of underlying physics, as well as new knowledge regarding our understanding of nature.

Not much happens when a light pulse propagates through a linear medium with a constant refractive index in time (a static medium). Except for a constant time-delay, neither the temporal shape nor the spectrum of the pulse change. However, lots of interesting phenomena happen if the medium becomes dynamic, in the sense that its refractive index changes with time (a dynamic linear medium) or in the sense that its refractive index changes with optical intensity (a nonlinear medium). This thesis is devoted to understanding the novel optical phenomena occurring in such dynamic linear and nonlinear optical media.

\subsection{Brief discussion of problems studied}

As discussed previously, understanding light pulse propagation behavior is important for its application, and also has a lot of interesting physics. In a dynamic linear medium, the frequency of light shifts, and the energy associated with the light changes as well. Directly solving Maxwell's equations using the finite difference time domain (FDTD) technique is able to give us the most accurate answer and capture all physics. However, this technique takes lot of time for computation and lacks the physical 
insight it can provide. On the other hand, a modal expansion approach correctly predicts the dynamic frequency shift without much computation burden. But this approach assumes discrete eigenmodes of the resonator or waveguide, which is not necessary true.

In a nonlinear medium, both pulse shape and spectrum change as a consequence of nonlinearity and dispersion. The nonlinear Schrödinger (NLS) equation is able to provide a very good description of pulses that are longer than few optical cycles. For ultrashort pulses that only contain a few optical cycles, the use of NLS equation becomes questionable because of the slowly varying envelope approximation (SVEA). With the development of ultrafast science, few-cycle even single-cycle pulses are available and they are attracting more and more research interests. In this region, people have to use FDTD to directly solve Maxwell's equation to study the nonlinear propagation of pulses, although FDTD is very time consuming and is limited by small propagation distance.

The objective of this thesis is to provide a novel method to study the propagation of optical pulses through dynamic media, including both dynamic linear media and nonlinear media. This method should be based directly on the electric field. This has two advantages: it would be able to apply to pulses with arbitrary durations because SVEA is not assumed; it can tell the phase information, which is more important than the envelope in some cases. The computation speed of this approach should be much faster than FDTD so that it could be used for long distance propagation. 


\subsection{Thesis outline}

The contents of this thesis are composed of three major parts: the first part describes the background material (Chapter 2) and introduces the time-transformation technique (Chapter 3); the second part applies this technique to dynamic linear media, both in the form of a traveling waveguide (Chapter 4) and an optical resonator (Chapter 5); the third part is devoted to its application to nonlinear media (Chapter 6-8). Specifically, this thesis is organized as follows.

Chapter 2 reviews the background of this thesis. Important effects such as optical pulses propagating through both dynamic linear and nonlinear media are described, and the past work on these topics is briefly summarized.

In Chapter 3, the time transformation method is proposed to study the propagation of optical pulses through dynamic media (including both dynamic linear and nonlinear media) whose indices change with time. This method is developed by extending the delta-function concept of impulse response from the linear system theory and incorporating a physical picture of the time delay by a medium. The impact of chromatic dispersion is also appropriately included so that this method can be applied to general optical media with arbitrary form of $n(t)$ and $n(\omega)$.

Chapter 4 applies this time transformation method to dynamic linear media. This new method correctly predicts the linear shift in light frequency, the change in the energy, and the conservation of the "adiabatic invariant" (number of photon) associated with the AWC process. Moreover, this method reveals other changes to the 
optical pulses that have not been noticed, such as pulse compression and spectral broadening. Most significantly, it is found that AWC alters the pulse power, chirp, and delay. The impact of details of how index is changed in time on a AWC process is investigated. The issue of reflection due to the index change is also discussed.

Chapter 5 extends the analysis in Chapter 4 to resonators, where the impact of different system parameters on a AWC process is discussed. It is shown that the AWC process in resonators results from a scaling of the round-trip time with index changes. More energy can be transferred to the new wavelength when the input pulses are slightly detuned from the cavity resonance and the refractive index does not change too rapidly. In fact, the optimum duration of index changes scales with the photon lifetime of the resonator.

Chapters 6 to 8 focus on nonlinear media. More specifically, Kerr nonlinear media are considered in Chapter 6. Conceptually, it is shown that the effect of propagation through a nonlinear medium is to change the relative spacing and duration of various temporal slices of the electric field associated with a light pulse. These temporal changes manifest as self-phase modulation in the spectral domain and self-steepening in the temporal domain. Chromatic dispersion is also included and the propagation of optical solitons is studied.

Chapter 7 extends the time-transformation technique by including the delayed Raman response and uses it to study propagation of ultrashort, few-cycle, optical pulses inside a nonlinear dispersive medium. It is found that for a fixed soliton order, 
the Raman-induced frequency shift becomes smaller, while the fraction of energy transferred to the dispersive wave increases, as pulse width is reduced. In the special case of a single-cycle pulse, the most dominant effect is self-steepening and it leads to dramatically new features in both the shape and spectrum of output pulses.

Chapter 8 considers the propagation of few-cycle optical pulses in a nonlinear Kerr medium considering the nonlinearity brought out by the real electric field $\mathbf{E}$, rather than the usually averaged value from the pulse envelop $\mathbf{A}$. The application of time transformation to such a nonlinear medium correctly predicts carrier-wave shocking and generation of odd-order harmonics inside a Kerr medium. The impact of a finite response of the Kerr nonlinearity on harmonic generation is investigated and chromatic dispersion is also included to study the soliton propagation.

As a concluding chapter, Chap. 9 summarizes this thesis. Final thoughts related to the future research of this thesis is also brought out as a personal perspective in this chapter.

The extension of the time transformation technique to pulse envelopes is discussed in Appendix A. It is shown that it can be easily applied to pulse envelopes and hence can be used conveniently to relatively long pulses. The computation efficiency and accuracy is studied in detail, and compared with those of the NLS equation. A MATLAB program is provided in Appendix B to show the numerical implementation of the time transformation method to a nonlinear dispersive medium. 


\section{Background and Review of Past}

\section{Work}

In this chapter, we briefly go through the background material for this thesis and review of the past work. The first part focuses on the frequency shift of light inside a dynamic linear medium, where a novel phenomenon of "adiabatic wavelength conversion" is described and past work on this topic is summarized. The second part of this chapter is devoted to a nonlinear medium, where some important effects occurring during pulse propagation are introduced.

\subsection{Dynamic linear media}

Let us first consider a dynamic linear medium. By calling a "dynamic linear" medium, the refractive index of the whole medium is assumed to change with time uniformly. Because of a time-dependent refractive index change, it is found that the spectrum of light shifts as a light pulse propagates through such a medium, and the term 
"adiabatic wavelength conversion" (AWC) is sometimes used to describe such dynamic frequency shift.

\subsubsection{Adiabatic wavelength conversion}

It is known that the spectrum of light does not change during its propagation inside a linear medium of temporally constant refractive index [4]. However, it was observed recently that light wavelength can change inside a dynamic linear medium whose refractive index changes with time [2-51]. The AWC process shown in Fig. 2.1 can be thought of three steps. Initially, an input optical pulse with wavelength $\lambda_{1}$ is resonantly coupled into a resonator. An optical mode at $\lambda_{1}$ would oscillate inside the resonator as a result of the resonant injection. When the optical pulse is oscillating within the resonator, the cavity resonance is dynamically tuned, which can be achieved by changing its refractive index through external means, such as the electrooptic effect (injection of free charge carriers in a semiconductor, the Pockels effect, etc.) or a purely optical effect (free carrier generation through optical absorption, cross-phase modulation, etc.). As a result of this cavity tuning, light inside the cavity shifts its wavelength accordingly to the new resonant wavelength $\lambda_{2}$ of the tuned cavity. There are two important facts about AWC. Firstly, its happening needs a temporal change of the refractive index within the photon lifetime of the cavity so that light inside the cavity can "see" the tuning process. Secondly, the amount of wavelength shift is linearly proportional to amount of index change, and this wavelength 


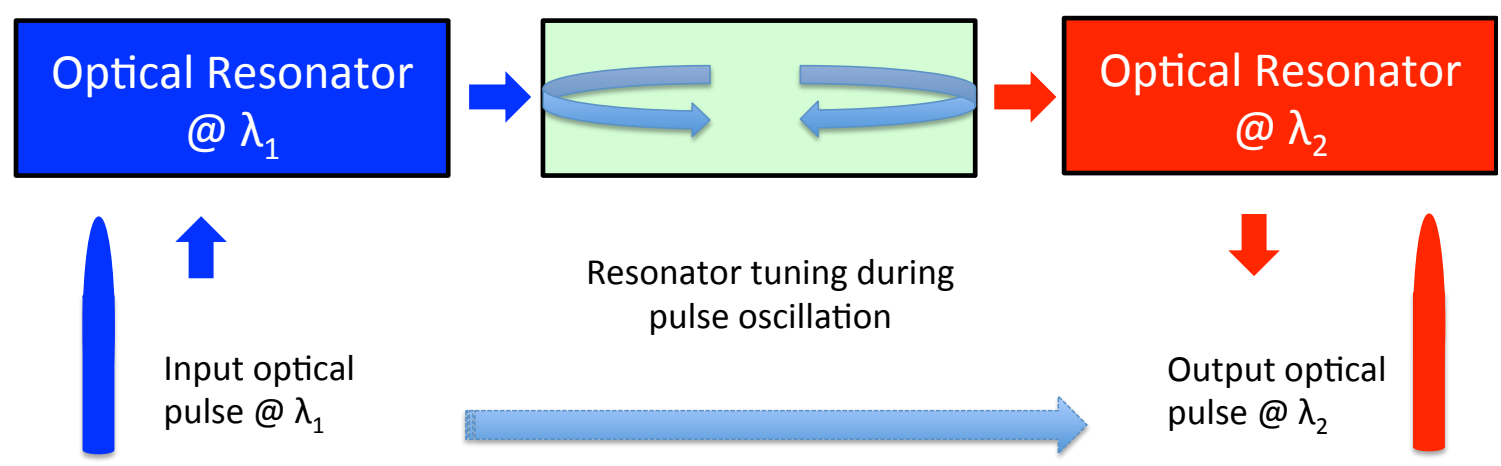

Figure 2.1: Schematic illustration of the AWC process. AWC happens because of a time-dependent refractive index of the medium inside a resonator, and the shift in wavelength is linear proportional to change in the refractive index.

shift does not depend on details of how the refractive index is changed.

As the refractive index is changed, energy exchange also happens between light and the medium. Unlike other ways of wavelength conversion, where the total energy is conserved, the conserved quantity associated with the AWC process is the number of photons, which is just the ratio of the total energy to the optical frequency: $U / \omega$. The number of photons is conserved while the refractive index is changed, which is similar to an adiabatic process where an adiabatic invariant remains constant, a wellknown concept in classical mechanics [5]. This is the reason why this process is called "adiabatic wavelength conversion." We can understand the AWC picture by thinking of an optical resonator as a musical instrument, such as a guitar. A mode of light oscillating inside the resonator is like a vibrating string of a guitar, both having a resonance frequency. We can generate a note in a guitar by plucking its string, and then raise up or lower down its frequency by tuning the string before vibration dies 
out. A similar thing happens for an oscillating mode of light inside a resonator, the only difference is that we tune a guitar by changing tension of a guitar string, while we tune an optical resonator by changing the refractive index of the medium inside it.

One can have a better understanding of AWC by considering the propagation of an electromagnetic wave through temporal or spatial boundaries. The case of a spatial boundary is well-known and Fresnel equations provide the transmission and reflection coefficients. Let us first consider this simple scenario shown in Fig. 2.2(a): a monochromatic plane wave propagates from medium 1 with refractive index $n_{1}$ into medium 2 with refractive index $n_{2}$, both of which are linear and lossless. From the boundary condition that the tangential part of the electric field is continuous, frequency of light $\omega$ cannot change. However, the wave number $k=n \omega / c$ is not a conserved quantity because of a spatial discontinuity in refractive index $\left(n_{2} \neq n_{1}\right)$. Actually, one can calculate this momentum exchange between the optical field and the medium when light travels from one dielectric medium into another medium by using the Lorentz force formula [6]. For the spatial boundary case, the electric field periodicity is not conserved spatially $\left(\lambda_{1} \neq \lambda_{2}\right)$, but is conserved temporally $\left(\omega_{1}=\omega_{2}\right)$.

Consider next a temporal discontinuity of the refractive index shown in Fig. 2.2(b): a monochromatic plane wave propagates in a time-varying medium. In this case, the medium initially has a constant refractive index $n_{1}$. The refractive index of the whole medium is changed instantaneously to a different value of $n_{2}$ at some time when light 


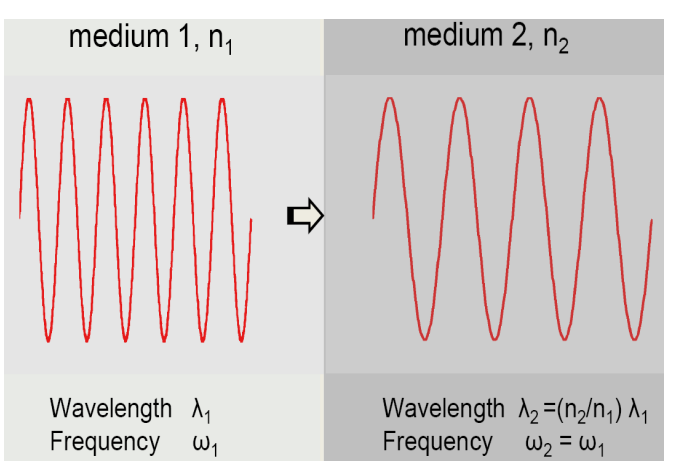

(a)
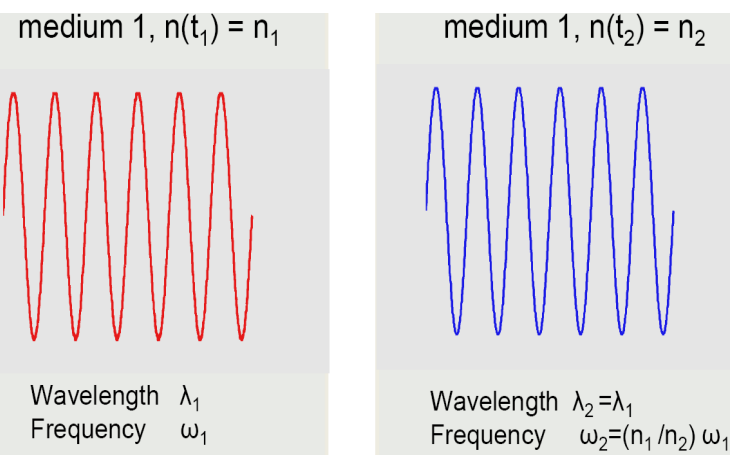

(b)

Figure 2.2: Comparison of spatial and temporal refractive index discontinuities.

(a) Spatial discontinuity changes the wavelength $\lambda$ but conserves the frequency

$\omega$; (b) Temporal discontinuity changes the frequency $\omega$ but conserves the wavelength $\lambda$.

is propagating inside the medium. Because light is fully contained inside the medium when the refractive index is being changed, the photon momentum or wave number $(k=n \omega / c)$ is conserved during this process [6]. To meet this conservation, light has to shift its frequency to the new value $\omega_{2}=\omega_{1} n_{1} / n_{2}$ as the refractive index is changed from $n_{1}$ to $n_{2}$. It is clear that the direction of change of frequency $\omega$ is inverse to that of refractive index $n$. In this case, energy is not a conserved quantity because this is an open system. Exchange of energy between light and the medium happens when the refractive index of the medium is changed. Therefore, at a temporal boundary, the electric field periodicity is not conserved temporally $\left(\omega_{1} \neq \omega_{2}\right)$, but is conserved spatially $\left(\lambda_{1}=\lambda_{2}\right)$. 


\subsubsection{Previous studies in the microwave region}

The investigation of the frequency changes in dynamic linear media began as early as 1958. By directly solving Maxwell's equations, Morgenthaler considered dynamics of the frequency, energy, and the momentum of a propagating electromagnetic wave within a time-varying dielectric medium [7]. He showed that the shift in instantaneous frequency is proportional to the amount of index change, and a reflected wave might also be generated due to a change of the medium's impedance with time. The temporal boundary value problem was considered by Fante in Ref. [8], where expressions for the electromagnetic fields transmitted into a time-varying medium for a plane wave incident upon both on a non-dispersive and dispersive dielectric half-space were obtained. Especially, solutions were obtained for the case when the medium was changed in a stepwise fashion, and also for the case when the medium varied slowly and continuously. The propagation of a wave inside a medium whose refractive index varies slowly in time and space (as compared to the temporal period and wavelength of the wave) was studied by Landauer and Peng in 1973 [9]. In this theoretical work, the medium was viewed as to be represented by a series of small and moving dielectric constant discontinuities with a uniform medium between adjacent moving steps.

Plasma provides a practical platform to study the interaction of an electromagnetic wave with a time-varying medium. The presence of electron-hole pairs effectively changes the refractive index of the plasma, and what is more favorable is that the density of such electron-hole pairs can be modulated in a dynamic fashion quite easily. 
For example, the plasma can be dynamically generated by the ionization of a medium using a strong pump laser. Alternatively, the electron density could be changed simply by modulating the injection current into a semiconductor crystal. Jiang presented a systematic study on the propagation and radiation of an electromagnetic wave from sinusoidally oscillating sources in a suddenly-created plasma. By applying the Laplace transform, expressions for the fields were derived that showed the modulation of the wave frequency due to the presence of plasma [10]. Ruiz et al. used transformation techniques to study electromagnetic wave propagating in dynamic media [11]. In this work, the temporal boundary was discussed and both spherical and plane wave characteristics were considered, and the change in the wave frequency and amplitude were calculated using the Laplace transform.

The reflection occurring due to temporal changes in the refractive index ("a temporal boundary") was considered in detail by AuYeung [12]. Specially, it was pointed out that the backward wave is a phase-conjugate replica of the incident field and the dependence of the phase-conjugate reflectivity on the refractive index change was also investigated. Kalluri followed with an investigation of the reflection of an electromagnetic wave from a suddenly created plasma half-space, where a temporal discontinuity, a spatial discontinuity and a dispersive medium were involved [13]. The effects of quickly creating a plasma around a monochromatic electromagnetic source wave, on time scales on the order of a cycle of the wave, were considered by Wilks et al. [14]. In addition to the frequency shift due to the change of the plasma density, 
they also showed generation of a time-independent magnetic field, where a substantial fraction of the initial magnetic field energy was sustained. The problem of waves propagating in a time varying plasma in the presence of an external magnetic field was considered by Kalluri in 1989 [15]. By solving Maxwell's equations together with the equation of motion of the charged particles (electrons), a characteristic equation for the light frequency $\omega$ was obtained, which showed that the original wave splits into three new waves with different frequencies.

Esarey et al. proposed a new technique to up-shift the frequency of a laser pulse that utilized the interaction of the laser pulse itself with a co-propagating relativistic ionization front [16]. The case of electromagnetic wave penetration and scattering from a material half-space having sinusoidally time-varying conductivity was analyzed by Harfoush et al. in 1991 [17]. A soluble quantum theory model for the interaction of an electromagnetic field with a time-dependent dielectric medium was given by Cirone et al., and the electromagnetic field and photon statistics were found [18]. In a theoretical work, Berezhiani et al. showed that the interaction of a laser pulse with a nonstationary semiconductor plasma can lead to a variety of interesting phenomena, including the controlled upshifting of the laser frequency, which could lead to the possibility of tunable lasers over a wide range of frequencies, and the trapping (nonpropagation) of a substantial part of the incident pulse [19]. The propagation of an electromagnetic wave along a planar wave-guiding structure containing time-varying plasma was considered by Bakunov et al. They found some quantities (adiabatic in- 
variants) that relate the energy of the wave and its frequency to be conserved during the process of the plasma density variation on condition that the time scale of the variation is much greater than the wave period (adiabatic approximation) [20,21].

Soon after these early theoretical works, experimental investigations on electromagnetic wave propagation in time-varying media were carried out. In 1990, the self-frequency up-conversion of microwave pulses were demonstrated by using two crossed microwaves to generate a time-dependent plasma in their intersection region inside a chamber, and the results were shown to agree with the previous theoretical predictions [22]. By ionizing azulene vapor contained in a resonant cavity using a laser pulse, Joshi et al. showed a 5\% frequency shift of a RF wave with greater than $10 \%$ efficiency [23]. In 1992, the ionization-induced frequency shift of laser pulses propagating within atmospheric-density noble gases was studied by Penetrante et al. [24]. In this study, the laser frequency shifts in different kinds of nobel gases were investigated, and the numerical simulations were found to provide qualitative explanations of most of the features observed experimentally. Frequency up-shift and conversion of a CW microwave into a frequency up-shifted and chirped periodic pulses were demonstrated experimentally by Kuo and Ren [25]. Yugami et al. showed that self-frequency shifting could be realized by propagating a source wave inside a waveguide filled with tetrakis-dimethyl-amino-ethylene gas in a 2002 experimental work [26]. The source wave changed the gas into a overdense plasma through ionizing, and a self-frequency shift of the source wave from $9 \mathrm{GHz}$ to $22 \mathrm{GHz}$ was observed. 
Semiconductor crystals also provide a good platform to realize such dynamic frequency shifts of light. An experimental work was performed by Geltner et al. in 2002 [27]. By ionizing a ZnSe semiconductor crystal with transverse propagating picosecond pulses, wavelength shifts of up to $1.6 \mathrm{~nm}$ of a $0.8-\mu \mathrm{m}$ picosecond laser pulse were observed. By utilizing the ionization and recombination fronts which were created by two-photon absorption inside a semiconductor (ZnSe) plasma, , both up- and down-shift of the laser frequency, were demonstrated by Avitzour et al. in 2005 [28]. They also presented a general technique to measure the rapid ionization and recombination processes. The frequency up-conversion in the terahertz $(\mathrm{THz})$ region (a frequency shift from 0.35 to $3.5 \mathrm{THz}$ ) was demonstrated recently by Nishida et al. by flash ionization in a ZnSe crystal with a Ti:sapphire laser pulse [29].

\subsubsection{Recent studies in the optical region}

It was almost half century after 1958 that the problem of wave propagation in timevarying media was investigated by the optics community. These studies originated from the discussion of light within dynamic photonic structures. Reed et al. studied the interaction of light with shock waves in photonic crystals [30,31]. The shock waves effectively introduce a dynamic refractive index, which leads to a frequency shift or the "reversed Doppler effect" of light traveling inside. Followed by these works, Yanik and Fan discussed manipulating light by a combination of linear optical elements: resonators coupled with waveguides. They showed that the time reversal 
of light and stopping light could be realized optically by a small amount of refractive index change of these linear optical elements [32,33].

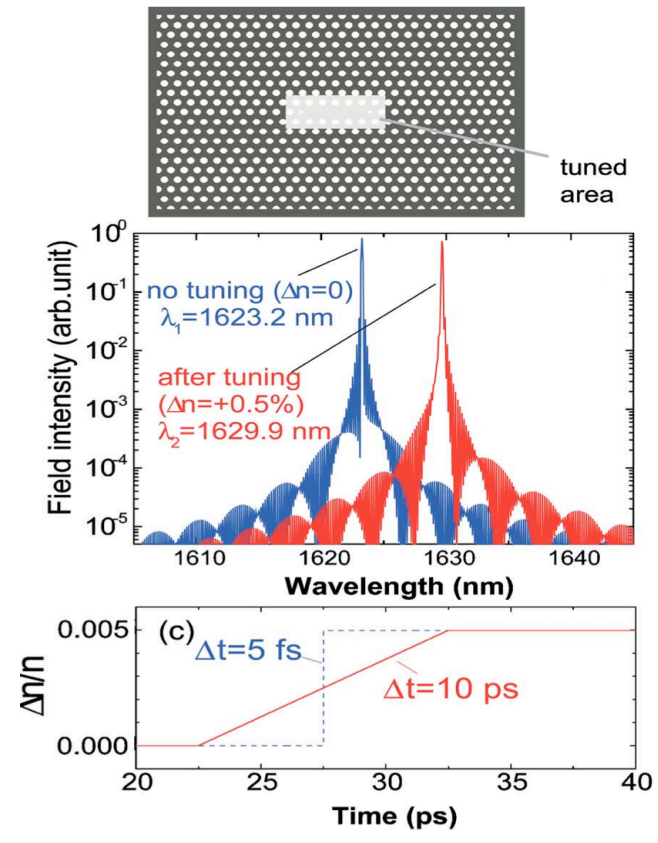

(a)
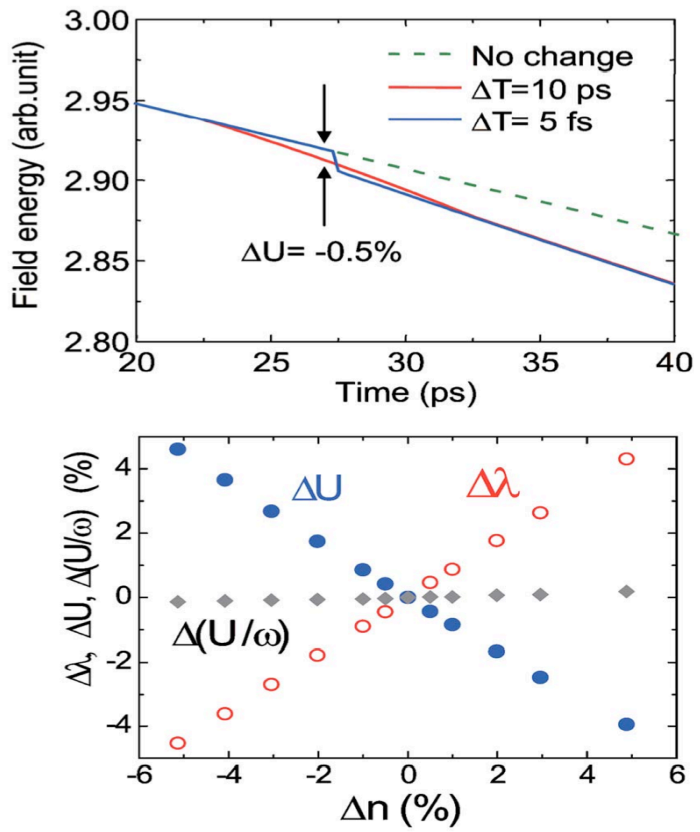

(b)

Figure 2.3: (a) Photonic crystal cavity used for a numerical study of AWC (top); spectrum of the light inside the cavity before and after the tuning (middle); temporal profile of the refractive index (bottom). (b) Energy change as function of time (top); shift of the wavelength $\Delta \lambda$ and change in energy $\Delta U$ as functions of $\Delta n$ (bottom). (After Ref. [1];(C)2006 APS.)

AWC was first discussed in a detailed fashion by Notomi and Mitsugi using the finite-difference time-domain (FDTD) numerical technique by directly solving Maxwell's equations for a photonic crystal cavity [1]. Figure 2.3 (a, top) shows the photonic crystal cavity (the gray area) for which they performed their numerical sim- 
ulations. In this study, they showed that the change in the wavelength inside the photonic crystal cavity was linearly proportional to the change in refractive index [Fig. 2.3 (a, central)] while the ratio of electromagnetic energy to the frequency of light, $U / \omega$, remained constant during the dynamic index change [Fig. 2.3(b, bottom)]. In addition, they showed that, unlike the conventional wavelength conversion process through nonlinear effects, AWC was a linear process and the shift in wavelength did not depend on details of how the refractive index was changed [Fig. 2.3(b, top)].

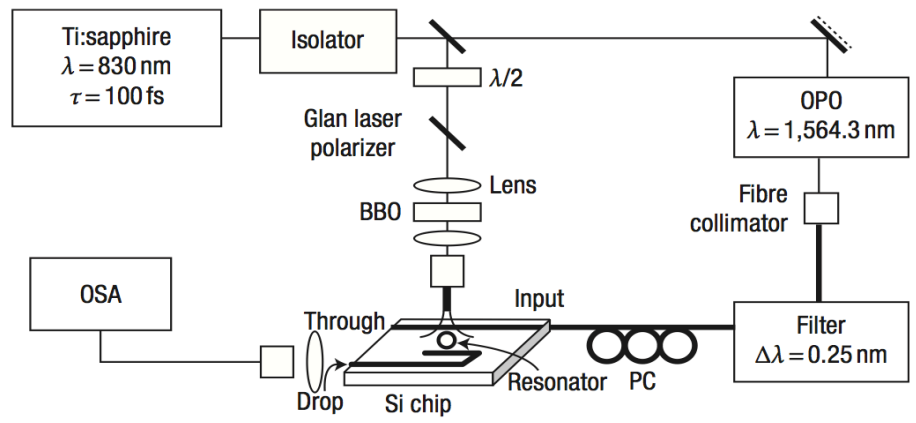

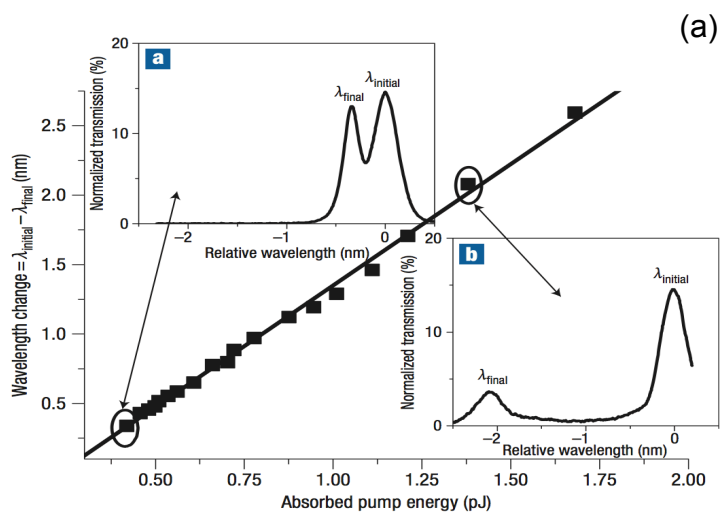

(b)

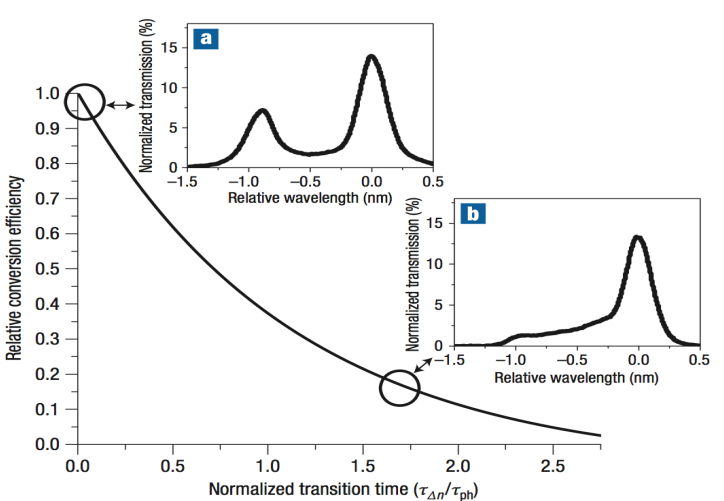

(c)

Figure 2.4: (a) Experimental setup of the first AWC demonstration with a silicon ring resonator.(b) Linearity of the AWC process. (c) Impact of cavity photon lifetime on AWC. (After Ref. [2]; C)2007 Nature Photonics.) 
The first experimental demonstration of AWC was performed by Preble et al. in 2007 [2]. As shown in Fig. 2.4 (a), the experiment was carried out in a silicon ring resonator in a pump-probe configuration. In order to change the refractive index of the cavity uniformly, a pump laser was shined on top of the cavity, a typical configuration that was used by most of the pump-probe AWC experiments. Figure 2.4 (b) shows the experimental confirmation of the linearity of AWC; experimental data match quite well the theoretical prediction. The impact of cavity photon lifetime is shown in Fig. 2.4 (c). In order to obtain a high conversion efficiency, refractive index changes had to happen well within the cavity photon lifetime, which is expected.

Since these two pioneer works, the AWC phenomenon has attracted considerable attention. In 2006, Gaburro et al. proposed using a time-dependent, spatially periodic photonic structure to shift the carrier frequency of an optical pulse that propagated within it [34]. By simulating ultrasmall double-layer photonic-crystal-slab cavities with high-Q values with a three dimensional FDTD technique, Notomi et al. showed a novel way of realizing AWC by mechanically tuning the cavity resonance [35]. Similar idea was proposed in a work by Khorshidahmad et al. in 2010 [36]. In 2007, McCutcheon et al. performed an ultrafast pump-probe experiment on wavelengthscale, silicon-based optical microcavities. [37]. By changing the delay time between the pump and probe, the emission spectrum of electromagnetic energy stored within a dynamic cavity was studied. The first experimental demonstration of AWC in a Fabry-Perot type photonic crystal cavity was performed by Tanabe et al. in 2009 [38]. 
Elshaari and Preble proposed a novel way to build an active optical isolator using adiabatic wavelength conversion in microcavities [39]. Sánchez et al. investigated the interaction of light with a dielectric slab with a time-periodic dielectric function $\epsilon(\mathrm{t})$. More specifically, the transmission and reflection coefficients were derived [40], and the pulse case was discussed in detail [41]. Recently, Daniel et al. found that Gires-Tournois resonators actually provided higher AWC efficiency [42]. AWC was experimentally demonstrated at a single-photon level using an integrated silicon ring resonator recently by Preble et al. [43].

Biancalana et al. presented a detailed theoretical study of the dynamics of light propagation in spatiotemporal dielectric structures [44]. Speficially, a two-component, highly symmetric version of Maxwell's equation was used to analyze the propagation, transmission and reflection properties of electromagnetic waves in one-dimensional non-dispersive dielectric media possessing arbitrary space-time dependence of the refractive index. Mendonsa et al. developed a concept of "time refraction" to describe the effects of a sudden temporal change of optical properties of a dielectric medium in 2000 [45], an elementary process associated with photon acceleration [46] and frequency upshift. Speficially, by extending the usual concepts of refraction and reflection into the time domain, the novel concepts of time refraction and time reflection were defined and characterized. The corresponding transmission and reflection coefficients for a "temporal boundary" were derived based on the appropriate boundary conditions [47]. 
In typical AWC studies, the refractive index of the cavity is assumed to change uniformly, and this is the case for most experiments. However, if one changes the refractive index only in a part of the medium, an interesting phenomenon happens due to this "imhomogeneous" tuning of the medium. By changing only a portion of the refractive index of the cavity over a time interval that was comparable to the inverse of the frequency separation of modes, Dong et al. demonstrated photonic transitions between discrete modes in a silicon optical microcavity [48]. Similar conclusion was reached in a theoretical paper by Daniel et al. [49]. This might be another potential application for AWC.

Earlier works on AWC focused on the wavelength shifts inside resonators. The reason why resonators are chosen is that they are able to trap light for a time (on the order of the cavity photon lifetime) long enough that the refractive index can be changed within this duration of time $[50,51]$. In fact, high-Q cavities with a photon lifetime of a few nanoseconds are available nowadays thanks to the development of modern fabrication techniques [52]. For traveling waveguides, this duration of time is their transit time $n L / c$, where $n, L$ are the refractive index and length of the waveguide and $c$ is the speed of light in vacuum. Even for a 1 -cm long silicon $(n \approx 3.5)$ waveguide, the transit time is only about $0.1 \mathrm{~ns}$. Also, in order to see the AWC effect, the whole pulse has to be fully contained in the medium when the index is changed. Considering all these facts, it is generally hard to realize AWC in a traveling waveguide. 
However, a resonator is not really required, and AWC can also happen in the traveling-wave configurations. The slow-light effect in photonic crystals makes AWC possible in a traveling waveguide. Using a modal approach, Kampfrath et al. predicted that frequency of a light pulse would shift if a waveguide was dynamically tuned when the pulse was propagating inside it [53]. Experimental confirmation occurred in 2010 by the same group using a slow-light photonic crystal waveguide [3]. Figure 2.5 (a) shows the configuration of this experiment. A pump laser was shined on the surface of the photonic crystal waveguide from the top when the probe pulse was fully contained inside. The pump light was absorbed by the waveguide and free carriers were consequently generated, leading to a modification of the refractive index of the medium. As shown in Fig. 2.5 (b), the pump laser shifts all eigenmodes of the waveguide and hence shifts the frequency of light inside it [Fig. 2.5 (c)]. In 2010, Upham et al. also proposed and experimentally demonstrated a novel way of realizing wavelength conversion by dynamically controlling the waveguide mode, which they called on-the-fly wavelength conversion [54].

\subsubsection{Different theoretical approaches}

Since the frequency shift of light in dynamic linear media was first discovered, many theoretical approaches have been used to investigate this interesting phenomenon. One way is to solve Maxwells' equations directly using the FDTD technique by imposing the time-dependent $\epsilon(t)$, and this approach was adopted by most early re- 


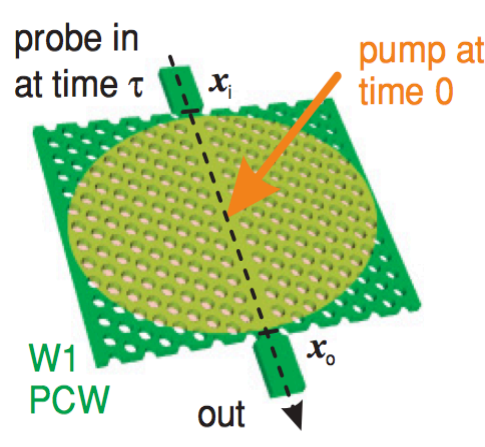

(a)

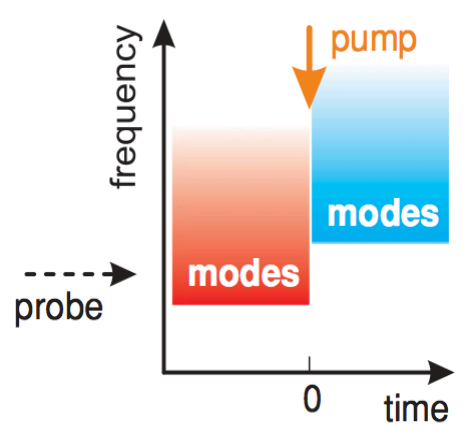

(b)

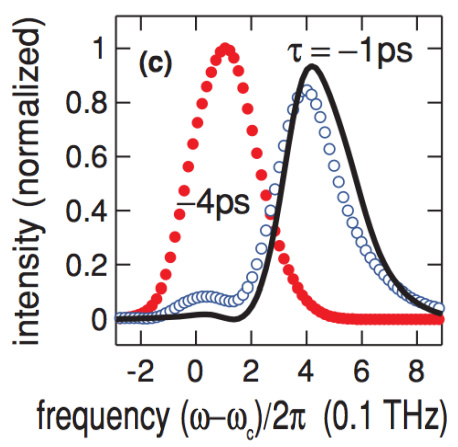

(c)

Figure 2.5: (a) Experimental setup of the first AWC demonstration in a photonic crystal waveguide.(b) Pump laser shift the eigenmodes of the waveguide. (c) Experiment result agrees with theoretical prediction quite well.(After Ref. [3];(C2010 APS.)

searchers $[1,30-33,35,38]$.

\section{Maxwell's equations}

Since light is essentially an electromagnetic wave, any study must start from Maxwell's equations, the master equations that fully characterize the behavior of electromagnetic fields. All of the dielectric media studied in this thesis are source-free, so we assume that both the free charge density $\rho$, and electric current density $\mathbf{J}$ to be zero. Maxwell's equations in this case can be expressed as

$$
\begin{gathered}
\nabla \cdot \mathbf{E}=0, \\
\nabla \cdot \mathbf{H}=0, \\
\nabla \times \mathbf{E}=-\frac{\partial \mathbf{B}}{\partial t},
\end{gathered}
$$




$$
\nabla \times \mathbf{H}=\frac{\partial \mathbf{D}}{\partial t}
$$

where $\mathbf{E}$ and $\mathbf{H}$ are the electric and magnetic fields, related to the electric displacement field $\mathbf{D}$ and the magnetic induction field $\mathbf{B}$ through the constitutive relations as

$$
\begin{aligned}
& \mathbf{B}=\mu_{0} \mathbf{H}+\mathbf{M}, \\
& \mathbf{D}=\epsilon_{0} \mathbf{E}+\mathbf{P},
\end{aligned}
$$

where $\epsilon_{0}$ and $\mu_{0}$ are the vacuum permittivity and permeability, respectively, $\mathbf{P}$ and $\mathbf{M}$ are the induced electric and magnetic polarizations. All characteristics for the medium are contained within $\mathbf{P}$ and $\mathbf{M}$. For a nonmagnetic medium $\mathbf{M}=0$, while the electric polarization $\mathbf{P}$ usually contains two parts, a linear part and a nonlinear part. Including only the Kerr-type nonlinearity, we can write it as

$$
\mathbf{P}(t)=\epsilon_{0} \int_{-\infty}^{t} \chi_{1}\left(t-t^{\prime}\right) \mathbf{E}\left(t^{\prime}\right) d t^{\prime}+\epsilon_{0} \chi_{3} \mathbf{E}^{3}\left(t^{\prime}\right) d t^{\prime}
$$

where the first term related to $\chi_{1}$ accounts for the linear response. The second term related to $\chi_{3}$ accounts for the nonlinear response, where we have assumed an instantaneous response of the Kerr nonlinearity. Equations (2.1-2.2) can be solved numerically, and this is the basic idea behind the finite-difference-time-domain (FDTD) algorithm.

\section{The Finite-difference-time-domain (FDTD) algorithm}

To solve Maxwell's equations with the FDTD algorithm, one replaces the derivatives with their finite-difference approximations. To illustrate this idea, we focus on the 
one-dimensional propagation problem, where the spatial effects are ignored in the plane-wave approximation. Note that such an approximation contains most of the physics for pulse propagation problem because light is confined spatially in most cases, using waveguides, and the spatial profile of light does not change during propagation.

If we further assume that the $\mathbf{E}$ and $\mathbf{H}$ fields are polarized in the $\mathbf{x}$ - and $\mathbf{y}$ directions, respectively, the first two Maxwell's equations [Eqs. (2.1a) and (2.1b)] are automatically satisfied, and the remaining two equations can be written as

$$
\begin{aligned}
& \frac{E(z+\delta z, t)-E(z-\delta z, t)}{2 \delta z}=-\frac{B(z, t+\delta t)-B(z, t-\delta t)}{2 \delta t} \\
& \frac{H(z+\delta z, t)-H(z-\delta z, t)}{2 \delta z}=\frac{D(z, t+\delta t)-D(z, t-\delta t)}{2 \delta t}
\end{aligned}
$$

where $\delta z$ and $\delta t$ are the step sizes in space and time chosen to discrete the electromagnetic field, and a central difference approximation is used. Because a direct solution of Maxwell's equations with the FDTD algorithm makes no approximation, it has been widely used for many applications. However, it has its downside as well. Because the electric and magnetic fields $\mathbf{E}$ and $\mathbf{H}$ oscillate rapidly both in space and time, in numerical implementation of FDTD, the spatial and temporal step sizes $\delta z$ and $\delta t$, have to be a small fraction of the wavelength and an optical cycle, respectively. Although straightforward and simple, the FDTD technique is very time consuming and is restricted to short-distance propagations. Also, it generally cannot provide much physical insight.

To address this issue, two major approaches have been developed. The first one starts from Maxwell's equations, and arrives at simpler differential equations 
by making reasonable approximations, such as one-dimensional propagation, and the separation of the spatial and temporal part of the fields. With this simplifications, one can "see" the physical picture behind AWC by solving the corresponding equations $[7,12,44,55]$. Later, a model expansion approach was developed starting from Maxwell's equations. But treating light as eigenmodes of a resonator or a waveguide, and the change in refractive index as a perturbation to the system, this approach successfully predicts the shift of light frequency and hence is widely used as well $[3,42,48,49]$.

\section{Differential amplitude equation approach}

For a non-dispersive dynamic linear medium, the constitutive relations are just:

$$
\begin{aligned}
& \mathbf{B}=\mu(t) \mathbf{H}, \\
& \mathbf{D}=\epsilon(t) \mathbf{E},
\end{aligned}
$$

with time-dependent $\epsilon(t)$ and $\mu(t)$. We consider plane-wave solutions, with $\mathbf{E}$ field polarized in the $\hat{y}$ direction and $\mathbf{H}$ filed polarized in the $\hat{x}$ direction (a TEM mode). In this case, light propagates in the $\hat{z}$ direction and the field components $E_{y}$ and $H_{x}$ are only functions of $z$ and $t$. Substituting Eqs. (2.5) into Eqs. (2.1) and taking the second order derivatives, we obtain the wave equations for a TEM mode as:

$$
\begin{aligned}
& \frac{\partial^{2} E_{y}}{\partial z^{2}}=\mu \epsilon \ddot{E}_{y}+(\dot{\mu} \epsilon+2 \mu \dot{\epsilon}) \dot{E}_{y}+(\mu \ddot{\epsilon}+\dot{\mu} \dot{\epsilon}) E_{y}, \\
& \frac{\partial^{2} H_{x}}{\partial z^{2}}=\mu \epsilon \ddot{H}_{x}+(\mu \dot{\epsilon}+2 \dot{\mu} \epsilon) \dot{H}_{x}+(\ddot{\mu} \epsilon+\dot{\mu} \dot{\epsilon}) H_{x},
\end{aligned}
$$


where $\dot{f}=\frac{\partial f}{\partial t}$. The solutions of Eqs. (2.6) may be expressed as a combination of a spatial part and a temporal part as

$$
\begin{aligned}
& E_{y}=\frac{W_{E}}{\epsilon \sqrt{\mu}}\left(A e^{i \beta z}+B e^{-i \beta z}\right), \\
& H_{x}=\frac{W_{H}}{\mu \sqrt{\epsilon}}\left(A e^{i \beta z}+B e^{-i \beta z}\right),
\end{aligned}
$$

where $\beta$ is the propagation constant and the temporal part $W_{E}$ and $W_{H}$ satisfy the following equations:

$$
\begin{aligned}
& \ddot{W}_{E}+\left[\frac{\beta^{2}}{\mu \epsilon}+\frac{1}{4}\left(\frac{\dot{\mu}}{\mu}\right)^{2}-\frac{1}{2}\left(\frac{\ddot{\mu}}{\mu}\right)\right] W_{E}=0, \\
& \ddot{W}_{H}+\left[\frac{\beta^{2}}{\mu \epsilon}+\frac{1}{4}\left(\frac{\dot{\epsilon}}{\epsilon}\right)^{2}-\frac{1}{2}\left(\frac{\ddot{\epsilon}}{\epsilon}\right)\right] W_{H}=0 .
\end{aligned}
$$

In general, no analytical solution for Eqs. (2.8) can be found for arbitrary $\epsilon(t)$ and $\mu(t)$. However, exact solutions exist under certain special cases, and the shift of light frequency can be found $[7,55]$.

\section{Modal expansion approach}

In this approach, the electric field is expanded with respect to the instantaneous eigenmodes of the photonic structure, such as a resonator or a waveguide. Mathematically, we have [56]:

$$
\psi(t)=\Sigma_{k} a_{k}(t) \psi_{k}^{t}
$$

where $\psi$ is the total electric field and $\psi_{k}^{t}$ is the $k_{t h}$ instantaneous eigenmode of the structure and the summation is over all eigenmodes: $k=1,2,3, \ldots$ This formulation 
was proposed by Xu et al. They showed that Maxwell's equations can be formulated as an operator form that describes the temporal evolution of an electromagnetic field in an abstract vector space in a manner analogous to the Schrödinger equation in quantum mechanics [56]. Under such a framework, if one starts from Maxwell's equations and takes the dynamic refractive index $n(t)$ into consideration, one can obtain an equation governing the dynamics of the $k_{t h}$ individual eigenmode $[3,49]$ :

$$
\frac{d a_{k}}{d t}=-i \omega_{k}^{t} a_{k}+\Sigma_{m} \Gamma_{k m} a_{m},
$$

where $a_{k}$ is the amplitude of the $k_{t h}$ mode and the eigenfrequency associated with this mode $\omega_{k}^{t}$ depends on time as well in dynamic cases. The second term on the right side describes the mode coupling between the $k_{t h}$ and $m_{t h}$ mode either in a resonator [49] or in a waveguide [3]. The coupling coefficients $\Gamma_{k m}$ are functions both of the amplitude of the $m_{t h}(m \neq k)$ mode $a_{m}$ and the dynamic refractive index $n(t)$. If the adiabatic criteria is satisfied, this term vanishes, and an adiabatic solution of Eq. (2.10) could be obtained as:

$$
a_{k}(t)=A_{k} \Theta(t-\tau) \exp \left(-i \int_{\tau}^{t} d s \omega_{k}^{s}\right),
$$

where $\Theta(t)$ is the Heaviside step function, considering the initial condition $\psi(t)=0$ if $t<\tau$, and $A_{k}$ is the initial mode amplitude. One can clearly see from Eq. (2.11) that the instantaneous frequency of light follows the eigenfrequency associated with the initial mode, which is time-dependent and is inversely proportional to the refractive index of the medium. This conclusion confirms the linear nature of such frequency 
shifts and agrees with the previous discussion. If, however, the adiabatic criterion is not satisfied, the coupling term would result in the excitation of other eigenmodes. This feature has been explored to realize the generation of a frequency comb in a dynamic resonator $[48,49]$.

\section{$2.2 \quad$ Nonlinear media}

For a nonlinear medium, its refractive index depends on the light intensity. Specially, a nonlinear medium becomes a dynamic medium for a light pulse because of the timedependent intensity profile of that light pulse. There is a lot of interesting physics as a light pulse propagates inside a nonlinear medium. Let us first have a look at the most encountered case: a Kerr nonlinear medium.

\subsubsection{Instantaneous Kerr nonlinearity}

The refractive index of a Kerr medium can be written as [57]

$$
n=n_{0}+n_{2} I,
$$

where $n_{0}$ is the linear refractive index, $n_{2}$ is the Kerr coefficient and $I$ is the light intensity. When an optical pulse with an intensity profile $I(t)$ propagates through such a Kerr medium for length $L$, the total phase it accumulates accordingly is

$$
\phi \equiv n L / c=n_{0} L \omega_{0} / c+n_{2} I(t) L \omega_{0} / c,
$$


where $c$ is the speed of light in vacuum and $\omega_{0}$ is the angular frequency of light. Besides the constant phase shift, the pulse experiences a time-dependent nonlinear phase shift that follows the pulse shape itself. In other words, an optical pulse modulates its own phase when passing through a nonlinear medium. This kind of phase modulation, known as self-phase modulation (SPM), was first discovered in 1967 and manifests as spectral broadening of optical pulses after they pass through a nonlinear Kerr medium [58]. Figure (2.6) plots the shape and spectrum for a Gaussian pulse with frequency $f_{0}=\omega_{0} / 2 \pi=200 \mathrm{THz}$, and $I(t)=I_{0} e^{-\left(t / T_{0}\right)^{2}}$ before and after propagating inside a Kerr nonlinear medium, with a maximum nonlinear phase shift $\phi_{\max }=n_{2} I_{0} L \omega_{0} / c=3 \pi[59]$ and relatively long pulse width $T_{0}=100$ ps. As shown in the figure, as a pulse propagates through a Kerr medium, its shape remains the same. But it experiences symmetric spectral broadening, accompanied by side bands.

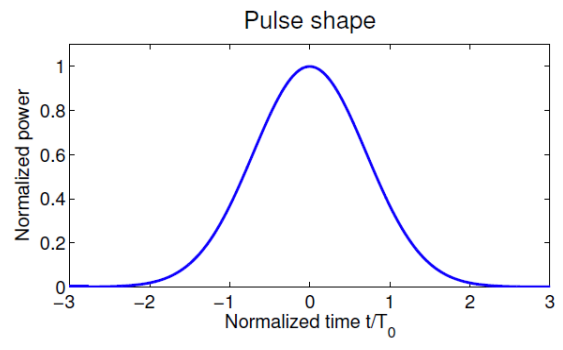

Pulse shape does not change.
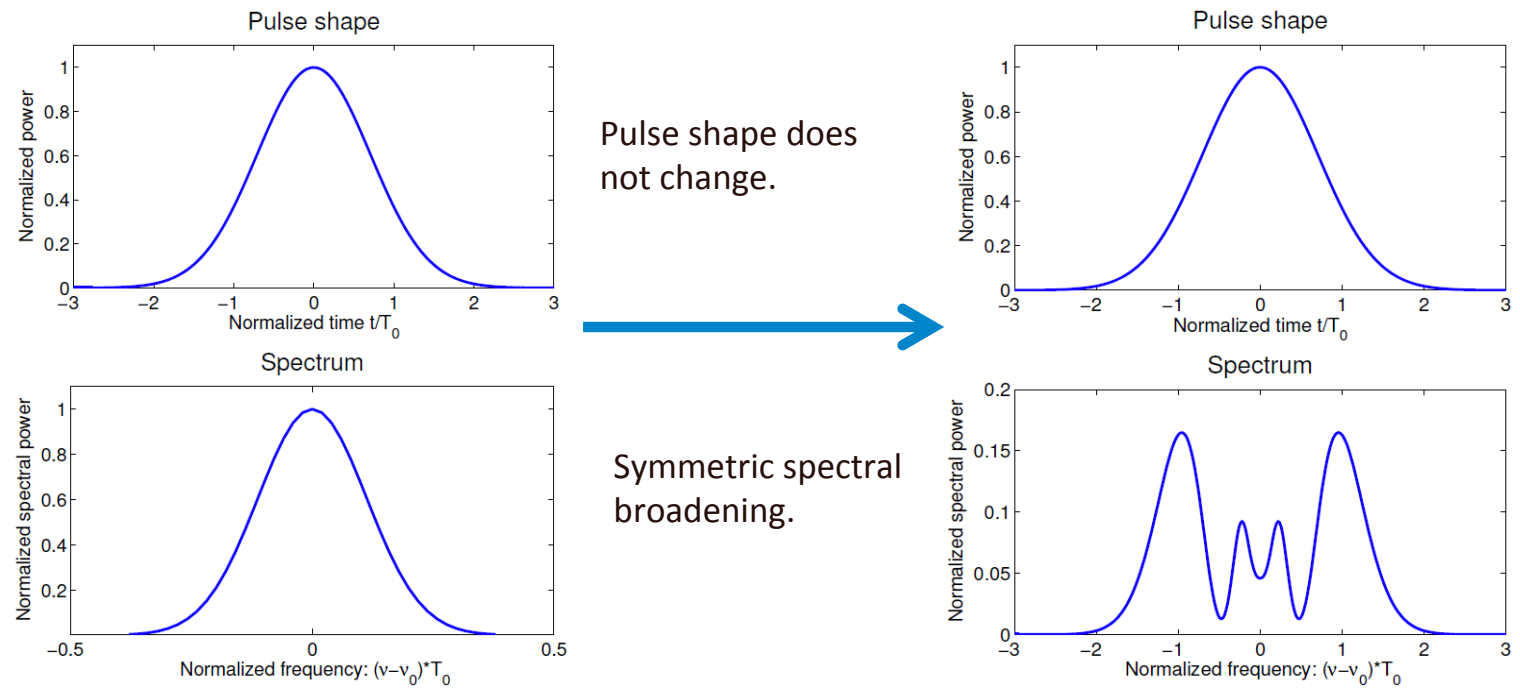

Figure 2.6: Self phase modulation of an optical pulse: pulse shape remains constant, but develops symmetric spectral broadening. 
When the width of an optical pulse is short enough, not only the phase is modulated by the pulse itself, its shape is also modulated as it propagates in a Kerr medium because of the intensity-dependent group velocity. This kind of pulse shape distortion is called self-steepening [60]. To evaluate the strength of self-steepening, one can define a self-steepening factor $1 /\left(\omega_{0} T_{0}\right)$ [59]. The effect of self-steepening becomes more obvious for larger value of the factor. Figure (2.7) plots the shape and spectrum for a short Gaussian pulse before and after propagating inside a Kerr nonlinear medium. All parameters are identical to those used in Fig. (2.6), except that the pulse width is reduced from 100 ps to 100 fs. Compared to the case shown in Fig. (2.6), pulse shape is distorted as its peak moves towards the trailing part, resulted from the selfsteepening effect. As a consequence of such pulse shape distortion, the symmetry in spectral broadening breaks down.

Mathematically, the behavior of a pulse propagation inside a nonlinear Kerr medium can be obtained by solving the following equation:

$$
\frac{\partial A}{\partial z}=i \gamma|A|^{2} A-\frac{\gamma}{\omega_{0}} \frac{\partial}{\partial T}\left(|A|^{2} A\right)
$$

where the pulse envelope $A(z, t)$ is related to the electric field of a light pulse having a propagation constant $\beta_{0} \equiv \beta\left(\omega_{0}\right)$ at its carrier frequency $\omega_{0}$ as

$$
E(z, t)=\operatorname{Re}\left[A\left(z, t-z / v_{g}\right) \exp \left(i \beta_{0} z-\omega_{0} t\right)\right]
$$

$T$ is measured in a frame of reference moving at the central group velocity of the pulse, and $\gamma=n_{2} \omega_{0} / c$ is a nonlinear coefficient. For the special case where the pulse 


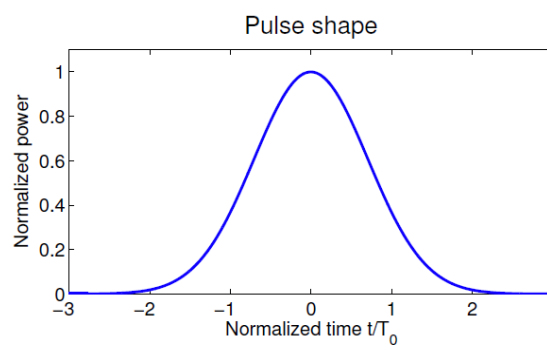

Peak moves towards the trailing part.
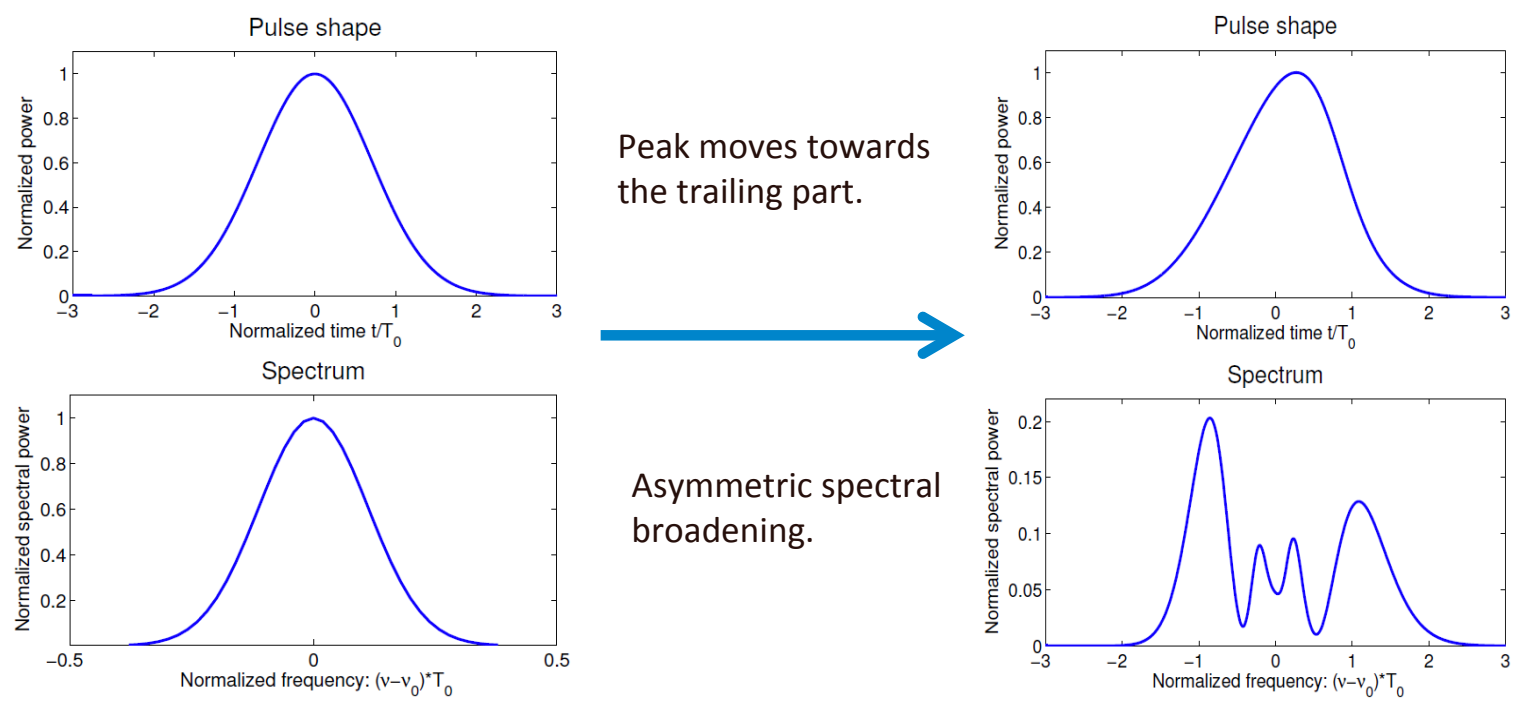

Figure 2.7: Signature of self-steepening effect: pulse peak moves towards the trailing part and the spectral broadening becomes asymmetric.

is relatively long and the second term in Eq. (2.14) can be neglected, one can directly write out the output field as:

$$
A(z, t)=A(0, t) \exp \left[i \gamma|A(0, t)|^{2} z\right] .
$$

As clearly seen from Eq. (2.16), the pulse remains its original input shape and the only effect of Kerr nonlinearity is to add a nonlinear phase shift to the pulse. As the nonlinear phase follows the pulse shape, the front part of the pulse experiences a blue shift while a red shift happens to the spectrum of the pulse tail.

In the presence of self-steepening, one cannot directly solve Eq. (2.14). However, an analytical solution of the pulse can be obtained by using the method of characteristics [61]. As the pulse propagate further, its peak shifts to the trailing part, and the self-steepening effect eventually leads to the formation of an optical shock [62]. 
In reality, optical shock does not usually occur because of the impact of dispersion. Before going though these effects, let's first have a look at the traditional approach to study propagation of optical pulses inside dispersive nonlinear media: The nonlinear Schrödinger equation.

\subsubsection{Nonlinear Schrödinger equation}

To solve a pulse propagation problem, one can always solve Maxwells equations with the FDTD technique. However, the FDTD approach is limited to relatively short propagation distances (typically $<1 \mathrm{~mm}$ ) because it requires a step size that is a small fraction of the optical wavelength. The most widely used approach for pulse propagation, especially in a dispersive nonlinear medium, is the nonlinear Schrödinger (NLS) equation, which is derived from Maxwells equations by making the slowly varying envelope approximation (SVEA). The NLS equation can be expressed in the following operator form [59]:

$$
\frac{\partial A}{\partial z}=(\hat{D}+\hat{N}) A
$$

where $\hat{D}$ and $\hat{N}$ are the differential and nonlinear operators, which contain the effects of dispersion and nonlinearity, and usually are expressed respectively as [59]

$$
\begin{gathered}
\hat{D}=-\frac{i \beta_{2}}{2} \frac{\partial^{2}}{\partial T^{2}}+\frac{\beta_{3}}{6} \frac{\partial^{3}}{\partial T^{3}}-\frac{\alpha}{2}, \\
\hat{N}=i \gamma\left(|A|^{2}+\frac{i}{\omega_{0}} \frac{1}{A} \frac{\partial}{\partial T}\left(|A|^{2} A\right)-T_{R} \frac{\partial|A|^{2}}{\partial T}\right),
\end{gathered}
$$


where $T=t-z / v_{g} \equiv t-\beta_{1} z$ is measured in a frame of reference moving at the group velocity of the pulse $v_{g}$ (retarded frame), $\alpha$ is the linear absorption coefficient, $\gamma$ is the nonlinear coefficient, $T_{R}$ is a parameter that depends on the Raman response function of the medium, which accounts for the non-instantaneous nonlinearity. Notice that linear absorption is included into the dispersion term, and the propagation constant is expanded as a Taylor series around the carrier frequency $\omega_{0}$ as [59]:

$$
\beta(\omega)=\beta_{0}+\left(\omega-\omega_{0}\right) \beta_{1}+\frac{1}{2}\left(\omega-\omega_{0}\right)^{2} \beta_{2}+\frac{1}{6}\left(\omega-\omega_{0}\right)^{3} \beta_{3}+\cdots
$$

where $\beta_{0} \equiv \beta\left(\omega_{0}\right)$, and the $m_{t h}$ order dispersion coefficients $\beta_{m}$ are defined as:

$$
\beta_{m}=\left(\frac{d^{m} \beta}{d \omega^{m}}\right)_{\omega=\omega_{0}} \quad(m=1,2, \ldots) .
$$

Equation (2.17) can be solved numerically with the split-step Fourier method [63]: nonlinearity and dispersion are taken care of in a step-wise fashion separately in the temporal and spectral domain, respectively.

Unlike the FDTD approach where the step size $\delta z$ has to be a small fraction of the light wavelength $\lambda$ (on the order of $\mu m$ ), the requirement on $\delta z$ in solving Eq. (2.17) is that it has to be a small fraction of the dispersion length $L_{D}=T_{0}^{2} /\left|\beta_{2}\right|\left(T_{0}\right.$ is the pulse width), since the pulse shape dose not change considerably within a dispersion length [59]. For a $T_{0}=1$ ps pulse propagating inside a typical optical fiber with $\beta_{2}=1 \mathrm{ps}^{2} / \mathrm{km}, L_{D}=1 \mathrm{~km}$. Therefore, the computation speed of NLS is much faster than FDTD. Though the use of NLS might become questionable for ultrashort pulses that only contain a few optical cycles due to SVEA, NLS is able to predict the pulse 
propagation behavior quite well in most situations.

\subsubsection{Impact of chromatic dispersion}

Essentially, all materials are dispersive, i.e. their refractive indices depend on the frequency of light. A direct consequence of dispersion is the frequency-dependent phase velocity $v_{p}=c / n(\omega)$ and group velocity $v_{g}=c /\left(n+\omega \frac{\partial n}{\partial \omega}\right)$. Sometimes dispersion is also called chromatic dispersion to emphasize its wavelength-dependent nature. The impact of dispersion cannot be neglected when the nonlinear propagation of optical pulses is considered, especially for ultrashort pulses having a very broadband spectrum [64]. The most well-known feature of the interplay of nonlinearity and dispersion is the fundamental optical soliton forming when the input pulse has a hyperbolic secant shape with an appropriate peak intensity so that the soliton condition is met [65].

Although the chromatic dispersion of a medium can be more accurately described by a Lorentz model [66], a Taylor expansion of the wave vector around the carrier frequency with dispersion coefficients $\beta_{i}, i=1,2,3 \ldots$ is simpler and more widely used [59]. For relatively long pulses, whose bandwidth is only a narrow fraction of the whole spectrum, it is enough to only consider the contribution from the first two terms in Eq. (2.19). The first term $\beta_{1}$ is related to the group velocity $v_{g}$ of the pulse, while $\beta_{2}$ is the second order dispersion coefficient, often called group-velocity dispersion (GVD). Self-steepening can also be neglected for long pulses. With all the 


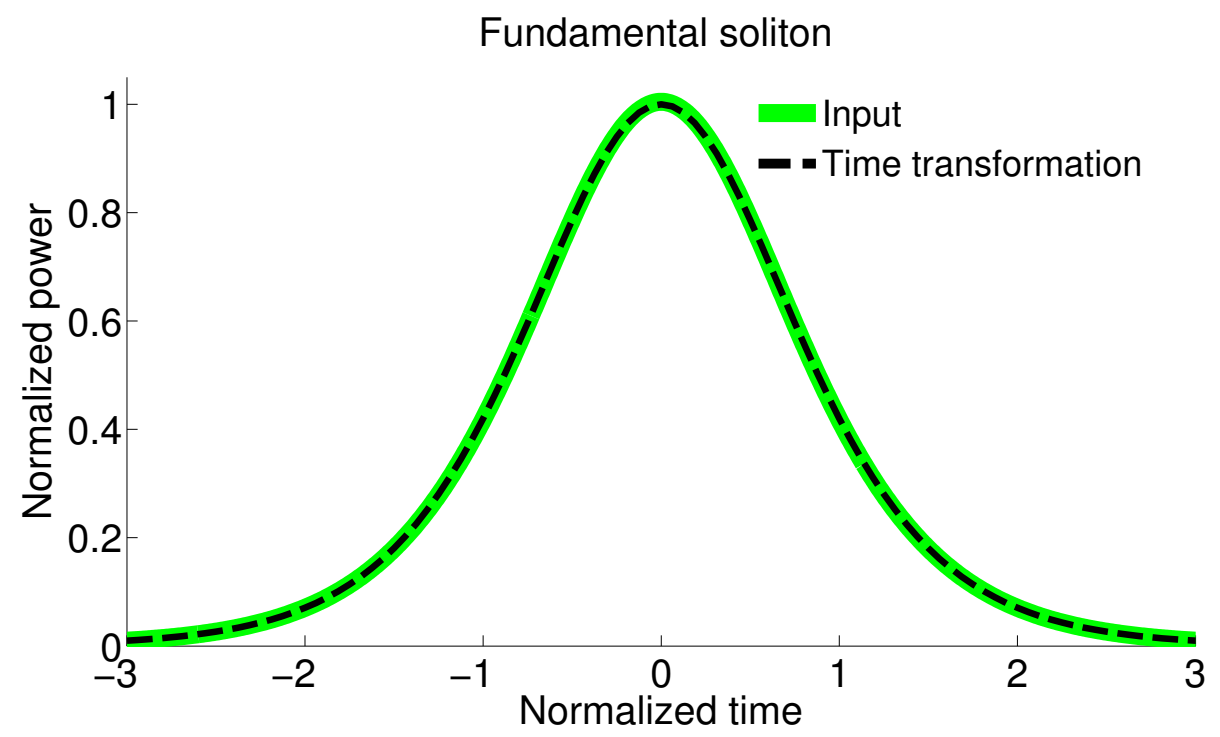

Figure 2.8: A fundamental soliton maintains its shape unchanged propagating inside a nonlinear dispersive medium.

assumptions, Eq. (2.17) is reduced to the so-called standard NLS equation:

$$
\frac{\partial A}{\partial z}=i \gamma|A|^{2} A+\frac{i \beta_{2}}{2} \frac{\partial^{2} A}{\partial T^{2}}
$$

where the first and second terms on the right side represent SPM and GVD, respectively.

Generally, there is no analytical solution for Eq. (2.21), and it needs to be solved numerically to obtain the output pulse for a specific input. One special case is the fundamental optical soliton, where the pulse has a hyperbolic-secant envelope:

$$
A_{\text {in }}(0, t)=\sqrt{P_{0}} \operatorname{sech}\left(t / T_{0}\right)
$$

where the choice of peak intensity $P_{0}$ and pulse width parameter $T_{0}$ is determined by 
the soliton condition [67]

$$
N^{2}=\frac{\gamma P_{0} T_{0}^{2}}{\left|\beta_{2}\right|}=1
$$

where $N$ is the soliton order. One can easily verify that the fundamental soliton indeed satisfies the standard NLS equation by directly substituting Eq. (2.22) into Eq. (2.21) and using Eq. (2.23). Figure (2.8) plots a fundamental soliton. The solidyellow curve shows the input pulse shape at $z=0$, while the dashed-black curve shows the output pulse shape at $z=10 L_{D}$ by solving the standard NLS equation Eq. (2.21). As seen in this figure, the output overlaps with the input perfectly, showing the soliton feature.

Solitons are very stable. A pulse would evolve into a soliton even when its input shape and intensity do not match the soliton condition. The stability of solitons is very helpful to fiber-optic communication systems. In order to transmit useful "information", the optical pulses has to remain their shapes within their own bit slots, without much distortion or broadening. It is very hard to maintain the pulse shape for long-distance propagation considering the impact of all kinds of nonlinear effects, dispersion, and other perturbations. The self-stable feature helps in solving this problem and, in fact, some fiber-optic communication systems use soliton-like pulses as the information carriers [67]. 


\subsubsection{Delayed Raman nonlinearity}

The nonlinear response of a medium generally has two parts. The first part comes from the response of electrons and is called the optical Kerr nonlinearity. The free electrons respond to the electric field associated with an optical pulse very quickly, occurring on a time scale of under $1 \mathrm{fs}$, and this is usually viewed as an instantaneous response. The other part is from the response of heavier molecules and is called the Raman nonlinearity. Molecules cannot respond to the electric field as fast as the electrons. For example, the response is delayed by about 60 fs in the case of silica fibers [68]. For relatively long pulses (1 ps or longer), the impact of delayed Raman nonlinearity can be neglected. However, when the pulse width becomes shorter than $100 \mathrm{fs}$, this delayed nonlinear response can no longer be neglected.

The impact of delayed Raman response can be incorporated into the NLS equation. If we ignore the instantaneous Kerr nonlinearity, the NLS equation for a nondispersive Raman medium becomes:

$$
\frac{\partial A(t, z)}{\partial z}=i \gamma A \int_{-\infty}^{t} R\left(t-t^{\prime}\right)\left|A\left(t^{\prime}\right)\right|^{2} d t^{\prime}-\frac{\gamma}{\omega_{0}} \frac{\partial}{\partial T}\left(A \int_{-\infty}^{t} R\left(t-t^{\prime}\right)\left|A\left(t^{\prime}\right)\right|^{2} d t^{\prime}\right),
$$

where $R(t)$ is the Raman response function. Note that by replacing $R(t)$ with the Kerr instantaneous response $\delta(t)$, Eq. (2.24) becomes Eq. (2.14), which is expected. Though a better description of the Raman response function for silica fibers was proposed by Lin and Agrawal [68], the classic Raman response function proposed by 

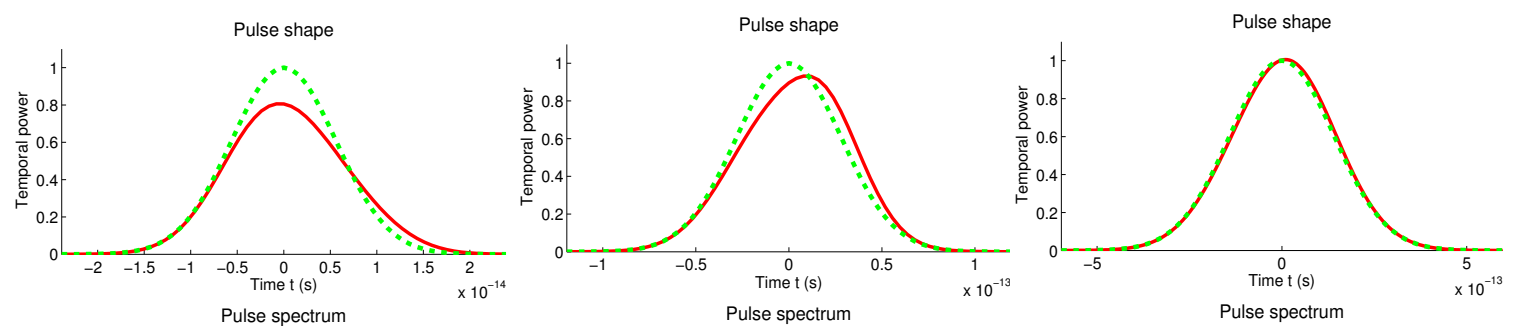

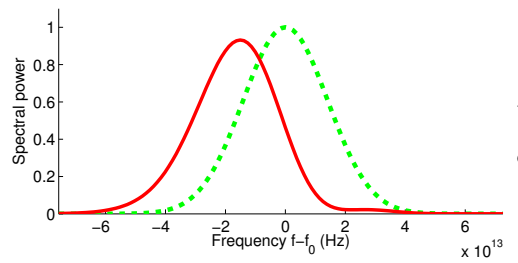

(a)

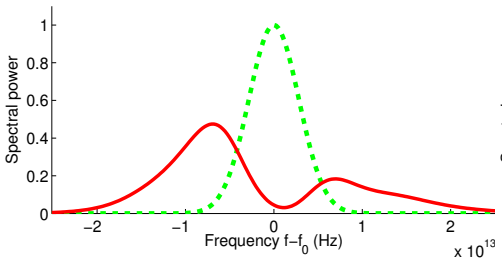

(b)

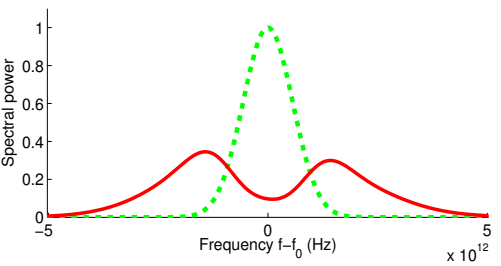

(c)

Figure 2.9: Input (dotted-green) and output (solid-red) shape and spectrum for an optical Gaussian pulse $T_{0}=8 \mathrm{fs}$ (a), $40 \mathrm{fs}$ (b), $200 \mathrm{fs}$ (c) in a non-dispersive Raman nonlinear medium.

Blow and Wood is often used in practice [69]:

$$
R(t)=\frac{\tau_{1}^{2}+\tau_{2}^{2}}{\tau_{1} \tau_{2}^{2}} \exp \left(-t / \tau_{2}\right) \sin \left(t / \tau_{1}\right)
$$

where $\tau_{1}=12.2$ fs is the phonon lifetime and $\tau_{2}=32$ fs is related to the bandwidth of the Lorentzian line.

To see the impact of Raman nonlinearity, we propagate three optical Gaussian pulses inside a Raman medium and plot the input and output shapes and spectra in Fig. (2.9). For these three pulses, we keep the same peak intensity, propagating length so that $\phi_{\max }=n_{2} I_{0} L \omega_{0} / c=1.5 \pi$ (with $f_{0}=\omega_{0} / 2 \pi=200 \mathrm{THz}$ ), but increase the pulse width $T_{0}$ from 8 fs (a) to 40 fs (b) and to 200 fs (c). As shown in Fig. 2.9 (a), spectrum of the 8-fs pulse experiences a red-shift, a signature of the delayed Raman nonlinearity. Such a frequency shift was first observed in 1986 on a soliton 
pulse, and was called the soliton self frequency shift [70], which was explained using the delayed Raman nonlinearity by Gordon [71]. As shown in Fig. 2.9 (b), when the pulse width $T_{0}$ increases from 8 fs to $40 \mathrm{fs}$, this frequency shift feature disappears, and the spectral changes exhibit features more like self-phase modulation. This behavior can be understood by noting that the Raman response has a characteristic time of $\tau_{2}=32$ fs and its impact becomes smaller as the pulse width gets larger. When the pulse is much wider than the characteristic response time, the delayed Raman response can be approximated as an instantaneous process. In fact, for the case $T_{0}=$ 200 fs shown in Fig. 2.9 (c), pulse shape remains constant, but develops symmetric spectral broadening, which is a SPM feature similar to that in Fig. (2.6). 


\section{Time Transformation Method}

This chapter presents a novel time transformation method for propagation of optical pulses in dynamic media. This method is developed based on the linear system theory, incorporating a conclusion of Maxwell's equations that the speed of light inside a medium is inversely proportional to its refractive index. The linear system theory would be reviewed first, and then how it can be applied to the propagation of a light pulse through an optical medium by connecting it to Maxwell's equations would be discussed. The time transformation method and its application to dynamic media are introduced in Section 3.3. The impact of dispersion is included into this method in Section 3.4. 


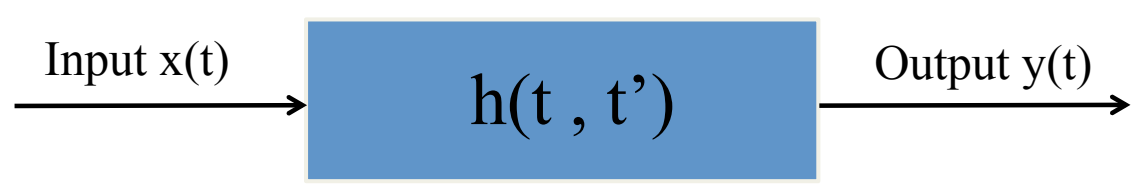

Figure 3.1: Schematic illustration of a linear system with input and output signal $x(t)$ and $y(t)$. The whole system is fully described by its impulse response function $h\left(t, t^{\prime}\right)$.

\subsection{Linear system theory}

Linear system theory has been used in a variety of fields, such as automatic control, signal processing, and telecommunications [72]. Although most of the real systems are fairly complex, many of them could be approximately viewed as "linear" under normal conditions and this can be described using the linear system theory.

Figure 3.1 illustrates the basic idea behind the linear system theory. As shown in this figure, the system is treated as a "black box" that processes an input signal $x(t)$, and outputs a corresponding signal $y(t)$ according to its impulse response function $h\left(t, t^{\prime}\right)$. Mathematically, a linear system could be fully characterized by its impulse response function $h\left(t, t^{\prime}\right)$ through the following integration [72]

$$
y(t)=\int_{-\infty}^{\infty} h\left(t, t^{\prime}\right) x\left(t^{\prime}\right) \mathrm{d} t^{\prime}
$$

where $x(t)$ and $y(t)$ are the input and output signal of the system, respectively. A system becomes time-invariant if the output does not depend on the particular time the input is applied. The general impulse response function $h\left(t, t^{\prime}\right)$ is reduced to a single variable function $h\left(t-t^{\prime}\right)$ for such linear time-invariant (LTI) systems. Thera are 
many unique features associated with a LTI system, among which the most important one is that a simple relation exists in the frequency domain:

$$
Y(\omega)=H(\omega) \tilde{X}(\omega)
$$

where $H(\omega)$ is the system's frequency response that is related to the impulse response function through the Fourier transform [73]

$$
H(\omega)=\int_{-\infty}^{\infty} h(t) e^{i \omega t} \mathrm{~d} t
$$

and $\tilde{X}(\omega)$ and $\tilde{Y}(\omega)$ are the spectrum of the input and output signals. Equation (3.2) could be obtained by taking the Fourier transform of both sides of Eq. (3.1), noticing that it becomes a convolution for a LTI system.

There are many implications of Eq. (3.2). The straightforward one is that a LTI system acts like a frequency filter, and no new frequency components can be generated. For a time-variant system, however, no such relation [Eq. (3.2)] exists in the frequency domain, and new frequency components can be generated.

A linear dielectric medium could be viewed as a linear system and pulse propagation through such a medium can be described by

$$
E_{\text {out }}(t)=\int_{-\infty}^{\infty} h\left(t, t^{\prime}\right) E_{\text {in }}\left(t^{\prime}\right) \mathrm{d} t^{\prime}
$$

where $E_{\text {in }}(t)$ and $E_{\text {out }}(t)$ are the input and output electric fields associated with the optical pulse. The key here is to find the impulse response function for a dielectric medium. 


\subsection{Solution of Maxwell's equations}

To find the impulse response function of a dielectric medium, we can start from Maxwell's equations. As discussed previously, there is no analytical solution for Maxwell's equations for a dielectric medium with arbitrary forms of permittivity $\epsilon$ and permeability $\mu$. However, for some special cases, Maxwell's equations can be solved exactly and analytical solutions can be obtained. One of these cases, and the most simple one is a static linear medium with constant $\epsilon=\epsilon_{0} \epsilon_{1}$ and $\mu=\mu_{0} \mu_{1}$, where $\epsilon_{1}$ and $\mu_{1}$ are the relative permittivity and permeability, respectively.

For such a medium, the constitutive relations become

$$
\begin{aligned}
& \mathbf{B}=\mu_{0} \mu_{1} \mathbf{H}, \\
& \mathbf{D}=\epsilon_{0} \epsilon_{1} \mathbf{E} .
\end{aligned}
$$

If we take the curl of Eq. (2.1c) and use Eqs. (2.1d) and (3.5), we obtain a secondorder differential equation that only contains the electric field $\mathbf{E}$ :

$$
\nabla \times \nabla \times \mathbf{E}=-\frac{n_{0}^{2}}{c^{2}} \frac{\partial^{2} \mathbf{E}}{\partial t^{2}}
$$

where $c=1 / \sqrt{\epsilon_{0} \mu_{0}}$ is the speed of light in vacuum and $n_{0}=\sqrt{\epsilon_{1} \mu_{1}}$ is the refractive index of the medium. Equation (3.6) can be further simplified using

$$
\nabla \times \nabla \times \mathbf{E} \equiv \nabla(\nabla \cdot \mathbf{E})-\nabla^{2} \mathbf{E}=-\nabla^{2} \mathbf{E}
$$


where Eq. (2.1a) is used. Substituting Eq. (3.7) into Eq. (3.6), we obtain the wave equation

$$
\nabla^{2} \mathbf{E}-\frac{n_{0}^{2}}{c^{2}} \frac{\partial^{2} \mathbf{E}}{\partial t^{2}}=0
$$

If we assume light propagation inside a single-mode optical waveguide, for which the spatial dependence of the waveguide mode does not change with propagation, Eq. (3.8) can be further simplified as

$$
\frac{\partial^{2} \mathbf{E}}{\partial z^{2}}-\frac{n_{0}^{2}}{c^{2}} \frac{\partial^{2} \mathbf{E}}{\partial t^{2}}=0
$$

whose solution in the special case of one dimensional propagation (in the $z$ direction) can be expressed as:

$$
E(z, t)=f\left(t-n_{0} z / c\right)+g\left(t+n_{0} z / c\right)
$$

where $f$ and $g$ represent a forward and backward traveling components with a propagation speed of $c / n_{0}$ and $-c / n_{0}$, respectively. Note that here we assume electric field is initially linearly polarized and we write a scaler field $E$ for simplicity. The exact forms of $f$ and $g$ need be determined through the initial condition.

Based on the above analysis, when a light pulse incidents into a medium with refractive index $n_{0}$, it would propagate inside such a medium at a velocity $c / n_{0}$. Consequently, for a medium of length $L$ with an input electric field at its input end $z=0$ propagating at the positive $z$ direction as

$$
E_{\text {in }}(t)=f(t)
$$


the output field at its output end is

$$
E_{\text {out }}(t)=f\left(t-n_{0} L / c\right)=E_{\text {in }}\left(t-n_{0} L / c\right),
$$

where we have ignored backward reflection of the input pulse. Recall the linear system theory in Eq. (3.4), the impulse response function for a linear dielectric medium is simply

$$
h\left(t-t^{\prime}\right)=\delta\left(t-t^{\prime}-T_{d 0}\right)
$$

where the delay time $T_{d 0}=n_{0} L / c$. One can have a better understanding of Eq. (3.13) by examining its Fourier transform pair in the frequency domain:

$$
H(\omega)=e^{i n_{0} \omega L / c}
$$

Eq. (3.14) tells us that only a constant phase $n_{0} \omega L / c$ is added to a plane wave at frequency $\omega$ when it propagates through a medium with refractive index $n_{0}$. Notice that the wave vector is $\beta=n_{0} \omega / c$ for a non-dispersive medium. Therefore, the constant phase is just $\phi=\beta L$, which meets our expectation.

\subsection{Time transformation method}

Linear system theory has been successfully applied to study pulse propagation in dispersive media [74]. Some of these studies focus on linear dispersive media. Here we extend this idea by incorporating a physical picture of the time delay by a medium, and propose a time transformation method to study pulse propagation. 
The basic idea behind the time transformation method can be understood from Fig. 3.2 where the propagation of an optical pulse through a dynamic optical medium whose refractive index $n(t)$ may change with time along its entire length $L$ is shown. More specifically, the input pulse is divided into a large number of temporal slices and a specific slice of duration $d t^{\prime}$ at input time $t^{\prime}$ (measured from an arbitrary origin $t=0)$ is focused on. The dielectric medium shift this electric field slice in the temporal domain with a transit-time delay $T_{d}\left(t^{\prime}\right)$ so that the output time of the slice is given by

$$
t=t^{\prime}+T_{d}\left(t^{\prime}\right)
$$

The electric field $E(t)$ associated with the input pulse changes during propagation and these changes affect both the temporal shape and the optical spectrum associated with the pulse. One can think of these changes as a simple consequence of the temporal shift of the electric slices from the input to the output, or a transformation of the temporal coordinate from $t^{\prime}$ to $t$. This is essentially a temporal mapping that maps the input slices to output slices. The key is to find the transit delay time $T_{d}\left(t^{\prime}\right)$ in the time transformation Eq. (3.15) associated with temporal mapping.

As is discussed in the previous section, the electric field slices travel at the same speed of $v_{0}=c / n_{0}$ for a very special case of a static linear medium whose refractive index is a constant $n_{0}$. It is straightforward to see that the transit time for all slices are the same and has a value of $T_{d}=n_{0} L / c$. The new feature of the time transformation in Eq. (3.15) is that the delay time $T_{d}$ is allowed to depend on the 


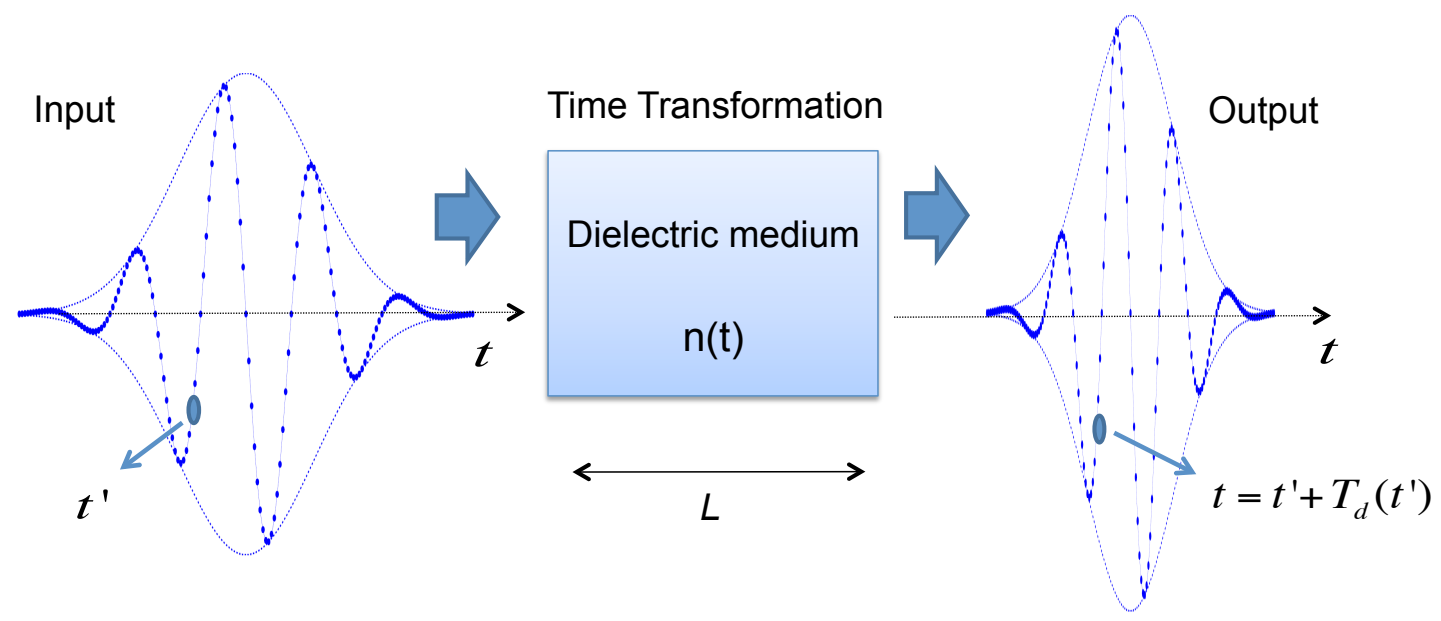

Figure 3.2: Schematic illustration of an optical pulse propagation through a dynamic or nonlinear optical medium. The impact of dielectric medium on pulse propagation is to perform a mapping of all the temporal locations from $t^{\prime}$ to $t$, and to scaling of the amplitudes of the electric field slices.

slice input time $t^{\prime}$. This dependence is clearly required to account for changes in the speed of light, $v(t)=c / n(t)$, occurring inside the medium in response to temporal changes in refractive index $n(t)$. In such dynamic case, this delay time $T_{d}$ can be determined through the following relation:

$$
\int_{t^{\prime}}^{t^{\prime}+T_{d}\left(t^{\prime}\right)}[c / n(t)] d t=L
$$

where $L$ is the length of the medium. Note that an implicit assumption is made here: the speed of light of a dynamic medium follows the instant refractive index of the medium as $v(t)=c / n(t)$. The validity of this assumption is confirmed in the next chapter by comparing the results from time transformation method with the direct solution of Maxwell's equations using FDTD. 


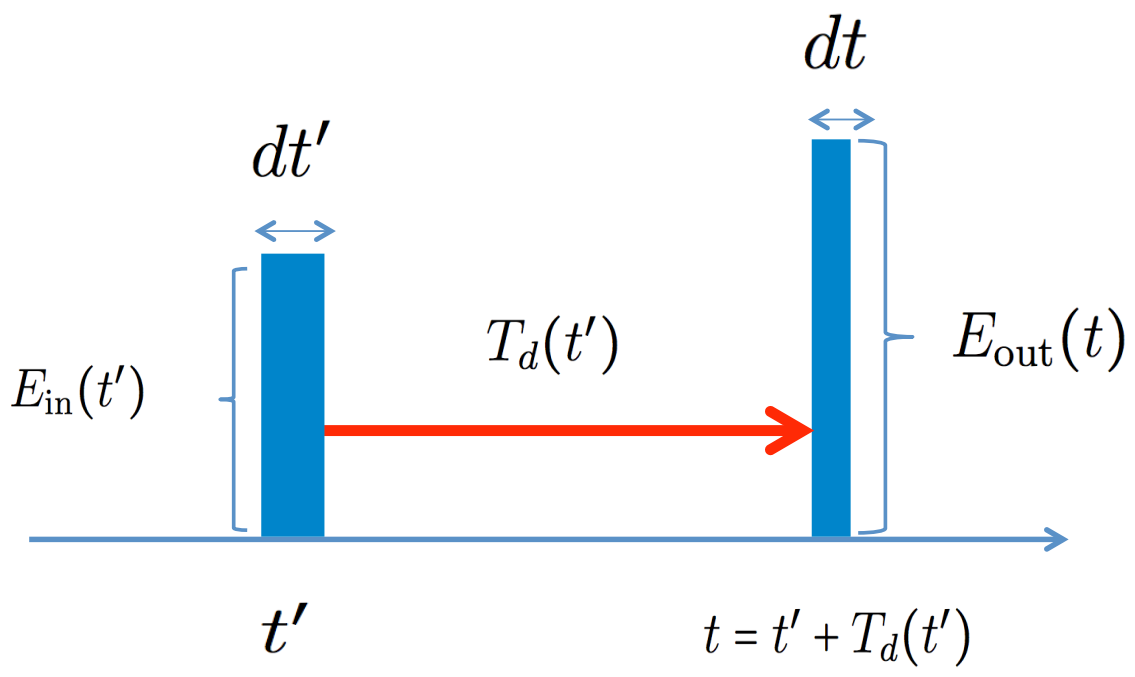

Figure 3.3: Electric slice mapping during a time transformation process. A input electric slice of height of $E_{\text {in }}\left(t^{\prime}\right)$, width of $d t^{\prime}$ is transferred to a corresponding output electric field slice of height of $E_{\text {out }}(t)$, width of $d t$.

It is also important to keep in mind that the slice duration itself may change because of the temporal mapping. Figure. 3.3 illustrates the electric slice mapping in a time transformation process. Two simple rules apply here. First of all, the temporal locations for the input and output slice is related by the time transformation relation [Eq. (3.15)]. Secondly, the "slice area" $E(t) d t$ should be conserved during this linear mapping process:

$$
E_{\text {out }}(t) d t=E_{\text {in }}\left(t^{\prime}\right) d t^{\prime}
$$

Therefore, once the refractive index profile of a medium $n(t)$ is known, the time transformation can be found using Eqs. (3.15) and (3.16), and the output electric field can be obtained by further using Eq. (3.17). 
Note that several assumptions are made in the above analysis. First of all, the spatial effects are ignored for simplicity, assuming that the pulse is propagating inside an optical waveguide (e.g., a fiber). Secondly, reflections at the boundaries as well as within the medium are neglected, assuming that dynamic or nonlinear index changes are relatively small. The issue of reflection is discussed in the next chapter, and it is found that reflections can be appropriately incorporated into the time transformation method. Last but not the least, the medium is assumed to respond instantaneously, i.e., the medium is assumed to be non-dispersive. With the presence of dispersion, each temporal slice would experience broadening, and the mapping model would break down accordingly. It seems that time transformation cannot be applied to dispersive media. However, it would be shown in the next subsection that dispersion can be incorporated into the time transformation method by appropriately combining time transformation method with linear system theory.

To better explain our time transformation method, we show in Fig. 3.4 the time transformation in Eq. (3.15) for three types of non-dispersive dielectric media that are referred to as a static linear, a dynamic linear, and a nonlinear Kerr medium. In the special case of a static linear medium with a constant refractive index $n_{0}$, all temporal slices travel at a same constant speed of $v_{0}=c / n_{0}$. Therefore, a slice that enters the medium at $t^{\prime}$ would exit the slab at time

$$
t=t^{\prime}+T_{d 0}
$$

where $T_{d 0}=n_{0} L / c$ is the transit time for this slab that is a constant and independent 
on the input slice time $t^{\prime}$. In this situation, the input-output mapping in Eq. (3.18) is linear and corresponds to a straight line with a slope of $45^{\circ}$ [blue-dotted curve in Fig. 3.4]. Clearly, such a static linear medium only shifts each pulse slice by a constant time delay $T_{d 0}$. As a consequence, the electric field $E_{\text {in }}(t)$ of an input pulse is transformed at the output end to become

$$
E_{\text {out }}(t)=E_{\text {in }}\left(t-T_{d 0}\right) .
$$

Except for a constant phase shift, the transmitted electric field is exactly the same as the input electric field, which meets the expectation for a static linear medium.

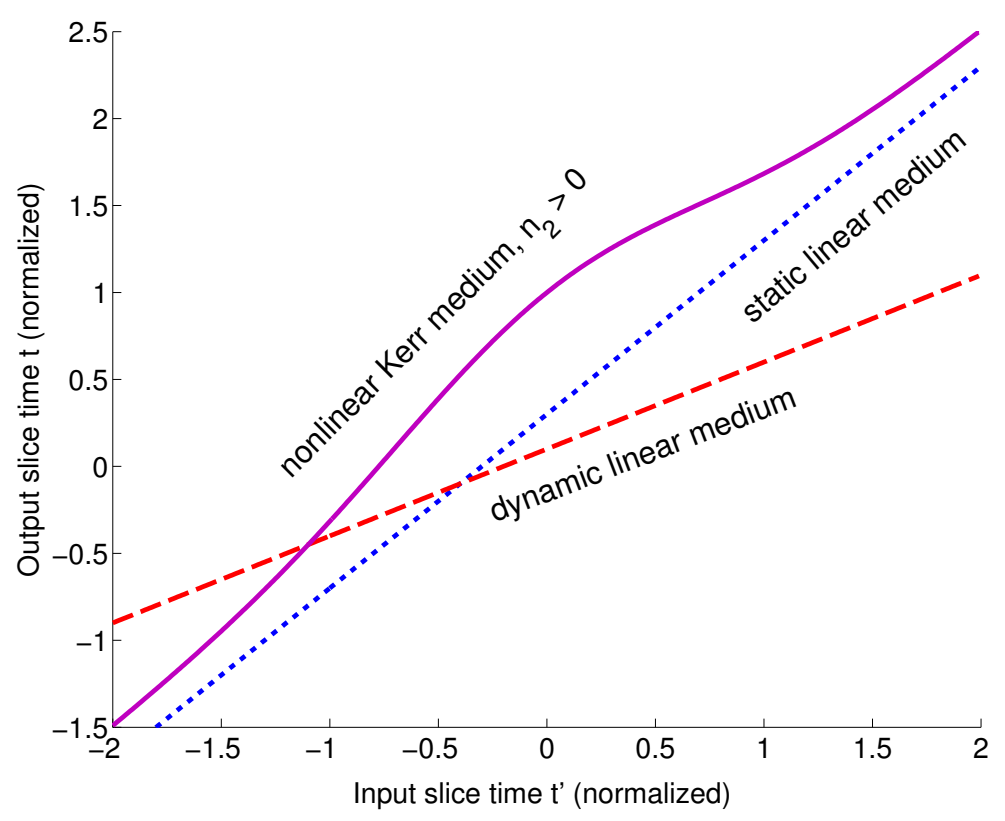

Figure 3.4: Temporal mapping in the cases of a static linear medium (bluedotted), a dynamic linear medium (red-dashed), and a nonlinear Kerr medium (purple-solid). Depending on how refractive index changes with time, the temporal mapping can be linear or nonlinear. 
In general cases, the refractive index of a medium can change with time. For example, the refractive index for a Kerr nonlinear medium changes locally following the optical pulse inside [57]. In the context of adiabatic wavelength conversion, the refractive index of the whole linear medium is assumed to change uniformly in time [1]. A direct consequence of such a dynamic refractive index is that different temporal slices of the electric field now travel at different speeds as they pass through such a dynamic medium. Accordingly the transit delay time $T_{d}$ is no longer constant and depends on when light enters the medium and how the refractive index evolves with time. As would be shown in this thesis, the time transformation method could be applied to these two types of media (referred as dynamic linear media and nonlinear media) as well. Depending on whether $T_{d}$ is a linear or nonlinear function of $t^{\prime}$, this time transformation can be linear or nonlinear. For example, the time transformation for a dynamic linear medium and a Kerr nonlinear medium are plotted with the reddashed and purple-solid curves, respectively in Fig. (3.4). The majority of this thesis would be devoted to showing how to apply the time transformation method to these media, and to study the optical pulse propagation behavior inside such dynamic media.

\subsection{Inclusion of chromatic dispersion}

As is discussed in Section 2.3, most of the dielectric media do not have an instantaneous respond and exhibit certain amount of dispersion. In reality, dispersion cannot 
be ignored and should be incorporated into time transformation method so that it can be applied to real experimental conditions.

In order to include the impact of dispersion, let us go back to the linear system theory first. A dispersive linear medium, whose refractive index changes with frequency, can be thought of as a time-invariant linear system. The impulse response function of such a medium can be found easily in the frequency domain. If we consider a specific frequency component of the electric field with frequency $\omega$, it acquires a phase shift of $\phi=\beta(\omega) L$ as it propagates through such a linear medium of length $L$. Therefore, the transmitted spectrum $\tilde{E}_{\text {out }}(\omega)$ can be related to the input $\tilde{E}_{\text {in }}(\omega)$ through

$$
\tilde{E}_{\text {out }}(\omega)=\exp [i \beta(\omega) L] \tilde{E}_{\text {in }}(\omega)
$$

where $\beta(\omega)=n(\omega) \omega / c$ is the propagation constant of light inside a medium with the refractive index $n(\omega)$ and $c$ is the speed of light in vacuum. It follows from Eq. (3.2) that the frequency response function for a dispersive medium is $H(\omega)=\exp [i \beta(\omega) L]$. The impulse response function can be obtained by taking the inverse Fourier transform of its frequency response function

$$
h\left(t-t^{\prime}\right)=\frac{1}{2 \pi} \int_{-\infty}^{\infty} \exp \left[i \beta(\omega) L-i \omega\left(t-t^{\prime}\right)\right] \mathrm{d} \omega
$$

A simple check of Eq. (3.21) has been performed in Section 3.2, where we have shown that the impulse response function for a non-dispersive static linear medium is

$$
h\left(t-t^{\prime}\right)=\delta\left(t-t^{\prime}-T_{d 0}\right)
$$


The extension of the time transformation method to the dispersive cases can be done by noticing that the variable inside the impulse response function [Eq. (3.22)] is a time transformation. Therefore, the impact of a dynamic refractive index $n(t)$ can be incorporated by appropriately modifying the time transformation term inside the impulse response function $h\left(t, t^{\prime}\right)$. For this purpose, a new time variable $t_{1}$ is introduced as a function of the input time variable $t^{\prime}$ :

$$
t_{1}=F\left(t^{\prime}\right)=t^{\prime}+T_{d}\left(t^{\prime}\right)
$$

Clearly, $h\left(t, t^{\prime}\right)$ in Eq. (3.4) can now be written as $h\left(t-t_{1}\right)$ and Eq. (3.4) itself becomes

$$
E_{\text {out }}(t)=\int_{-\infty}^{\infty} h\left(t-t_{1}\right) E^{\prime}\left(t_{1}\right) J\left(t_{1}\right) \mathrm{d} t_{1}
$$

where $E^{\prime}\left(t_{1}\right)=E_{\text {in }}\left(t^{\prime}\right)$ and the Jacobian of this time transformation is given by

$$
J\left(t_{1}\right)=\mathrm{d} t^{\prime} / \mathrm{d} t_{1}=\left(1+\mathrm{d} T_{d} / \mathrm{d} t^{\prime}\right)^{-1}
$$

with $T_{d}\left(t^{\prime}\right)$ is the transit time for the electric field slice at input time $t^{\prime}$ and can be calculated using Eq. (3.16). Notice that this Jacobian comes from the transformation of coordinate axis from $t^{\prime}$ to $t_{1}$, and is related to our previous discussion that the areas of the electric field slices are conserved [Eq. (3.17)].

Comparing Eq. (3.24) with Eq. (3.4), a major modification is the time transformation given in Eq. (3.23). More specifically, the temporal delay of the electric field slice is reflected in the relation $E^{\prime}\left(t_{1}\right)=E_{\text {in }}\left(t^{\prime}\right)$, while the modification to the slice width is done through the Jacobian. Thanks to time transformation, Eq. (3.24) becomes 
very general, and can be applied to any dynamic medium $[75,76]$, even to a medium that is nonlinear [77-79].

Before we apply this method to different media, it is necessary to discuss the numerical implementation of this method. Notice that Eq. (3.24) is a convolution after the time transformation, it can be calculated efficiently in the Fourier domain using the convolution theorem. More specifically, the time transformation Eq. (3.23) and the corresponding Jacobian Eq. (3.25) are firstly calculated in the temporal domain. Then the impact of dispersion can be taken care of easily in the frequency domain. In Appendix B, a MATLAB code is attached as an example that shows how to apply time transformation method to nonlinear dispersive media. 


\section{Adiabatic Wavelength}

\section{Conversion in Dynamic Linear}

\section{Media}

This chapter applies the time transformation method to dynamic linear media to study the phenomenon of adiabatic wavelength conversion (AWC) that was observed inside dynamic linear photonic structures, such as a waveguide and a resonator. Specifically, a universal formula for the optical field at the output end of a timevarying medium is presented. This formula agrees with the known features of AWC, such as a shift in central frequency and a change in the electromagnetic energy. More importantly, this formula reveals additional changes to the pulse such as temporal broadening or compression, as well as spectral broadening. The impact of the details of how refractive index changes is studied and it is shown that AWC does not depend on the details of $n(t)$ as long as the initial and final refractive indices are fixed. The issue of reflection and transmission is also discussed, and the corresponding coefficients 
are found for a temporal boundary.

\subsection{Analytical results for dynamic linear media}

Let us now apply the time transformation method to a dynamic linear medium. To begin, we assume the specific temporal profile for the refractive index shown in Fig. 4.1. More specifically, the refractive index begins to change from its initial value of $n_{1}$ at time $T_{i}$, follows some arbitrary function $n(t)$ until the final time $T_{f}$ such that $n\left(T_{f}\right)=n_{2}$, and stays at $n_{2}$ after that. It is also assumed that the pulse is launched into such a dynamic medium before $T_{i}\left(t_{i}<T_{i}\right)$ and leaves after $T_{f}\left(t_{o}>T_{f}\right)$ so that the pulse experiences the whole changing process. Under these conditions, the integral in Eq. (3.16) takes the form:

$$
\int_{t^{\prime}}^{T_{i}} \frac{c}{n_{1}} \mathrm{~d} \tau+\int_{T_{i}}^{T_{f}} \frac{c}{n(\tau)} \mathrm{d} \tau+\int_{T_{f}}^{t^{\prime}+T_{d}\left(t^{\prime}\right)} \frac{c}{n_{2}} \mathrm{~d} \tau=L .
$$

The time-dependent transit time $T_{d}\left(t^{\prime}\right)$ in this general case can be calculated from Eq. (4.1) and has the following form:

$$
T_{d}\left(t^{\prime}\right)=(1-s) t^{\prime} / s+T_{d 0}
$$

where an important stretching factor $s=n_{1} / n_{2}$ is introduced [note that this stretching factor is essentially the constant Jacobian discussed in Eq. (3.25)] and $T_{d 0}$ is an effective transit time delay that is expressed fully as

$$
T_{d 0}=n_{2} L / c+T_{f}-T_{i} / s-n_{2} \int_{T_{i}}^{T_{f}}[1 / n(\tau)] \mathrm{d} \tau
$$




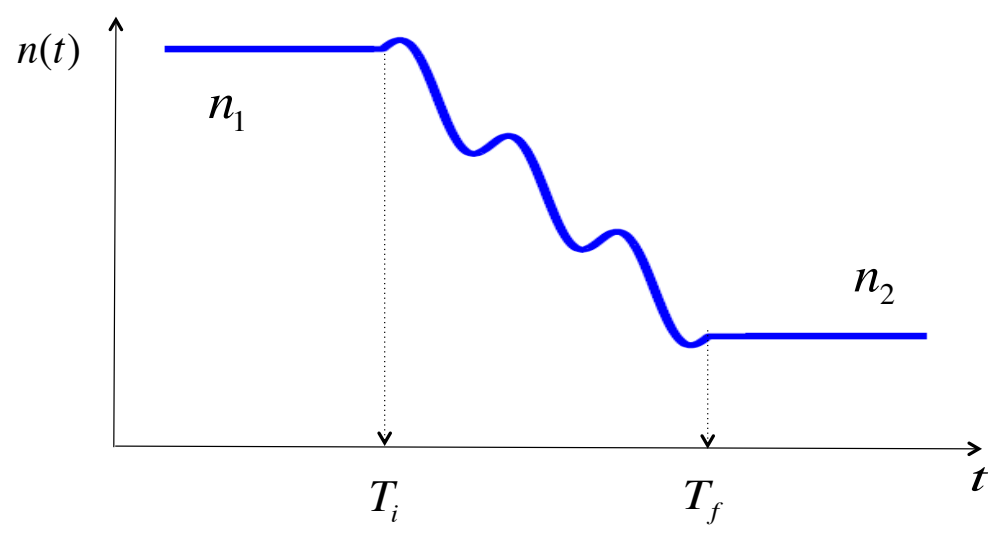

Figure 4.1: Temporal profile for the refractive index in an AWC process: it changes from a stable value of $n_{1}$ beginning at $T_{i}$, following some arbitrary function, until it reaches to a new stable value $n_{2}$ after $T_{f}$.

Equation (4.3) shows that the effective transit time $T_{d 0}$ has the same value for all input electric field slices (independent of $t^{\prime}$ ). If the refractive index does not change during the time interval $T_{f}-T_{i}$, then $n_{2}=n_{1}, s=1$, and $T_{d}\left(t^{\prime}\right)$ is reduced to the constant value $n_{1} L / c$, as expected for the time-invariant case.

One can accordingly write out the time-transformation relation for such a dynamic medium:

$$
t \equiv t^{\prime}+T_{d}\left(t^{\prime}\right)=t^{\prime} / s+T_{d 0}
$$

Since both $s$ and $T_{d 0}$ are constant, the time transformation given by Eq. (4.4) is linear, and we plot it using solid-red curve in Fig. 4.2 for $s=0.8$, and $T_{d 0}=0.7$. As shown in this figure, the time transformation for a dynamic linear medium is a straight line. The special case of a static linear medium is also plotted here using dotted-blue curve for comparison. The change for a dynamic medium is that the slope for this line is 


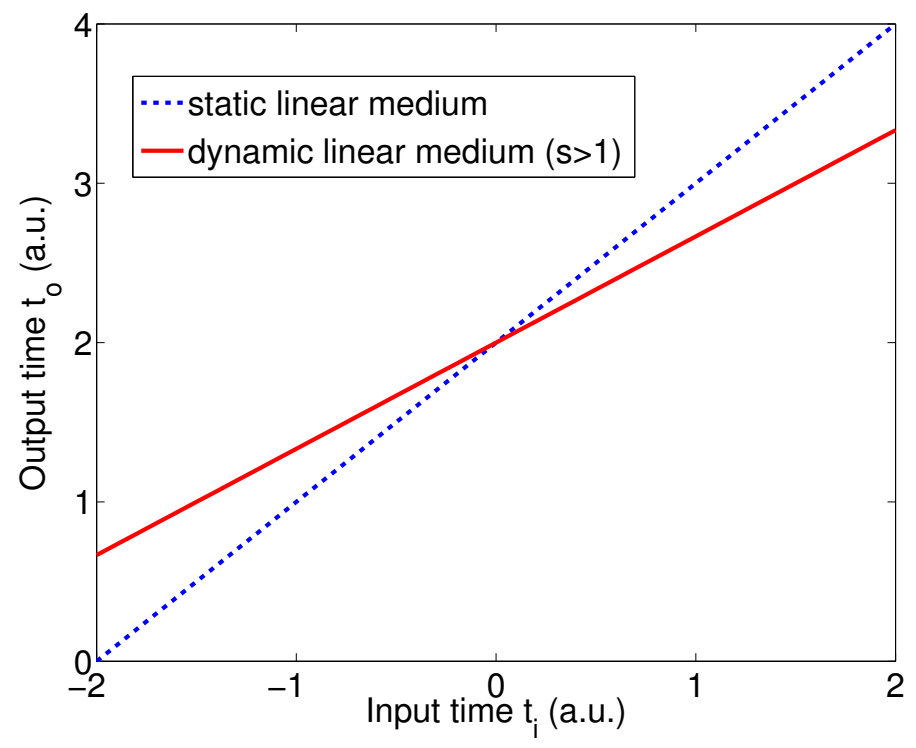

Figure 4.2: Time transformation for a dynamic linear medium (solid-red) whose index decreases $\left(n_{1}>n_{2}\right)$. The case for a static linear medium (dotted-blue) is also plotted for comparison.

not 1 . For the case of a medium with decreasing refractive index shown in Fig. 4.1, $n_{1}>n_{2}$, and the slope is $1 / s=n_{2} / n_{1}<1$. Such a deviation of the slope from 1 leads to time compression (in the case of $s<1$ ) or time stretching (in the case of $s>1$ ).

In order to see the impact of such a linear time-transformation on an optical pulse, one can substitute Eq. (4.4) into the the time transformation method in Eq. (3.17), and obtain an analytical expression in the time domain [Eq. (4.5)]. The corresponding frequency domain formula [Eq. (4.6)] is obtained by its Fourier transform:

$$
\begin{gathered}
E_{\text {out }}(t)=s E_{\text {in }}\left(s t-s T_{\mathrm{d} 0}\right), \\
\tilde{E}_{\text {out }}(\omega)=\tilde{E}_{\text {in }}(\omega / s) \exp \left(-i \omega T_{\mathrm{d} 0}\right) .
\end{gathered}
$$


These formulas show that the major effect of changing the medium's refractive index in a dynamic fashion manifests on the optical pulse through a stretching parameter $s=n_{1} / n_{2}$ (that depends only on the ratio of the initial and final values of the refractive index) and on an effective transit-time delay $T_{\mathrm{d} 0}$. More specifically, the " $s$ " factor in front of $E_{\text {in }}$ in Eq. (4.5) modifies the amplitude of the electric field. While the " $s$ " factor associated with time $t$ inside $E_{\text {in }}$ has two major effects, noticing that the electric field can be written as a combination of a slowly varying envelope and a fast optical oscillation part. "s" changes the duration of the pulse through the envelop $A(t)$, and also changes the duration of every single optical cycle through the carrier frequency term $e^{-i \omega t}$.

\subsection{Adiabatic wavelength conversion}

As an example of how the stretching factor $s$ affects optical pulses, we consider an optical Gaussian pulse:

$$
\begin{gathered}
E_{\text {in }}(t)=E_{0} e^{-t^{2} /\left(2 T_{0}^{2}\right)-i \omega_{1} t}, \\
\tilde{E}_{\text {in }}(\omega)=E_{0} T_{0} \sqrt{2 \pi} e^{-T_{0}^{2}\left(\omega-\omega_{1}\right)^{2} / 2},
\end{gathered}
$$

where $T_{0}$ is a measure of the pulse width and $\omega_{1}$ is the carrier frequency. The corresponding output field can be obtained directly by applying Eqs. (4.5) and (4.6) to obtain

$$
E_{\text {out }}(t)=s E_{0} e^{-s^{2}\left(t-T_{\mathrm{d} 0}\right)^{2} /\left(2 T_{0}^{2}\right)-i s \omega_{1}\left(t-T_{\mathrm{d} 0}\right)},
$$




$$
\tilde{E}_{\text {out }}(\omega)=E_{0} T_{0} \sqrt{2 \pi} e^{-T_{0}^{2}\left(\omega-s \omega_{1}\right)^{2} /\left(2 s^{2}\right)-i \omega_{1} T_{\mathrm{d} 0}}
$$

For the special case $s=1$, where the medium is time-invariant, neither the temporal shape $P(t)=|E(t)|^{2}$ nor the spectrum $P(\omega)=|\tilde{E}(\omega)|^{2}$ of the pulse changes, a case shown in Fig. 4.3 by solid-green curves, where we used $E=\sqrt{P} e^{i \phi}$. The only effect of the time-invariant medium is to delay the pulse by $T_{d o}$ and to induce a phase offset and linear slope in the temporal and spectral domains respectively, as depicted by the solid-green curves in Fig. 4.3.

In the general time-variant case, we assume a linear variation of $n(\tau)$ for $s \neq 1$, while the interval $T_{f}-T_{i}=0.5 n_{1} L / c$ is preserved, and plot the result for both $s>1$ and $s<1$ in Fig. 4.3. As shown in Fig. 4.3, the output pulse remains Gaussian in shape but its carrier frequency changes to $\omega_{2}=s \omega_{1}$. Depending on whether the refractive index is increased $\left(n_{2}>n_{1}\right.$ and $\left.s<1\right)$ or decreased $\left(n_{2}<n_{1}\right.$ and $\left.s>1\right)$, the frequency of the light can be red-shifted or blue-shifted. In addition, this frequency shift is linearly proportional to the change in refractive index. This frequency shift matches previous FDTD and experimental studies of AWC [1,2]. The magnitude of central frequency shift depends on the ratio $s=n_{1} / n_{2}$ and is relatively small in practice, typically $<0.01 \%$, but recently a shift of $0.2 \%$ has been demonstrated in Ref. [3]. For an optical frequency $\nu \sim 200 \mathrm{THz}$, a $0.2 \%$ change in frequency is about $0.4 \mathrm{THz}$, which can be easily measured experimentally.

Besides this frequency shift, time transformation method also reveals new features of AWC process. This spectral shift is accompanied with temporal reshaping of the 


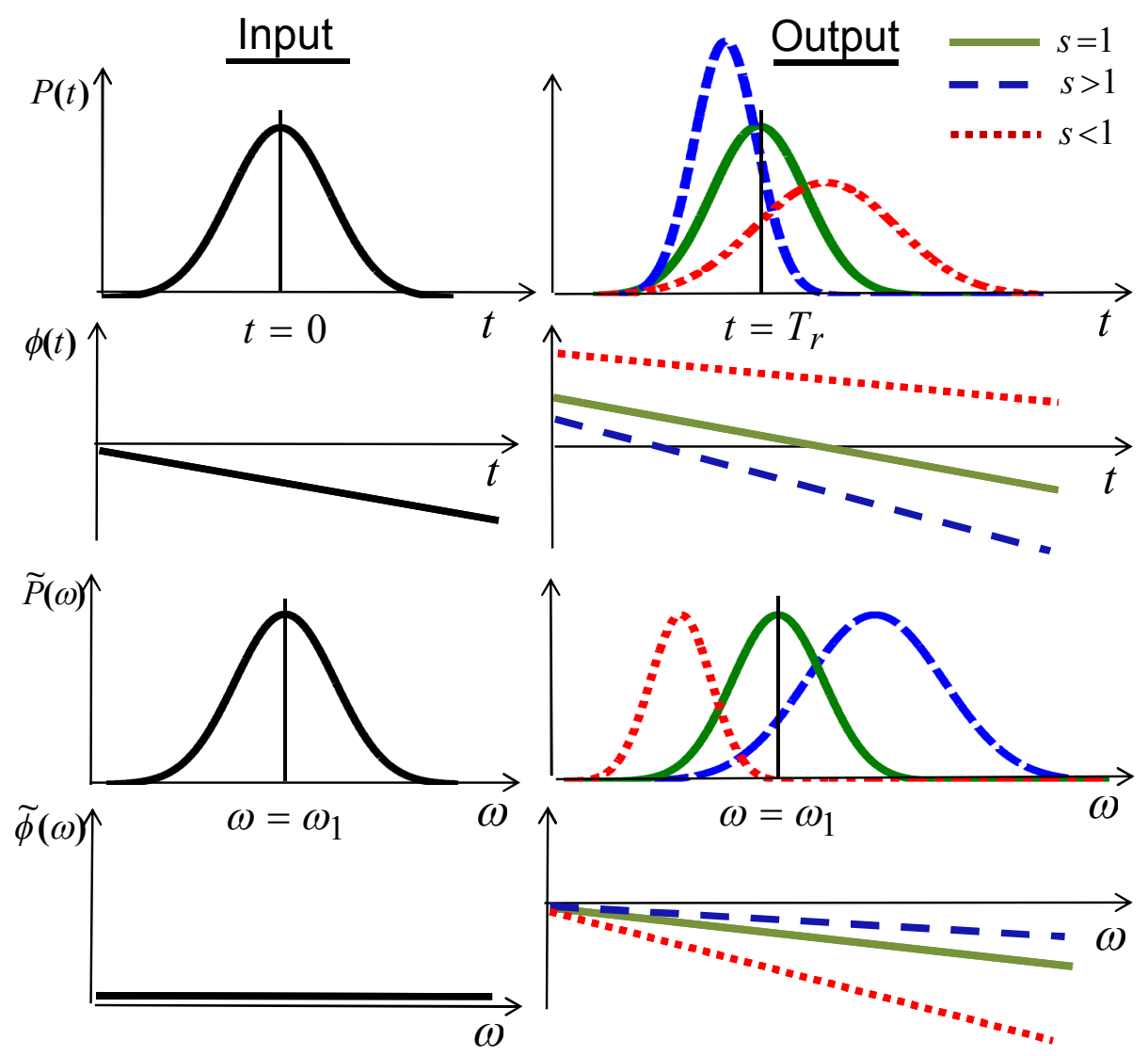

Figure 4.3: Input and output temporal powers, temporal phases, and spectral powers, spectral phases of a input Gaussian pulse for a AWC process. The dashed (blue), solid (green), and dotted (red) curves correspond to stretching factors of $s=3 / 2,1$, and $2 / 3$, respectively.

pulse. It is evident from Eqs. (4.7) and (4.9) that the temporal pulse width changes from $T_{0}$ to $T_{0} / s$. Moreover, the spectral width $\left(W_{0}=1 / T_{0}\right)$ changes to $W_{0}^{\prime}=s W_{0}$, as evident from Eqs. (4.8) and (4.10). A blueshift of the central wavelength $(s>1)$ results in temporal pulse compression, amplitude enhancement, and spectral broadening, as depicted by the dashed-blue curves in Fig. 4.3. On the other hand, a redshift $(s<1)$ 
results in temporal pulse broadening, amplitude reduction, and spectral compression, as depicted by the dotted-red curves in Fig. 4.3. Although a $0.2 \%$ changes (or less) in the temporal and spectral width of a pulse may not be easy to observe, the temporal power scales as $s^{2}$, making the asymmetry between red and blue AWC shifts easily measurable. Furthermore, the nonlinear effects scale as $s^{4}$ or higher order and should be much more noticeable.

The conserved physical quantity for AWC is the so-called action, defined as $J=$ $U / \omega[5]$, where $U$ is the pulse energy. Physically, $J$ is proportional to the number of photons contained in the pulse, a conserved quantity expected for AWC. This invariance in action (not energy) was first brought to light in the FDTD simulation in Ref. [1] and is the reason why this wavelength-conversion phenomenon is called adiabatic wavelength conversion. Using our universal formulas [Eqs. (4.5) and (4.6)], this conservation law can be straightforwardly shown:

$$
J_{\text {out }}=\frac{\int_{-\infty}^{\infty}\left|s E_{\text {in }}\left(s t-s T_{d 0}\right)\right|^{2} d t}{s \omega}=\frac{\int_{-\infty}^{\infty}\left|E_{\text {in }}(t)\right|^{2} d t}{\omega}=J_{\text {in }} .
$$

One important feature of the time-transformation method is that it works directly with the electric field and and dose not assume an envelop function. Therefore, evolution of the electric field can be deduced without any approximation. In fact, the evolution of the electric field itself can provide much more information than the evolution of the temporal and spectral powers of the pulse. To illustrate the role of temporal mapping for a dynamic linear medium, Fig. 4.4 shows how the electric field associated with a four-cycle optical pulse (with a Gaussian envelop, pulse width 
about $5 \mathrm{fs}$ ) changes after it passes through the medium, using an unrealistically large value of $\mathrm{s}=1.5$ for the scaling factor.
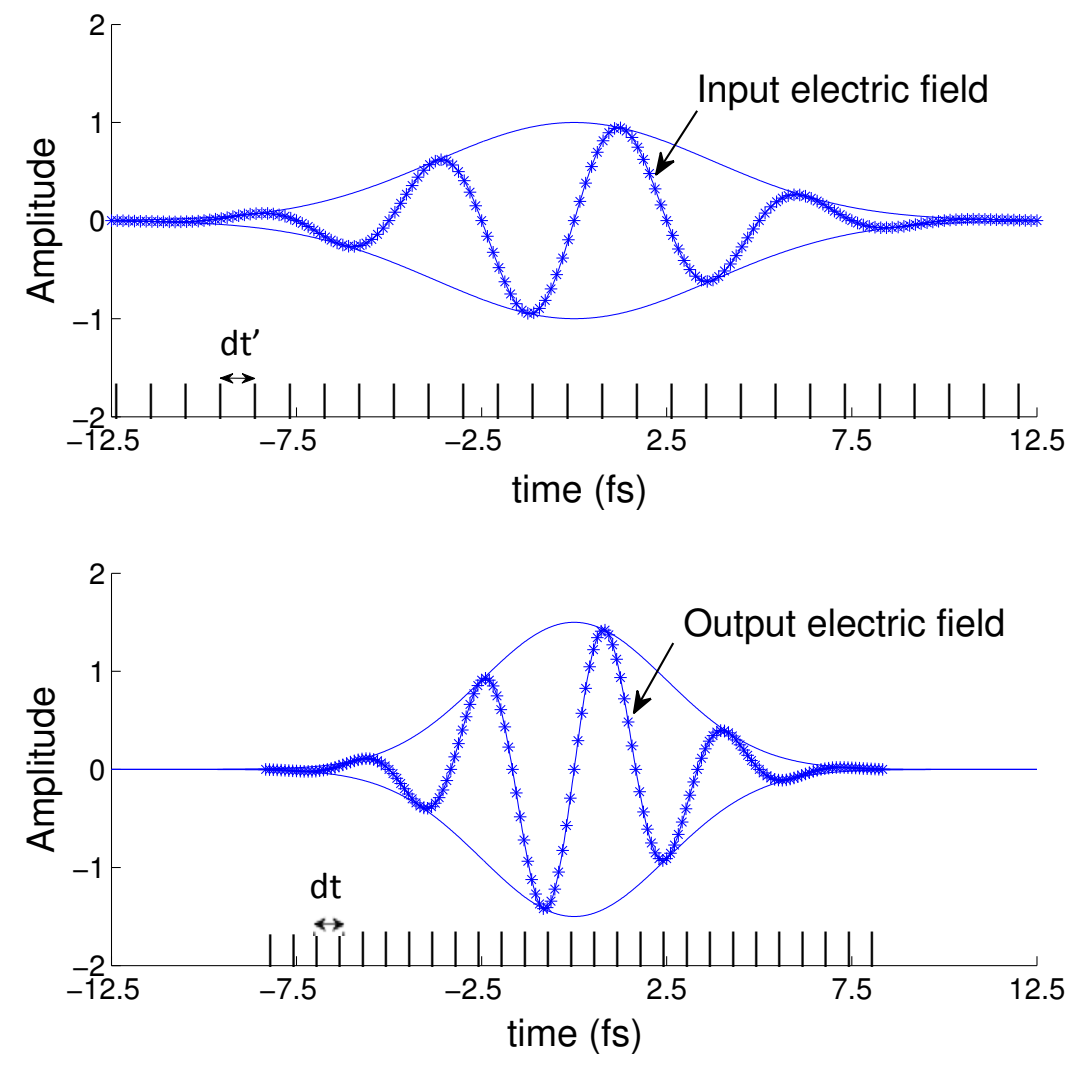

Figure 4.4: Input and output electric fields (crosses) and their corresponding envelops (solid curve) associated with a four-cycle optical Gaussian pulse after propagating through a dynamic medium with $s=1.5$.

As shown in the top part of Fig. 4.4, the input pulse is divided into temporal slices of duration $d t^{\prime}$. At the output end, this duration shrinks by the factor of 1.5 , resulting in the output electric field shown in the bottom part of Fig. 4.4. As seen there, the linear temporal mapping, $d t^{\prime}=s d t$, deforms the output pulse in such a way that the amplitude of the electric field is uniformly increased by a factor of $s$ because 
of a temporal compression of the pulse. Temporal compression of the electric field also leads to an increase of the carrier frequency of the optical pulse by the factor of $s$. The linear time transformation in Eq. (4.4) can also be thought as a dynamic medium to function like a time lens [80]. In the case of $s>1$, the time lens focuses the electric field associated with an optical pulse in the temporal domain, while the pulse would be broadened by the medium for the case of $s<1$. Although the pulse is compressed or broadened, the number of optical cycles contained in this pulse does not change during this process. In the spectral domain, this compression or broadening of the electric field manifests as a increase or decrease in the carrier frequency of the pulse. Now, the picture behind the adiabatic wavelength conversion is clear: it is just a consequence of the linear temporal mapping by a dynamic linear medium.

AWC does cause a nonlinear change in the temporal phase for chirped input pulses, a previously unnoticed effect. Specifically, a quadratic phase factor $e^{-i c t^{2}}$, where $c$ is a constant linear chirp changes to at the output $e^{-i s^{2} c\left(t-T_{\mathrm{d} 0}\right)^{2}}$ from Eq. (4.5). AWC causes the output chirp to become $s^{2} c$. In the blue-shifted case $(s>1)$, the chirp is enhanced, whereas in the red-shifted case $(s<1)$, the chirp is reduced. These changes in the temporal phase lead to change in pulse width upon further propagation down fiber-optic transmission system. 


\subsection{Impact of time-dependent $n(t)$}

As shown in Ref. [1], changes in the pulse wavelength $(\Delta \lambda)$ and energy $(\Delta U)$ do not depend on details of how refractive index is modified for an AWC process. Figure 4.5 considers the impact of different functions $n(t)$ on pulse propagation. Specifically, four different refractive index profiles are considered: an instantaneous change (solidblue); two linear changes with fast (dashed-red) and slow (dotted-black) changing rates; an arbitrary change (dotted-dash purple). All these four cases have the same value of initial $\left(n_{1}\right)$ and final $\left(n_{2}\right)$ refractive index. From Eq. (4.1), it follows that the temporal slice delay in all four cases of $n(t)$ shown in Fig. 4.5 is given by

$$
T_{\mathrm{dk}}\left(t^{\prime}\right)=(1-s) t^{\prime} / s+T_{\mathrm{d} 0 \mathrm{k}}
$$

where $s=n_{1} / n_{2}$ and the effective transit time $T_{\mathrm{d} 0 \mathrm{k}}$ is obtained from Eq. (4.3) using the appropriate form of $n(t)$ for the $k_{\mathrm{th}}$ scenario $(k=1$ to 4$)$. Since the same stretching factor $s$ appears, the temporal and spectral power shapes of the output pulse are identical in all four cases, as depicted in Fig. 4.5. This conclusion that the frequency shift only depends on the initial and final refractive index and is independent of the details of how the refractive index changes agrees with the numerical results obtained by the Maxwell-equation-based FDTD method [1].

However, the effective transit time does depends on details of the temporal profile of the refractive index, as is clearly seen from Eq. (4.3). Therefore, the output pulse does experience a temporal delay that depends on $n(t)$, even for the same value of 


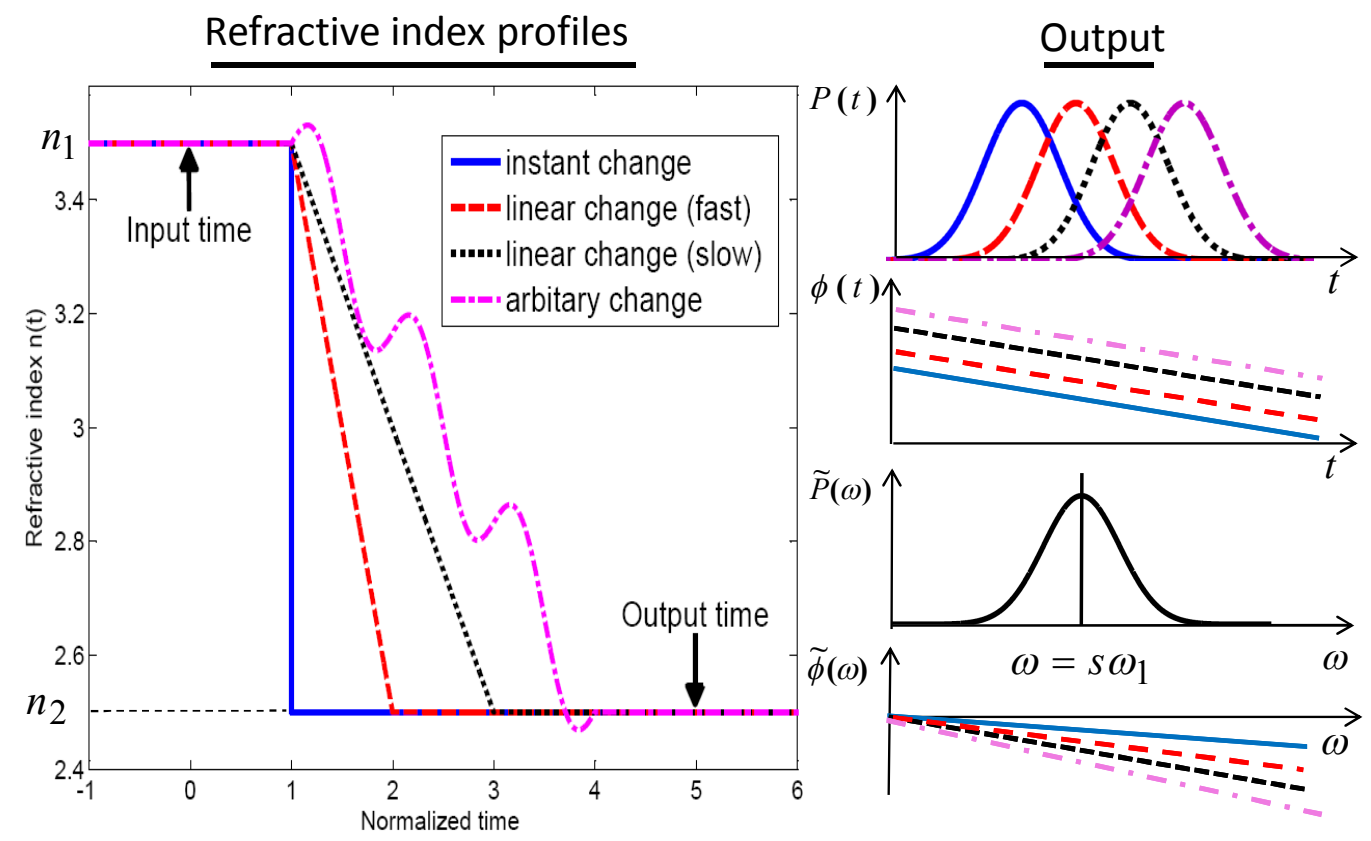

Figure 4.5: Four dynamic refractive index profiles and the corresponding output pulses. In all cases, the refractive index is initially $n_{1}$ and becomes $n_{2}$ after the change. The output pulses are identical in temporal shape and spectral power, but have different time delays.

$s$, as depicted in Fig. 4.5. This dependence on the dynamic index profile may be pursued as a means of controlled optical buffering [81]. Also, pulse delay depends on the "direction" of frequency shift; for the blue-shifted case $(s>1)$ in Fig. 4.5, the pulse is advanced, while for the red-shifted case $(s<1)$, the pulse would be delayed. This can be easily understood since increasing the refractive index would decrease the light speed, and it would take longer time for the pulse to propagate through the medium. The optical phases shown in Fig. 4.5 also depend on $n(\tau)$. The central frequency $s \omega$ (the slope of the linear temporal phase) are the same for all four cases. 
The slope of the spectral phase $\tilde{\phi}(\omega)$ changes with $n(\tau)$, corresponding to the change in the time delay of the temporal pulse.

\subsection{Reflection and transmission of electromagnetic waves at a temporal boundary}

As discussed in Chapter 2, the investigation of AWC phenomena began as early as 1958 [7]. In addition to the shift of the light frequency, it was also predicted that a backward-propagating wave would be generated. The generation of a reflected wave is expected noticing that the impedance changes as well for a dynamic medium whose refractive index changes with time. However, there seems to be no definite answer to the question "what are the reflection and transmission coefficients for electromagnetic waves at a temporal boundary?"

Published literature contains two different sets of expressions for the reflection and transmission coefficients of EM waves at a temporal boundary. One set was derived by Morgenthaler in his 1958 paper using specific boundary conditions [7] and has been used in many other studies since then $[11,12,18,44,46]$. The other set was presented in 1975 using different boundary conditions in the context of plasma [10] and has also been used by others $[14,15,26,29,82]$. So far, there has been little discussion of the connection between these two different sets of reflection and transmission coefficients.

This section focuses on the reflection and transmission of light at a temporal 
boundary. It is shown that two different physical processes contribute to them. One process is related to impedance mismatch, while the other results from temporal scaling due to a sudden change in the speed of light at the temporal boundary. The temporal scaling is related to the parameter $s$ of this chapter. Time transformation method reveals the origin of difference in the two sets of reflection and transmission coefficients found in the literature and shows under what conditions they can be used in practice.

Figure 4.6 shows what happens to a plane wave, polarized along the $x$ axis and propagating along the $z$ axis, as it approaches a temporal boundary located at $t=t_{0}$, where the refractive index changes from $n_{1}$ to $n_{2}$. Before the boundary $\left(t<t_{0}\right)$, the plane wave is propagating at a speed $c / n_{1}$. At the boundary $\left(t=t_{0}\right)$, its speed changes to $c / n_{2}$, and at the same time a reflected wave is created with its electric and magnetic field oriented as shown in Fig. 4.6. The reflected wave will be generated only if the medium impedance changes at the temporal boundary, i.e., if $\eta_{1}=\left(\mu_{1} / \epsilon_{1}\right)^{1 / 2}$ is different from $\eta_{2}=\left(\mu_{2} / \epsilon_{2}\right)^{1 / 2}$, where $\epsilon$ and $\mu$ represent the dielectric permittivity and magnetic permeability of the medium, respectively, and the subscripts 1 and 2 denote these quantities before and after the temporal boundary located at $t=t_{0}$.

In the following discussion, the electric and magnetic fields before $t=t_{0}$ are denoted as $\mathbf{E}_{\text {in }}$ and $\mathbf{H}_{\text {in }}$. After the index change, the transmitted and reflected electric fields are denoted by $\mathbf{E}_{t}$ and $\mathbf{E}_{r}$, respectively, with a similar notation for the magnetic field. Transmission and reflection at a temporal boundary can be characterized by 

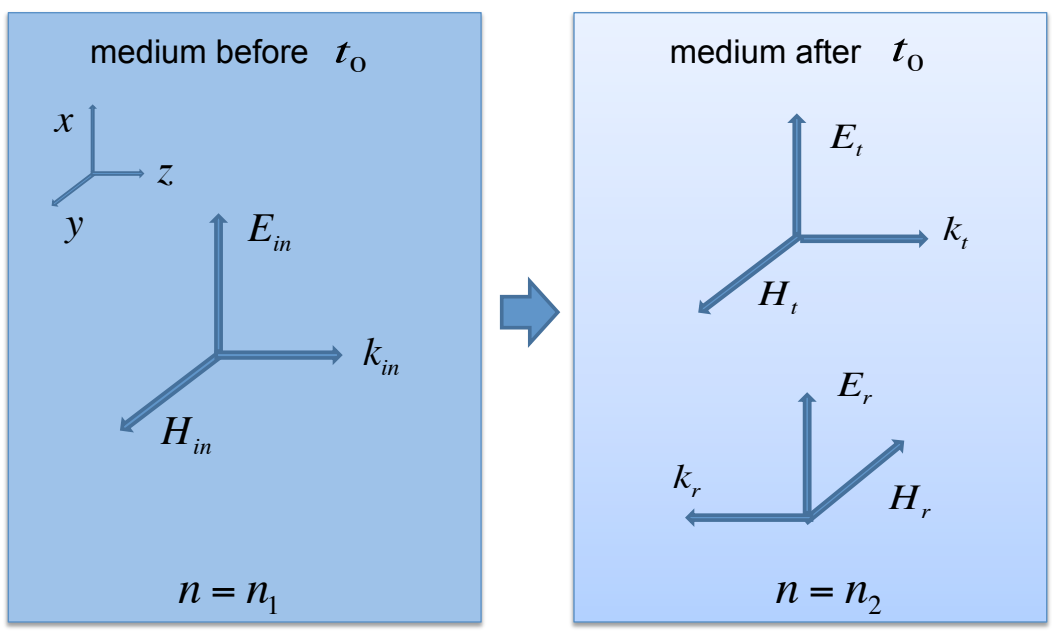

Figure 4.6: Schematic illustration of reflection and transmission of an electromagnetic fields at a temporal boundary where the refractive index changes from $n_{1}$ (left panel) to its final value $n_{2}$ (right panel) at $t=t_{0}$.

coefficients similar to Fresnel's equations at a spatial boundary. However, there is considerable confusion in the published literature, as two different sets of expressions exist for the transmission and reflection coefficients.

Both cases start from Maxwell's equations. The two sets of expressions differ in their choice of the boundary conditions that the four fields $\mathbf{D}, \mathbf{B}, \mathbf{E}$, and $\mathbf{H}$ must satisfy across the temporal boundary. One approach argues that the quantities $\mathbf{D}$ and B should remain continuous across the temporal boundary in view of the presence of their time derivatives in Eqs. (2.1c) and (2.1d):

$$
\mathbf{D}\left(t=t_{0}^{-}\right)=\mathbf{D}\left(t=t_{0}^{+}\right), \quad \mathbf{B}\left(t=t_{0}^{-}\right)=\mathbf{B}\left(t=t_{0}^{+}\right) .
$$

These boundary conditions ensure that Maxwell's equations remain valid for all times, including the instant $t_{0}$ when the refractive index changes suddenly. In this case, the 
transmission and reflection coefficients for the electric field are found to be

$$
\begin{aligned}
& r_{\mathrm{DB}} \equiv \frac{E_{r}}{E_{\mathrm{in}}}=\frac{1}{2}\left(\frac{\epsilon_{1}}{\epsilon_{2}}-\frac{\sqrt{\mu_{1} \epsilon_{1}}}{\sqrt{\mu_{2} \epsilon_{2}}}\right), \\
& t_{\mathrm{DB}} \equiv \frac{E_{t}}{E_{\mathrm{in}}}=\frac{1}{2}\left(\frac{\epsilon_{1}}{\epsilon_{2}}+\frac{\sqrt{\mu_{1} \epsilon_{1}}}{\sqrt{\mu_{2} \epsilon_{2}}}\right) .
\end{aligned}
$$

These equations were first derived in Ref. [7] and have been been used extensively since then $[11,12,18,44,46]$.

In the second approach, one insists on the continuity of the electric and magnetic fields, $\mathbf{E}$ and $\mathbf{H}$, and assumes that they cannot change abruptly at $t_{0}$ when the refractive index of the medium changes at a temporal boundary:

$$
\mathbf{E}\left(t=t_{0}^{-}\right)=\mathbf{E}\left(t=t_{0}^{+}\right), \quad \mathbf{H}\left(t=t_{0}^{-}\right)=\mathbf{H}\left(t=t_{0}^{+}\right)
$$

This assumption leads to another set of expressions for the reflection and transmission coefficients for the electric field:

$$
\begin{aligned}
& r_{\mathrm{EH}} \equiv \frac{E_{r}}{E_{\mathrm{in}}}=\frac{1}{2}\left(1-\frac{\sqrt{\mu_{2} \epsilon_{1}}}{\sqrt{\mu_{1} \epsilon_{2}}}\right), \\
& t_{\mathrm{EH}} \equiv \frac{E_{t}}{E_{\mathrm{in}}}=\frac{1}{2}\left(1+\frac{\sqrt{\mu_{2} \epsilon_{1}}}{\sqrt{\mu_{1} \epsilon_{2}}}\right) .
\end{aligned}
$$

Clearly, only one set of these coefficients can be right as Eqs. (4.13) and (4.16) cannot be satisfied simultaneously at a temporal boundary. The question is which one? Equation (4.13) is the correct choice, as also expected intuitively on the physical ground. To fully understand what happens at a temporal boundary, one needs to consider the special case in which the refractive index is changed such that the 
impedance $\eta$ does not change across the temporal boundary $\left(\eta^{2}=\mu_{1} / \epsilon_{1}=\mu_{2} / \epsilon_{2}\right)$, and no light is reflected $\left(r_{\mathrm{DB}}=0\right)$. Naïvely one would think that $t_{\mathrm{DB}}$ must be 1 to meet the "expected" $100 \%$ of transmission through the temporal boundary. [Indeed $t_{\mathrm{EH}}=1$, and this might be the reason that Eqs. (4.17) and (4.18) were adopted.] However, Eq. (4.15) predicts $t_{\mathrm{DB}} \neq 1$. To claim that Eq. (4.13) is the correct choice, one needs to understand the physical origin for changes in the amplitude of the transmitted electric field in the case of matched impedances.

A clue is found by noting that the two sets of transmission and reflection coefficients are not independent, rather they can be related as

$$
r_{\mathrm{DB}}=-\frac{n_{1}}{n_{2}} r_{\mathrm{EH}}, \quad t_{\mathrm{DB}}=\frac{n_{1}}{n_{2}} t_{\mathrm{EH}},
$$

where $n_{i}=\sqrt{\epsilon_{i} \mu_{i} /\left(\epsilon_{0} \mu_{0}\right)}$ with $i=1$ or 2 . The same ratio $n_{1} / n_{2}$ was also found to occur in Eq. (4.5), where $s=n_{1} / n_{2}$ is the time-dilation factor related to change in the speed of light after the temporal boundary. The physical origin of the timedilation factor $s$ is related to time transformation [77] that maps the input field to the output field. More precisely, each temporal slice of the electric field is either "stretched" or "compressed" depending on whether the speed of light decreases or increases after passing the temporal boundary. Equation (4.5) shows that such time dilation is accompanied with a linear scaling of the field amplitude by the factor $s$. More importantly, the amplitude modification factor $s$ is the factor relating two sets of transmission and reflection coefficients in Eq. (4.19). Thus, we conclude that the transmission coefficient in Eq. (4.15) is the product of two factors that have different 
physical origins. Indeed, it can be written as $t_{\mathrm{DB}}=s t_{\mathrm{EH}}$. The factor $s$ is due to the time dilation, whereas the factor $t_{\mathrm{EH}}$ has its origin in the impedance mismatch across a temporal boundary. The use of "wrong" boundary conditions in Eq. (4.16) misses completely the factor $s$. Clearly, the boundary conditions in Eq. (4.13) must be used since they capture both physical effects. The minus sign appearing in Eq. (4.19) can be understood from our time transformation method by noting each slice of the reflected electric field reverses its propagation direction upon reflection; this time reversal results in the factor -1 through time transformation.

Previous works did point out changes in the optical frequency across a temporal boundary, which is one of the consequences of time dilation, but most of them focused on the continuous-wave (CW) case. Therefore, "stretching" or "compression" of the electric field was not discussed. Although numerical simulations in Ref. [44] showed changes in the width of an optical pulse in dynamic media, the physical reason behind it was not discussed in that work. Here the time transformation method shows that time dilation should not be ignored, as it modifies the amplitudes for the transmitted electric by a factor of $s$. This factor is also needed to ensure the validity of the boundary conditions given in Eq. (4.13).

Since Maxwell's equations contain the whole physics, any predictions from the time transformation method should agree with the solution of these equations. To verify this, Maxwell's equations are solved numerically using the FDTD method discussed in Section 2.1.4. For simplicity, a one-dimensional propagation problem is considered 
where the electric field is in the form of a 4-cycle pulse (with its peak amplitude normalized to 1 ) and propagates in the $z$ direction as a plane wave (without any diffraction in the transverse plane). A temporal boundary is assumed to exist when the medium parameters $\epsilon$ and $\mu$ change instantaneously.

In case 1 , the permittivity of the whole medium is increased uniformly from $\epsilon_{1}=2 \epsilon_{0}$ to $\epsilon_{2}=1.5625 \epsilon_{1}$ but the permeability is kept unchanged $\left(\mu_{1}=\mu_{2}=\mu_{0}\right)$. Such a change increases the refractive index by $25 \%(s=0.8)$. Since the impedance of the medium also changes, a reflected wave must be generated in this case. The electric field obtained numerically after the temporal boundary is plotted in Fig. 4.7. The peak amplitude of the transmitted field is 0.72 . One can also clearly see that a part of the pulse energy is reflected back. The peak amplitude of the reflected field is 0.08 , and its phase is shifted by $\pi$. Using the parameter values employed in numerical simulations, it is not hard to find that Eqs. (4.14) and (4.15) give the correct amplitude for both the reflected and transmitted fields.

In case 2 , both the permittivity and permeability of the medium are changed in such a way that the impedance of the medium does not change at the temporal boundary even though the refractive index does change. More specifically, the parameters are chosen as $\epsilon_{2}=1.25 \epsilon_{1}$ and $\mu_{2}=1.25 \mu_{0}=1.25 \mu_{1}$ so that the refractive index of the medium changes again by $25 \%$. Figure 4.8 shows the electric field obtained numerically in this situation. As one might have expected, FDTD simulations confirm that no reflections occur in the presence of impedance matching. The amplitude of the 


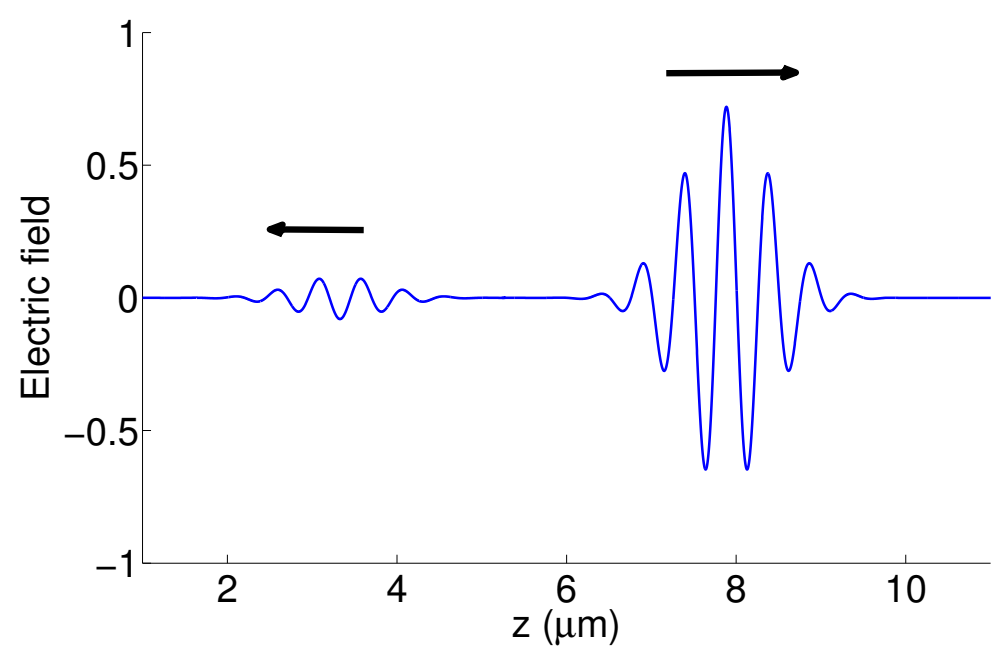

Figure 4.7: Electric field of a 4-cycle pulse after propagation across a temporal boundary. Reflected wave is generated because of impedance mismatch. The amplitudes of the reflected and transmitted fields agree with Eqs. (4.14) and (4.15).

transmitted field is still reduced in this case, with a peak value of 0.8 , which agrees with the prediction of Eq. (4.15). Clearly, this reduction is not related to energy transfer to a reflected wave because no reflection occurs. As is discussed earlier, this reduction is related to the prediction of time transformation method in Eq. (4.5). Indeed, one can immediately verify that $s=n_{1} / n_{2}=0.8$, in agreement with the numerically obtained value of 0.8 in Fig. 4.8. Amplitude changes of transmitted light due to stretching or compressing of temporal slices was first pointed out Ref. [75].

To take a closer look at how the transmitted field changes in the preceding two cases, Fig. 4.9 plots the transmitted electric fields in case 1 (blue curve) and case 2 (yellow curve) on top of the input electric field (green curve). The prediction of 


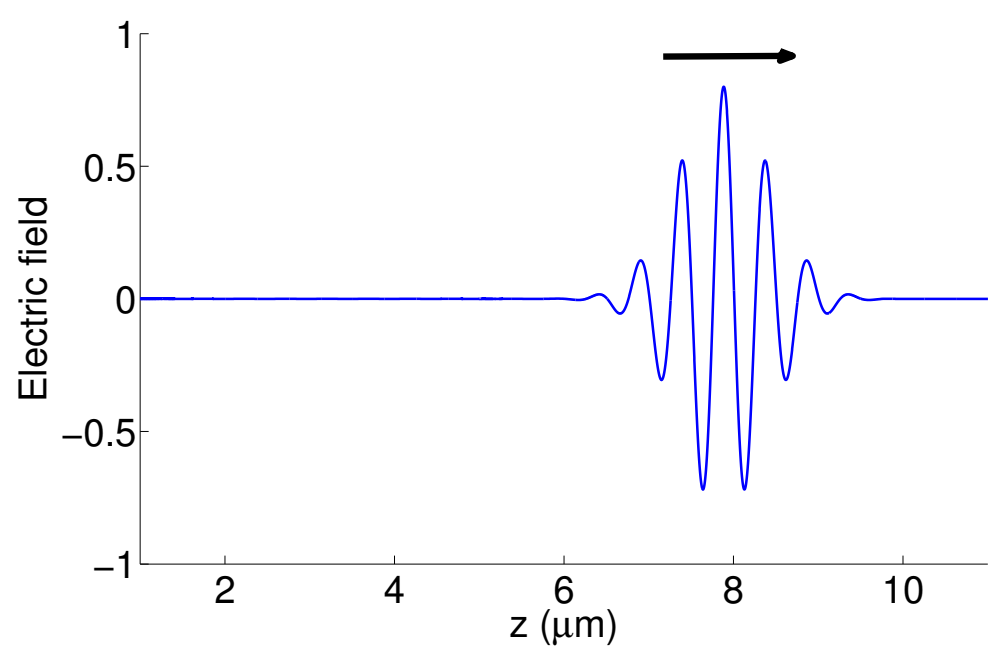

Figure 4.8: Electric field of a 4-cycle pulse after propagation across a temporal boundary with impedance matching. No electric field is reflected back. The amplitudes of the reflected and transmitted fields agree with Eqs. (4.14) and (4.15).

time transformation method [Eq. (4.5)] in case 2, shown with a dotted black curve, is in excellent agreement with FDTD simulations. More specifically, the amplitude of the field is modified by a factor of 0.8 ; the carrier frequency is also modified by the same factor with the corresponding change in the duration of an optical cycle. When the impedance is not matched across the temporal boundary (case 1), the transmitted field looks almost the same as in case 2. The only difference is that its amplitude is further reduced from 0.8 to 0.72 . This reduction is solely due to reflection and its magnitude is given by $t_{\mathrm{EH}}=0.9$ given in Eq. (4.18). The FDTD simulations shown in Figs. 4.7-4.9 confirm the conclusion reached earlier based on the time-transformation method that Eqs. (4.13)-(4.15) provide correct description 


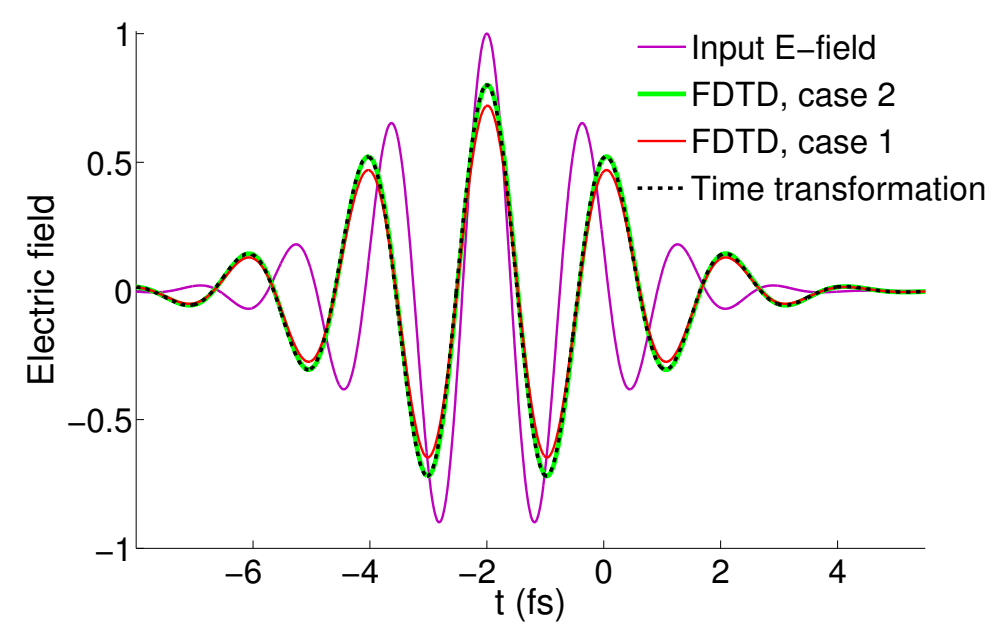

Figure 4.9: Comparison of transmitted electric fields in cases 1 (blue curve, impedance changes) and 2 (yellow curve, impedance matched). Dotted black curve shows the analytic prediction based on Eq. (4.5). The input electric field is shown for comparison with a green curve. FDTD simulations confirm and agree fully with the predictions of the time transformation method.

of reflection and transmission of an EM field at a temporal boundary.

We should clarify that the analysis here treats reflection and transmission of electromagnetic waves at a temporal boundary of a nondispersive medium. It should be stressed out that this model does not apply to plasma whose permittivity is inherently dispersive. In this case, the reflection and transmission coefficients derived in Refs. $[10,14,15,26,29,82]$ are the correct ones. 


\section{Adiabatic Wavelength}

\section{Conversion in Dynamic Linear}

\section{Resonators}

In this chapter, the analysis in Chapter 4 for dynamic linear media is extended to study adiabatic wavelength conversion (AWC) inside dynamic linear resonators. The time transformation method shows that the adiabatic wavelength shift results from a scaling of time by a factor that depends on both the magnitude and the direction of index change. This method is first applied to the case in which pulses short compared with the round-trip time of the resonator are transmitted and the refractive index is changed instantaneously. Then, the case of input pulses longer than the roundtrip time is considered, and it is shown that an adiabatic wavelength shift is always accompanied with significant changes in both the shape and the spectrum of output pulses. How such temporal and spectral changes depend on externally controllable parameters such as the magnitude and the speed of index change and detuning of the 
input pulse from the cavity resonance is discussed in detail. These result should find applications in the area of optical signal processing with resonant photonic structures based on microrings or photonic crystals.

\subsection{Impulse response for dynamic resonators}

In recent years, new kinds of optical resonators, based on silicon microrings and photonic crystals, are being used to make optical devices with numerous potential applications. An example is provided by the phenomenon of adiabatic wavelength conversion (AWC) occurring inside an optical resonator whose refractive index is forced to change with time while an optical pulse is transmitted through it $[1,2,38]$. In contrast to conventional static resonators, it is common to think of such a resonator as being dynamic. This chapter focuses on such dynamic resonators. Although they can have different configurations, their properties can be studied by considering a generic FabryPerot (FP) resonator [83]. Historically, dynamic FP resonators with moving mirrors were first considered during the 1960s in the context of mode locking [84]. Such resonators have continued to attract attention and have found applications in the fields of spectroscopy and interferometry [85-89]. Recently, dynamic resonators with dynamic refractive index became a hot topic in the context of AWC. As shown in Chapter 4, a novel time transformation method can successfully explain AWC happening inside a traveling-waveguide. One might ask, can this method be applied to resonators as well? The answer is yes, and the extension is fairly easy. 


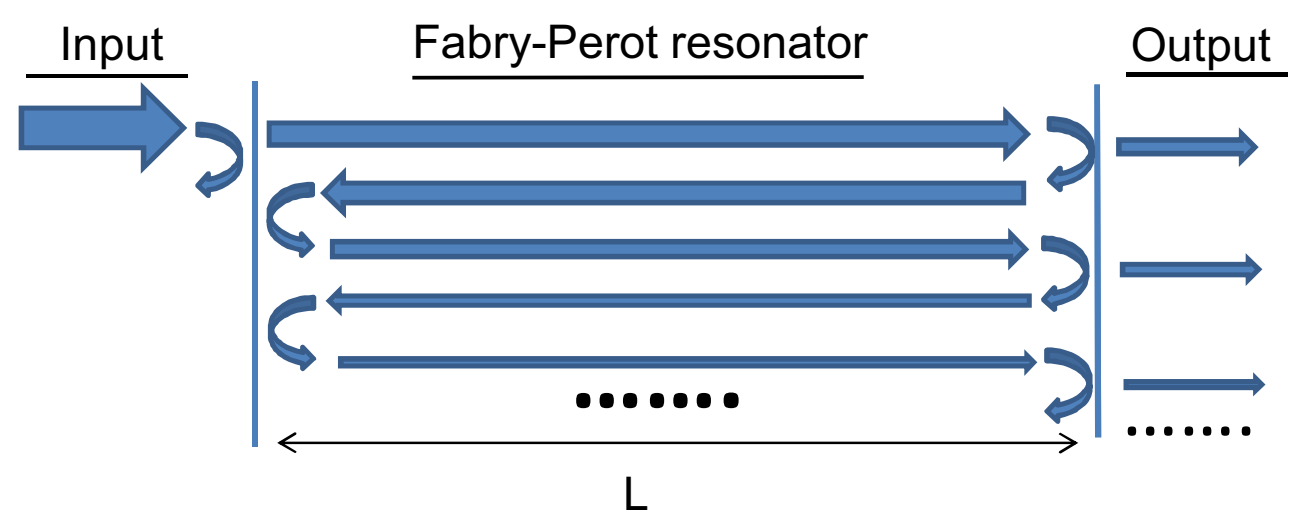

Figure 5.1: Schematic illustration of multiple round trips within a Fabry-Perot resonator. For a single input electric field slice, the output is a sequence of electric field slices with decreasing amplitude, due to the multi-bouncing of light by the two end mirrors.

A dielectric slab can be converted into a FP resonator by assuming that its two facets located at $z=0$ and $z=L$ act as mirrors of reflectivity $R$. Since light is now forced to make multiple passes within the resonator, for a input of a single pulse, the output field is not in the form of a single pulse, as is the case for a traveling waveguide. Rather, as seen in Fig. 5.1, it is composed of a sequence of output fields of decreasing amplitudes resulting from successive passes within the resonator.

In the picture of time transformation, the previous mapping of one input electric field slice to one output slice for traveling waveguides now becomes the mapping of one input slice to multiple output slices. The temporal locations of these slices are determined by the round trip time of the cavity $T_{r}$, which is no longer a constant for a general dynamic cavity. The amplitudes of these output slices are affected by two 
factors. The first one comes from the time dilation discussed in Chapter 4, which is due to the change in the speed of light. The second one is related to the mirror reflection, which is constant $R$ for each roundtrip.

Following linear system theory of Section 2.1, it is a simple matter to show that the impulse response function of a FP resonator is given by

$$
h\left(t, t^{\prime}\right)=(1-R) \sum_{m=0}^{\infty} R^{m} \delta\left[t-t^{\prime}-T_{\mathrm{dm}}\left(t^{\prime}\right)\right]
$$

where $m=0,1,2 \ldots$ for successive round trips within the resonator. The quantity $T_{\mathrm{dm}}\left(t^{\prime}\right)$ is the transit time for the pulse slice that enters the resonator at time $t^{\prime}$ and leaves after $m$ round trips. It is readily obtained from Eq. (3.16) by replacing $L$ with $(2 m+1) L$

$$
\int_{t^{\prime}}^{t^{\prime}+T_{\mathrm{dm}}\left(t^{\prime}\right)}[c / n(\tau)] \mathrm{d} \tau=(2 m+1) L
$$

where $L$ is the physical length of the resonator. Once the impulse response function is known, a dynamic FP resonator for an arbitrary functional form of $n(t)$ and an arbitrary input field can be fully characterized by substituting Eq. (5.1) into Eq. (3.4):

$$
E_{\text {out }}(t)=(1-R) \sum_{m=0}^{\infty} R^{m} \int_{-\infty}^{\infty} \delta\left[t-t^{\prime}-T_{\mathrm{dm}}\left(t^{\prime}\right)\right] E_{\text {in }}\left(t^{\prime}\right) \mathrm{d} t^{\prime}
$$

Before considering the dynamic situation, let us apply Eq. (5.3) to a static FP resonator with a constant refractive index $n_{0}$. In such a cavity, light travels at a constant speed of $c / n_{0}$, and the transit time simply becomes: $T_{\mathrm{dm}}=(1+2 m) T_{r 0} / 2$, where $T_{r 0}=2 n_{0} L / c$ is the round-trip time. It follows from Eq. (5.1) that the impulse 
response function of a static FP resonator is given by

$$
h(t)=(1-R) \sum_{m=0}^{\infty} R^{m} \delta\left[t-(1+2 m) T_{r 0} / 2\right] .
$$

Notice that the impulse response function $h\left(t, t^{\prime}\right)$ now becomes a single-variable function $h\left(t-t^{\prime}\right)$ for a static resonator (a time-invariant system). Taking the Fourier transform of Eq. (5.4) and summing up all the resulting series, the frequency-domain transfer function becomes

$$
H(\omega)=(1-R) e^{i \omega T_{r 0} / 2} /\left(1-R e^{i \omega T_{r 0}}\right) .
$$

This is identical to the well-known transfer function of static FP resonators [83]. The agreement between the time-domain and frequency-domain approaches is expected on physical grounds.

\subsection{Propagation of short optical pulses}

This section firstly focus on the case where input pulses are short compared to the round-trip time of the resonator. To begin with, the refractive index is assumed to change instantaneously from its initial value of $n_{1}$ to $n_{2}$ at time $T_{1}$ and to stay at $n_{2}$ after that, i.e.,

$$
n(t)= \begin{cases}n_{1} & \left(t<T_{1}\right), \\ n_{2} & \left(t \geq T_{1}\right) .\end{cases}
$$


If optical pulse enters the resonator after $T_{1}$, it does not experience the refractive index change and the resonator is effectively static. What is interested is the case where pulses enter the resonator before the refractive index changes.

The time $T_{d m}\left(t^{\prime}\right)$ appearing in Eq. (5.1) can be calculated analytically for this index-change model by writing the integral in Eq. (5.2) in the form

$$
\int_{t^{\prime}}^{T_{1}} \frac{c}{n_{1}} \mathrm{~d} \tau+\int_{T_{1}}^{t^{\prime}+T_{\mathrm{dm}}\left(t^{\prime}\right)} \frac{c}{n_{2}} \mathrm{~d} \tau=(2 m+1) L
$$

Both integrals can be performed easily, and the result is

$$
T_{\mathrm{dm}}\left(t^{\prime}\right)=(1-s) t^{\prime} / s+T_{\mathrm{em}}
$$

where $s=n_{1} / n_{2}$ is the scaling factor and $T_{\mathrm{em}}$ is defined as

$$
T_{\mathrm{em}}=(2 m+1) n_{2} L / c+(1-1 / s) T_{1} .
$$

If there is no refractive index change, then $n_{2}=n_{1}, s=1$, and $T_{\mathrm{em}}$ is reduced to the value of $(2 m+1) n_{1} L / c$ expected on physical grounds. Compared to the time-invariant case, the most important feature of Eq. (5.8) is a rescaling of the slice time $t^{\prime}$ to $t^{\prime} / s$. As will be seen later, this scaling affects the optical pulse in several different ways.

Once $T_{\mathrm{dm}}\left(t^{\prime}\right)$ is known, the transfer function $h\left(t, t^{\prime}\right)$ is known from Eq. (5.1). By substituting it into the linear system theory, the output field can be obtained in the following form

$$
E_{\text {out }}(t)=(1-R) \sum_{m=0}^{\infty} R^{m}\left[s E_{\text {in }}\left(s t-s T_{\mathrm{em}}\right)\right] .
$$

Equation (5.10) shows that the effect of changing the medium's refractive index in a dynamic fashion manifests through a simple scaling parameter $s=n_{1} / n_{2}$ that only 
depends on the ratio of the initial and final values of the refractive index. As will be seen later, this scaling leads to pulse compression or broadening depending on whether $s>1$ or $s<1$. Notice that the amplitude is also altered by the same factor $s$.

As an illustration, this general analysis is first applied to the specific case of a Gaussian input pulse with the carrier frequency $\omega_{1}$. The input field is then given by

$$
E_{\text {in }}(t)=E_{0} \exp \left[-t^{2} /\left(2 T_{0}^{2}\right)-i \omega_{1} t\right]
$$

where the pulse width $T_{0}<n_{1} L / c$ so that the entire pulse can fit within the resonator. To make this calculation relevant for silicon micro-rings, a relatively short FP resonator with $L=10 \mu \mathrm{m}, n_{1}=3.5$ and $R=0.8$ is considered. Since the round-trip time is only $233 \mathrm{fs}, T_{0}$ is chosen to be a small value of $60 \mathrm{fs}$. The carrier frequency of the pulse is chosen to be $192.86 \mathrm{THz}$ so that it coincides with one of the cavity modes and corresponds to a wavelength of $1555.56 \mathrm{~nm}$.

Figure 5.2 shows shapes and spectra of the output pulse in two cases in which the refractive index of the resonator medium increases (blue solid curves) or decreases (red dashed curves) by $5 \%$ from its initial value of 3.5 at $T_{1}=0.25$ ps. The input pulse and spectrum is also shown for comparison (green dot-dashed curves). Multiple pulses seen in Fig. 5.2(a) result from the sum in Eq. (5.10); index changes not only shift the exit time of subpulses but also lead to changes in their widths. As seen in Fig. 5.2(b) and (c), the output spectrum is affected the most by index changes. The ultrashort input pulse has a broad enough spectrum that it excites 5 cavity modes in both the static (dotted green curve) and dynamic (solid blue and dashed red curves) 
cases. However, spectral peaks exhibit a blue or red shift of about $10 \mathrm{THz}$ in the two dynamic cases. The shifted peaks correspond to new cavity mode frequencies $\nu_{k}=k c /\left(2 n_{2} L\right)$, where $k$ is an integer, after the refractive index is changed from $n_{1}$ to $n_{2}$. Since no nonlinear effects are included in our analysis, the linear nature of the AWC process is self-evident.
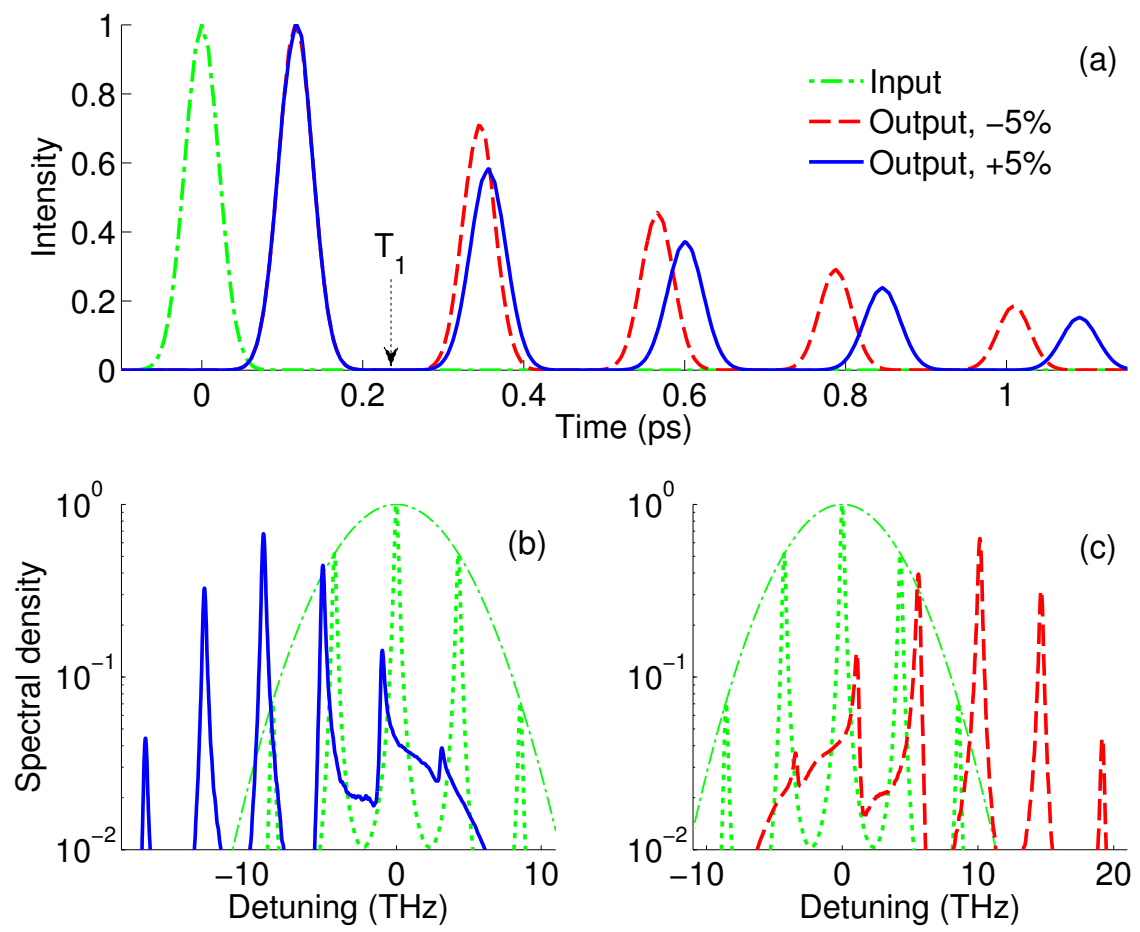

Figure 5.2: Short-pulse (60 fs) propagation for an instantaneous change in refractive index of $-5 \%$ (blue) and $+5 \%$ (red) at time $T_{1}=250$ fs. The round-trip time is 233 fs. Detuning $\Delta \nu$ is defined as $\nu-\nu_{0}$, where $\nu_{0}$ is the input frequency. (a) The index change alters the width, amplitude, and delay of temporal pulses after $T_{c}$. The index change shifts the comb-like input spectrum (green curve) to higher $[+5 \%,(\mathrm{~b})]$ and lower $[-5 \%,(\mathrm{c})]$ frequencies at the FP output. 
Although spectral shifts resulting from AWC in dynamic resonators are well known $[1,2,38]$, it has not been realized that spectral shape also changes considerably. As seen in Fig. 5.2(b) and (c), the output spectrum exhibits considerable asymmetry. This asymmetry is due to the asymmetric nature of the index-change process with respect to the pulse center. More precisely, the refractive index is changed after the pulse has already entered the resonator and a part of its energy has already leaked out of the resonator. This feature implies that different parts of the temporal output should have different spectral contents (i.e., the pulse is chirped). A spectrogram is often used to display time-dependent spectral changes [59]. It is constructed by using a sampling function $W(t, \tau)$, before measuring the spectrum, that selects different pulse slices. It is calculated using

$$
S(\omega, \tau)=\left|\int_{-\infty}^{\infty} W(t, \tau) E_{\text {out }}(t) e^{i \omega t} \mathrm{~d} t\right|^{2}
$$

Figure 5.3 shows the spectrogram calculated using a Gaussian-shape sampling function in the case of $-5 \%$ reduction in the refractive index. One can clearly see that the first output field ( $m=0$ term) is centered at the original frequency because index has not yet been changes, while the remaining output fields $(m>0)$ are all centered at the frequency shifted by about $10 \mathrm{THz}$ because they exit the resonator after the index has been changed.

In constructing Figs. 5.2 and 5.3, the refractive index was changed by 5\%, a relatively large amount that is hard to realize experimentally, where the index changes are often produced by injecting free carriers into the mode volume within a silicon 


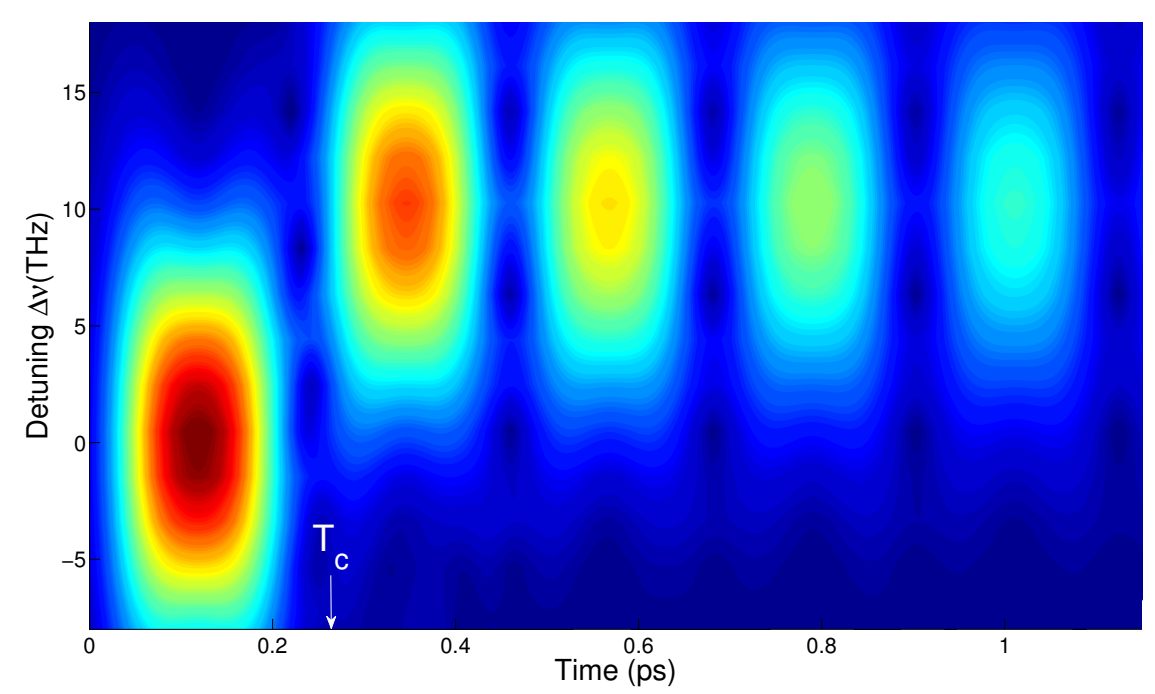

Figure 5.3: Spectrogram of the output field in the case of $-5 \%$ index change at $T_{c}=250 \mathrm{fs}$. A shift in central frequency of about $10 \mathrm{THz}$ is clearly seen after the first subpulse.

waveguide [2]. In practice, index changes are typically below $0.5 \%$. Calculations were also performed for such small amount of refractive index change and the results show that changes in the time domain are relatively minor and barely noticeable. In the spectral domain, although the spectral shifts are much lower $(<1 \mathrm{THz})$, the output spectrum remains asymmetric and exhibits features that should be measurable experimentally.

One interesting point to note is that the response of a dynamic FP resonator does not depend on the carrier frequency for short pulses. This is readily seen from the time-domain approach. The transmitted pulse is a sequence of subpulses that are separated temporally by the resonator round-trip time. If the input pulse width is 
much smaller than this round-trip time, individual subpulses are separated far enough that there is hardly any overlap among them. In this case, the subpulse shape does not dependent on pulse's carrier frequency. This feature is also understandable in the frequency domain. The input pulse spectrum is filtered by a comb-like transfer function of the resonator. If the pulse is short enough, its spectrum covers several peaks of this comb. In this situation, relative location of the carrier frequency with respect to cavity resonances has little effect on the output pulse shape.

\subsection{Propagation of long optical pulses}

In this section we consider a more practical situation where input pulses are considerably wider than the resonator round-trip time. Different from the short-pulse case, such a long pulse cannot be fully confined within the resonator, and only a part of the pulse that is inside the resonator experiences the refractive index change. Moreover, some parts of the pulse only experience a portion of the index change. As a result, the scaling factor $s$ and the effective time $T_{e m}$ appearing in Eq. (5.8) now themselves become time-dependent. In this situation, it is not possible to obtain an analytic expression for the output field similar to that obtained in Eq. (5.10) for short pulses.

To investigate AWC in dynamic resonators, numerical calculations are performed for a 10- $\mu$ m-long FP resonator using the time transformation method together with the linear system theory. The input pulse is still considered to be Gaussian but its 
width $T_{0}=10 \mathrm{ps}$ is much larger than the round-trip time of $0.23 \mathrm{ps}$. The facet reflectivity is increased to $98.2 \%$ to ensure a relatively large value of photon lifetime (6.5 ps). The input frequency of $192.86 \mathrm{THz}$ is again chosen to be on resonance with a cavity mode. To make the numerical results more relevant to experiments, the refractive index is decreased by only $0.1 \%$ from its initial value of 3.5 . Moreover, this change of the refractive index is not assumed to be instantaneous but is decreased linearly over a time interval of 3-6 ps (the origin of time is at the peak of the input pulse).

Transmitted pulse shape and spectrum are plotted in Fig. 5.4(a) and (b), respectively (red solid curves). The case of a static resonator is shown by dotted blue curves; dashed green curves show the input pulse for comparison. A comparison of the blue and red curves reveals the impact of $0.1 \%$ index change on the output pulse. Consider the spectral changes first. As depicted in part (b), the output spectrum shows a new peak shifted toward the blue side. This represents the AWC expected because the resonant frequencies of the cavity modes shift toward the blue side by about 190 GHZ after the index change has been completed. Indeed, the central frequency of this shifted peak agrees well with the new position of the resonator mode. The amplitude of the shifted peak is larger than that at the original frequency, indicating that a large portion of the pulse energy has shifted to the new resonator mode. The reason that some energy is still left at the original resonator mode is related to the fact that the entire pulse does not experience the index change. A portion of the front 
part of the pulse leaves the resonator before the refractive index begins to change. Similarly, a portion of the back part of the pulse enters the medium after the index change has occurred. Since this part is no longer in resonance with the shifted cavity mode, its coupling into the resonator is reduced.
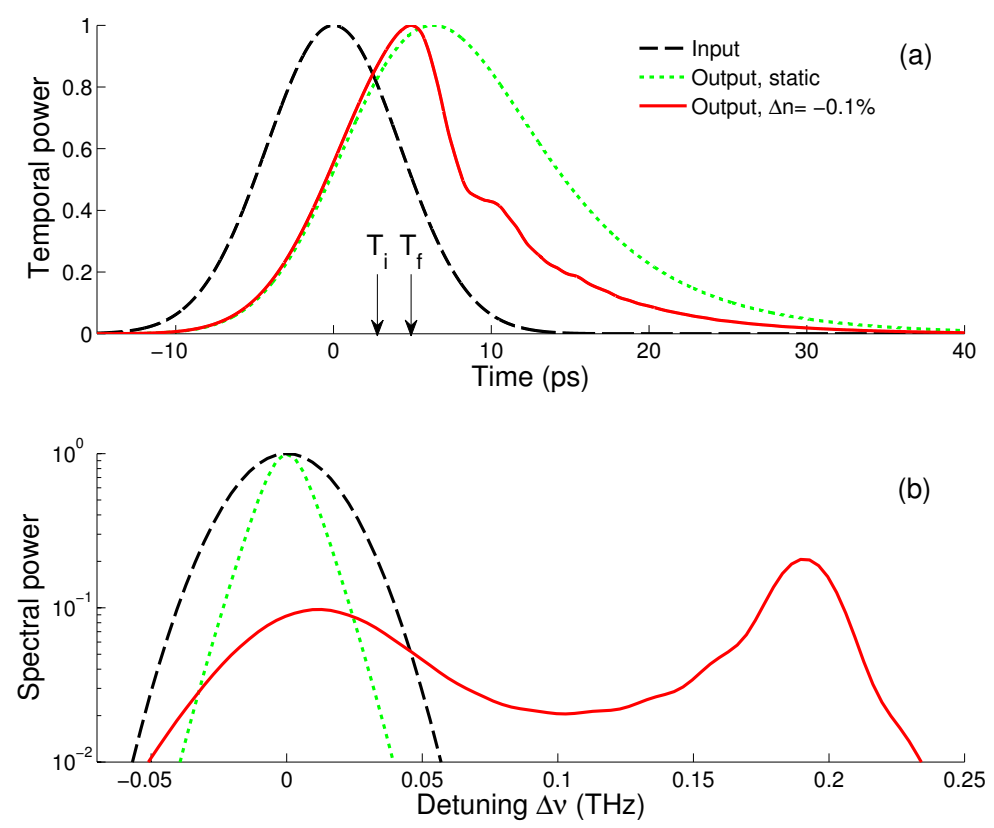

Figure 5.4: Long-pulse (10 ps) propagation for a linear change in refractive index of $-0.1 \%$ (red) between $T_{i}=3 \mathrm{ps}$ and $T_{f}=6 \mathrm{ps}$ (marked by arrows). The round-trip time of the resonator is $0.23 \mathrm{ps}$. (a) The index change advances the output peak and produces a long tail with knee-like feature. (b) The output spectrum shows two spectral peaks corresponding to the original and shifted cavity modes, respectively.

Note that some pulse energy also lies in between the original and shifted resonator modes. This energy comes from the part of the pulse that only "sees" a fraction of the 
index change (i.e., parts of the pulse that either leave or enter the resonator during the index change). This time-dependence of the output spectrum is better reflected in the spectrogram shown in Fig. 5.5, where the pulse spectrum appears to follow temporal changes in the refractive index.

Figure 5.4(a) reveals significant changes in the output pulse shape introduced by the dynamic index changes taking place inside the FP resonator. The peak of the output pulse (solid red curve) is shifted towards the front of the pulse when compared to the static case (dotted blue curve). This shift occurs because index changes shift the mode frequencies of the resonator, which makes the trailing part of the pulse off-resonance. This explains a considerable reduction in the energy contained in the trailing part the output pulse. In addition, an evidence of oscillations (period about 5 ps) of decreasing amplitude just after the index change has been completed (kneelike feature) is also seen. These oscillations could be interpreted to result from beating of light at the shifted frequency with light at the original frequency. Indeed, longer pulses show such oscillations even more clearly.

\subsection{Variations of external parameters}

The results presented in Section 5.3 show that dynamic changes in the refractive index of a $\mathrm{FP}$ resonator produce AWC during transmission of an optical pulse but they also lead to several other temporal and spectral changes in the output pulse. 


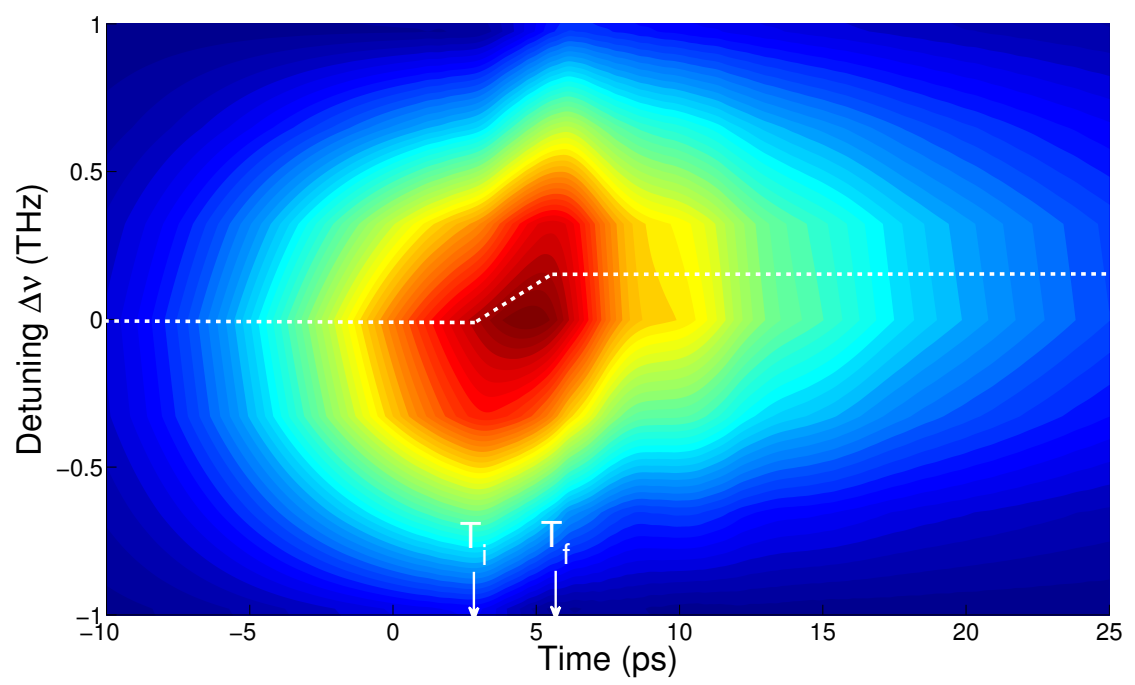

Figure 5.5: Spectrogram for the situation shown in Fig. 5.4. The pulse spectrum appears to follow the cavity resonance indicated by the dotted line.

In the time domain, the pulse becomes asymmetric and its width may increase or decrease depending on the direction in which refractive index is changed. In the spectral domain, the output spectrum develops a multi-peak structure and is distorted considerably in an asymmetric fashion. In addition, the pulse develops a considerable frequency chirp. The magnitude of these changes depends considerably on various factors that can be controlled during an experiment. In this section, the impact of three such factors related to the temporal duration over which index change is completed, the magnitude and direction of index change, and the detuning of input wavelength from the nearest FP-resonance frequency are explored. 


\subsubsection{Speed of index change}

The adiabatic nature of the AWC process requires that changes in the refractive occur on a time scale shorter than the photon lifetime of the resonator. If the index is changed slowly over a long duration, not much pulse energy is likely to remain in the resonator after the index change has been completed. The important question is how much the shape and spectrum of the output pulse depend on the speed of index change. To answer this question, the transmission of 10-ps Gaussian pulses through the same FP resonator used for Fig. 5.4 is considered again but allowing the temporal duration, $\Delta T=T_{f}-T_{i}$, over which index is reduced linearly by $0.1 \%$ to vary from 3 to 27 ps. Figure 5.6 shows the shapes and spectra of output pulses for three choices of $\Delta T$ while maintaining all other parameters (pulse width, magnitude of index change, etc.) to their same values used in Fig. 5.4. In all cases, the photon lifetime is fixed at 6.5 ps. Changes in the pulse shape are easily understood once we interpret the spectral modifications correctly. So, let us first focus on the spectra.

When the refractive index change is completed well within the photon lifetime $\left(T_{f}-T_{i}=3 \mathrm{ps}\right.$, dashed-red curve), the new AWC peak dominates the pulse spectrum as its amplitude is larger than that of the peak at the original mode frequency. However, as the duration of index change becomes larger than the photon lifetime $\left(T_{f}-T_{i}=13 \mathrm{ps}\right.$, dot-dashed black curve), the amplitude of the AWC peak is reduced

considerably. Much more pulse energy remains at the original mode frequency, and considerable pulse energy lies in between the two modes. When the duration of index 

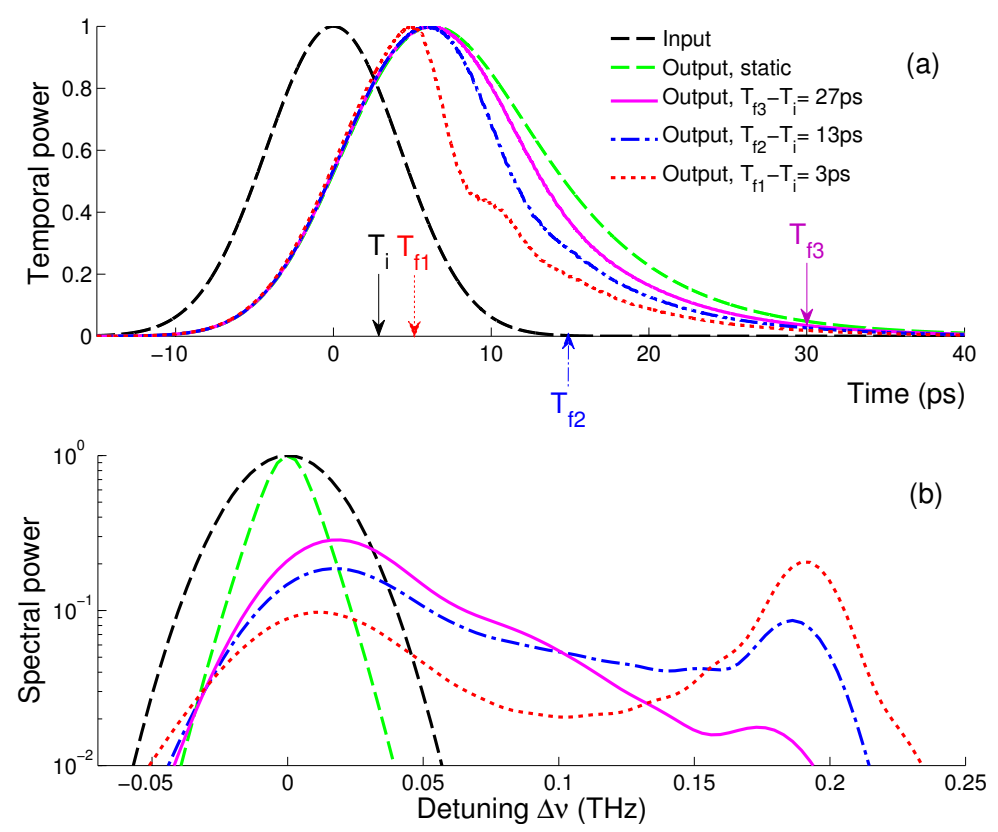

Figure 5.6: Impact of index-change duration $\Delta T=T_{f}-T_{i}$ on propagation of 10 -ps Gaussian pulses in the case of a $-0.1 \%$ linear change in the refractive index beginning at $T_{i}=3$ ps. (a) Output pulse narrows down and exhibits oscillations as $\Delta T$ decreases. (b) Amplitude of the AWC peak also increases as $\Delta T$ decreases.

change is increased further to 27 ps (solid purple curve), the AWC almost disappears as little energy of the pulse appears at the shifted mode frequency. Note, however, that the pulse spectrum is distorted considerably even in this case: It is broader and highly asymmetric compared to the static case. The reason is that spectral broadening occurs only towards the blue side. This is expected when the refractive index is reduced from its original vale and cavity modes experience a blue shift.

The efficiency of the AWC process can be characterized by a parameter $f_{s}$ rep- 
resenting the amplitude of the AWC peak relative to the input peak. Physically, $f_{s}$ represents the fraction of pulse energy that is contained within the AWC peak. This quantity is plotted by the solid blue curve in Fig. 5.7 as a function of the duration $\Delta T$ over which index changes are completed. The value $\Delta T=0$ corresponds to an instantaneous change discussed in Section 5.2. Note that $f_{s}$ increases slightly in the range of $\Delta T=0$ to $3 \mathrm{ps}$, where it reaches its peak value of about $21 \%$. Beyond that, $f_{s}$ displays a monotonic decay as $\Delta T$ increases further. The initial increase at up to $\Delta T=3$ ps results from a relatively long duration of the input pulse. When the refractive index begins to change, the energy is still being injected into the resonator, and the energy inside the resonator reaches its maximum just before the index change is completed for $\Delta T=3 \mathrm{ps}$, resulting in an optimum situation. The monotonic decay beyond that is characterized by the continuously decreasing fraction of pulse energy that enters the resonator after the index changes have been completed. Also shown in Fig. 5.7 are the $f_{s}$ for two other resonators with different values of photon lifetime: 3.4 and $12.6 \mathrm{ps}$ for the dotted black and dashed red curves respectively. Note the three resonators shown in Fig. 5.7 are identical, expect for their mirror reflectivity (96.5\%, 98.2\% and 99\% for black, blue and red cases).

\subsubsection{Magnitude of index change}

The final refractive index could be written as $n_{2}=n_{1}(1-\Delta)$, where $\Delta$ represents the magnitude of index change (relative to $n_{1}$ ). This subsection considers the impact of 


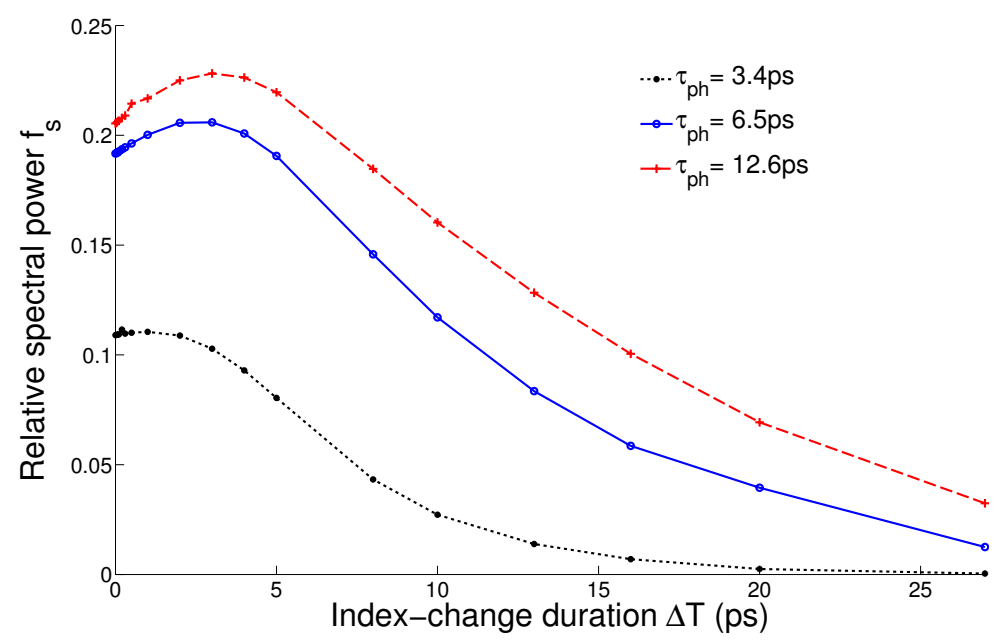

Figure 5.7: AWC efficiency as a function of index-change duration $\Delta T$ for three values of photon lifetimes $\tau_{p h}$ in the case of $\Delta n=-0.1 \%$. The efficiency is maximum for $\Delta T$ near zero when $\tau_{p h}$ is relatively small but it peaks at a value of $\Delta T>0$ that increases with $\tau_{p h}$.

different $\Delta$ on the transmitted pulse shape and spectrum. In Figs. 5.4 to 5.7, a fixed value of $\Delta=0.1 \%$ is used in the simulation. Figure 5.8 shows the shapes and spectra of output pulses by changing this value in the range of 0.05 to $0.4 \%$ while keeping all other parameters identical to those used for Fig. 5.4. In particular $T_{f}-T_{i}=3$ ps in all cases. Pulse shapes in part (a) are affected much less by changes in $\Delta$ compared with the spectral changes. As seen in Fig. 5.4(b), the AWC-induced spectral shift increases from $95 \mathrm{GHz}$ to $760 \mathrm{GHz}$ as $\Delta$ increases from $0.05 \%$ to $0.4 \%$. The amplitude of the AWC peak does not depend on $\Delta$ much and it appears to saturate for $\Delta>0.2 \%$. The larger amplitude of this peak for $\Delta=0.05 \%$ results from an overlapping of the original and shifted peaks for such small index changes. 

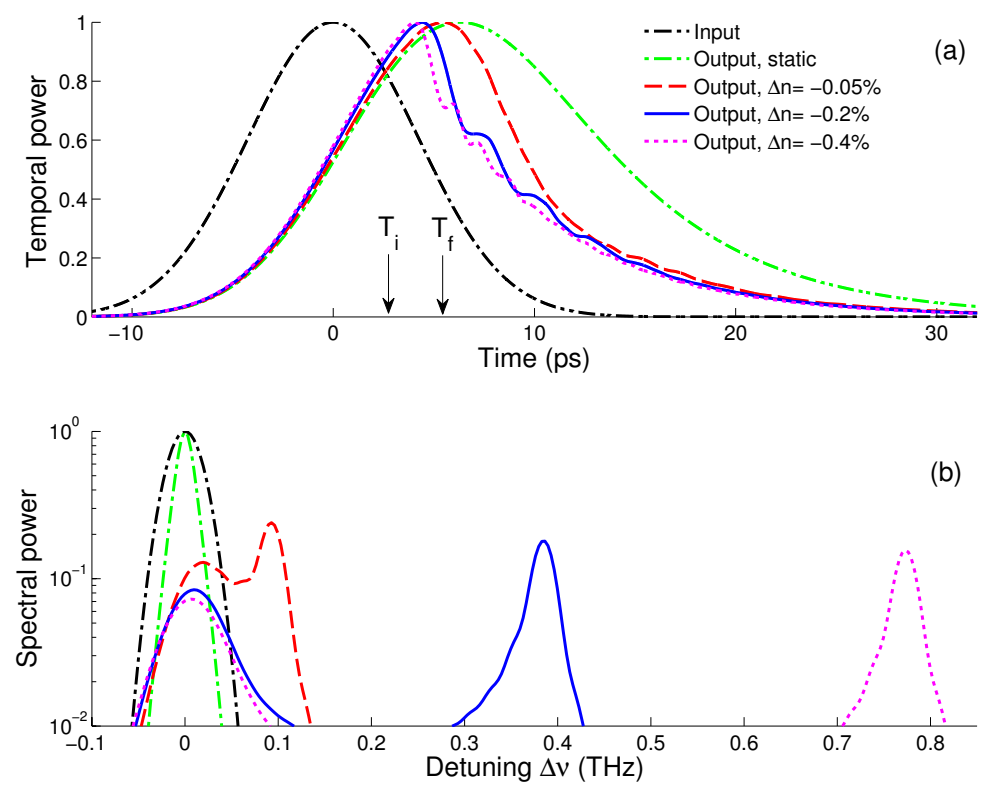

Figure 5.8: Impact of the magnitude of $\Delta n$ for a fixed linear index-change duration of $3 \mathrm{ps}$, input pulse width of $10 \mathrm{ps}$, and a round-trip time of $0.23 \mathrm{ps}$.

(a) The oscillation frequency in the pulse tail increases for a larger index change because of (b) a larger AWC-induced shift in the mode frequency.

As far as changes in the pulse shape are concerned, it can be seen from Fig. 5.4(a) that pulse peak shifts towards the front side more and more as $\Delta$ increases. As already discussed, this shift is a consequence of the trailing part of the pulse falling out of resonance after the index change has occurred. Notice, also an increase in the frequency of oscillations in the trailing part of the pulse as $\Delta$ increases. This is understandable by recalling that the oscillation frequency is equal to the spectral shift induced by the AWC process that increases with $\Delta$ rapidly as seen in Fig. 5.4(b). For $\Delta=0.2 \%$ one can see for 4 oscillation cycles with a period of about $2.5 \mathrm{ps,} \mathrm{which}$ 
agrees with a spectral shift of $0.4 \mathrm{THz}$. The oscillation period is reduced to near $1.2 \mathrm{ps}$ for $\Delta=0.4 \%$ because of a doubling of the AWC-induced frequency shift. The number of oscillations is set by the time interval over which the resonator contains light at both the original and shifted mode frequencies. In the present situation, almost all of of pulse energy within the resonator shifts to the new mode frequency after 4 cycles.

\subsubsection{Detuning of input frequency}

So far it is assumed that the carrier frequency (or the launch wavelength) of the input pulse coincides with one of the modes of the resonator. This was also the situation in most experiments, and it is often argued that on-resonance pulse launch is required for AWC to occur. This subsection considers off-resonance launching of input pulses. Figure 5.9 shows the shape and the spectrum of output pulses when the 10-ps Gaussian pulse is detuned from resonance by $\pm 25 \mathrm{GHz}$. All other parameters are kept identical to those in Fig. 5.4. In particular, $\Delta=0.001$ and $T_{f}-T_{i}=3$ ps. It is evident from Fig. 5.9 that all qualitative spectral and temporal features that occur when the input pulse is on-resonance also occur in the off-resonance case with minor modifications.

A new feature that is apparent in Fig. 5.9(a) is related to the direction in which the peak of the output pulse moves in a dynamic resonator compared to the static case (black curve). More specifically, pulse peak shifts toward left or right side depending on whether detuning is $-25 \mathrm{GHz}$ or $+25 \mathrm{GHz}$. The reason can be understood 

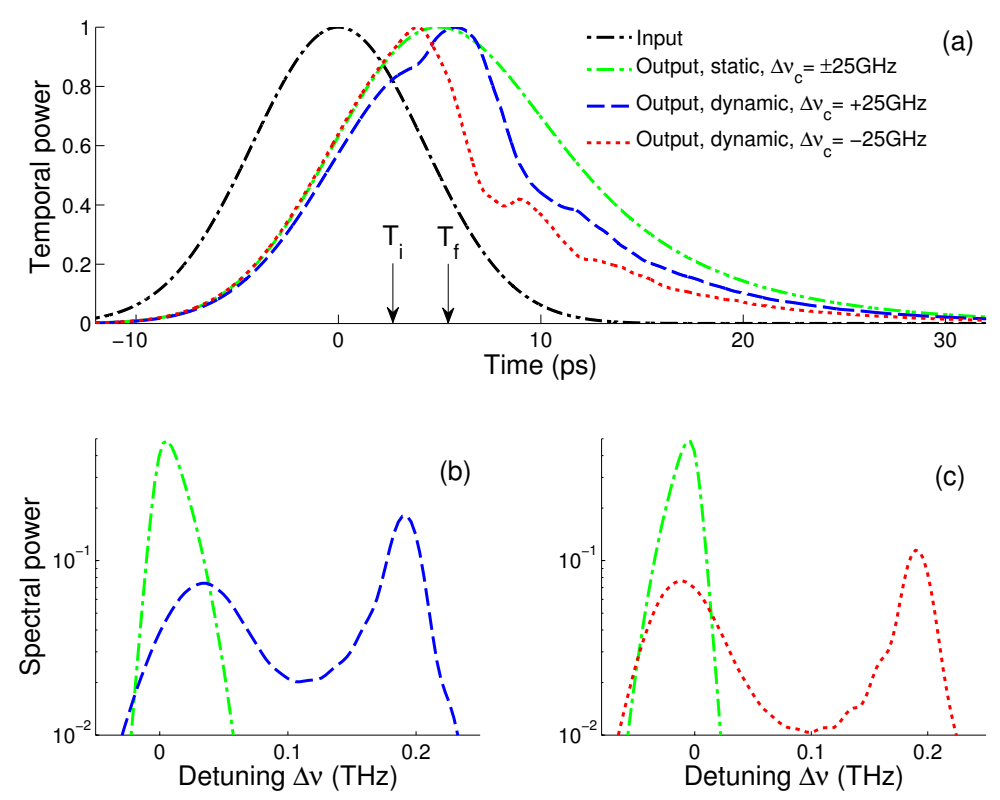

Figure 5.9: Impact of detuning $\Delta \nu_{c}$ of input pulse from a cavity resonance $\left(\Delta \nu_{c}=\nu_{0}-\nu_{c}\right.$, where $\nu_{0}$ and $\nu_{c}$ are the input carrier frequency and cavityresonance frequency, respectively) for a $-0.1 \%$ linear change in the refractive index between $T_{i}=3 \mathrm{ps}$ and $T_{f}=6 \mathrm{ps}$ (round-trip time is $0.23 \mathrm{ps}$ ). (a) Output pulse shapes show an asymmetry with respect to the the sign of $\Delta \nu_{c}$. (b) The amplitude of the AWC peak is larger for a $+25 \mathrm{GHz}$ detuning than for the $-25 \mathrm{GHz}$ detuning (in this negative index-change case).

by noting that the cavity resonance moves toward the blue side in both case (because of a negative index change). If the pulse is initially detuned on the red side of the resonance peak, a larger portion of its trailing part will experience reduced transmittance compared to when the pulse is initially detuned on the blue side. Oscillation frequency is different in the two cases because the beating frequency between the 


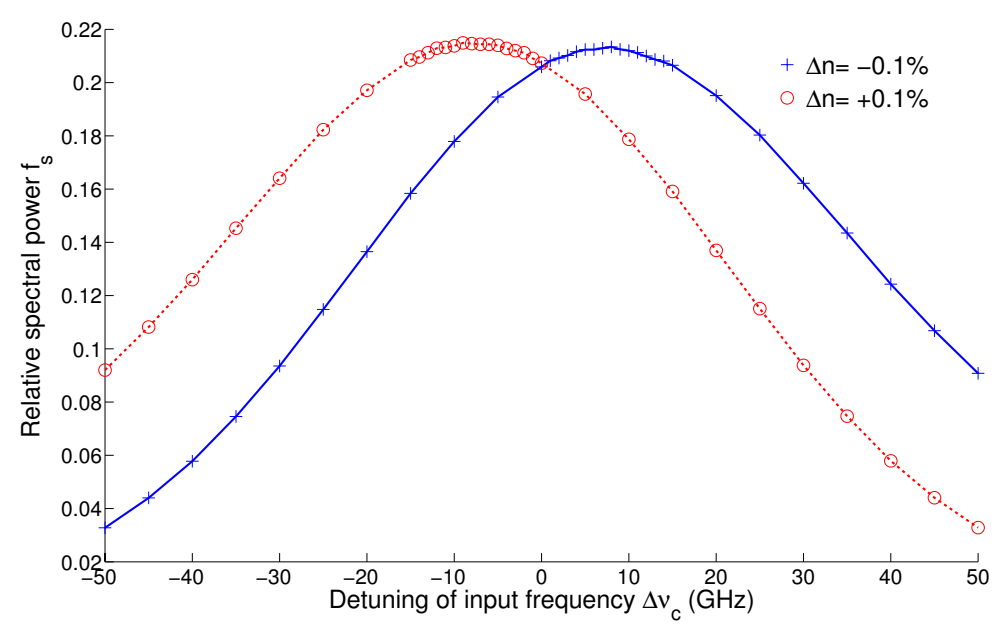

Figure 5.10: Fraction of pulse energy transferred to the AWC peak as a function of detuning of input pulse from a cavity resonance under the same long-pulse conditions as in Fig. 10 but for an index change of $-0.1 \%$ (blue) and $+0.1 \%$ (red).

original and shifted peaks are different (about 140 and $200 \mathrm{GHz}$ ) as seen from the spectra in parts (b) and (c).

The relative amplitude of the AWC peak is larger in the case of positive detuning (case b in Fig. 5.9) compared to the case of negative detuning (case c). This can also be understood from the blue shift of the AWC peak in the case of a negative index change. The opposite would happen if the index was increased and the AWC peak was shifted toward the red side of the original peak. This behavior is shown more clearly in Fig. 5.10 by plotting the relative amplitude $f_{s}$ of the AWC peak as a function of input detuning. A somewhat surprising feature of this figure is that, contrary to the conventional wisdom, the maximum energy transfer to the AWC peak 
does not occur under on-resonance conditions. Clearly, if the objective is to transfer as much pulse pulse energy as possible to the AWC peak, one must detune the input pulse in the same direction in which the resonator mode is expected to shift after changes in the refractive index have been completed.

In summary, this Chapter presents a simple and intuitive model based on the impulse response of linear systems for describing the propagation of optical pulses through a dynamic Fabry-Perot resonator, containing a linear medium whose refractive index changes with time. It shows that the adiabatic wavelength conversion results from a scaling of the slice transit time by a factor that depends on both the magnitude and sign of index change. The time transformation method is first applied to the case in which pulses short compared with the round-trip time are transmitted, and the refractive index of the resonator is changed instantaneously after a pulse has been launched. The case of pulses longer than the round-trip time is discussed in detail as it is expected to be more relevant experimentally. We consider transmission of picosecond Gaussian pulses through a 10- $\mu$ m-long FP resonator and allow the refractive index of the resonator to change over a duration ranging from 3 to 30 ps. The magnitude of index change also varies between 0 to $0.4 \%$. It is shown that an AWC process is always accompanied by significant changes in both the shape and the spectrum of output pulses. How such temporal and spectral changes depend on externally controllable parameters such as the magnitude and the speed of index change and detuning of the input pulse from the cavity resonance, is discussed in detail. 


\section{Kerr Media: Instantaneous}

\section{Nonlinearity}

In the previous two chapters, we showed that the proposed time transformation method could be applied to the dynamic linear media and to explain the phenomenon of adiabatic wavelength conversion (AWC) in a simple fashion. As is discussed in this chapter, even more interesting physics happens when optical pulses propagate through nonlinear media. The second part of this thesis is devoted to the application of the time transformation method to the nonlinear media.

Specifically, the time transformation method is first applied in this chapter to a Kerr medium, whose response is assumed to be instantaneous. Unlike the case of dynamic linear media, it is shown that both the scaling factor as well as the temporal mapping become nonlinear. Numerical examples are provided to show that time transformation method agrees with nonlinear Schrödinger (NLS) equation for relatively long pulses and pulses containing a few optical cycles. In the single-cycle

region where the NLS equation may fail because of the underlying slowly varying 
envelop approximation (SVEA), we show that the time transformation method agrees with the the finite-difference time-domain (FDTD) method that integrates Maxwell's equations directly.

\subsection{Time transformation by a Kerr medium}

As is discussed in Chapter 3, pulse propagation through a dynamic optical medium can be described as a temporal mapping of input electric field slices located at $t^{\prime}$ to corresponding output slices located at $t$ via:

$$
t=t^{\prime}+T_{d}\left(t^{\prime}\right)
$$

where $T_{d}\left(t^{\prime}\right)$ is the transit time associated with the slice of the input pulse at time $t^{\prime}$. The temporal mapping in Eq. (6.1) is referred to as the time transformation. In addition to a shift of the temporal location through Eq. (6.1), time transformation also modifies the amplitude of a temporal slice according to

$$
E_{\text {out }}(t) d t=E_{\text {in }}\left(t^{\prime}\right) d t^{\prime}
$$

As shown in Chapters 4 and 5, this idea can be applied to a dynamic linear medium to describe the process of adiabatic wavelength conversion, a process that occurs when the medium's refractive index changes with time [1]. One might ask: can this simple method, developed from the linear system theory, be applied to nonlinear media as well? Here, we show that the time transformation method indeed can be 
applied to a nonlinear medium. More specifically, the nonlinear medium is treated as being linear, but its refractive index is allowed to change with time as dictated by the pulse intensity profile. Such an approach is similar to that used in Ref. [90] in the context of nonlinear optical waveguides.

Unlike the NLS equation that discards the rapid temporal oscillation of the electric field in favor of the slowly vary pulse envelope, the time transformation method maps directly the input electric field into the output one. As a specific nonlinear optics example, let us consider a non-dispersive Kerr medium whose refractive index

$$
n(t)=n_{0}+n_{2} I(t)
$$

is separated into a linear part $n_{0}$ and a nonlinear part that is the product of the Kerr nonlinear coefficient $n_{2}$ and the intensity $I(t)$ is related to the electric field as

$$
I(t)=\frac{n_{0} c \epsilon_{0}}{2}|E(t)|^{2}
$$

A direct consequence of such an intensity-dependent refractive index is that different electric-field slices of the pulses are transported through the medium at different speeds, $v(t)=c / n(t)$, depending on the local value of refractive index $n(t)$. In other words, the transit time of a slice depends on the local intensity $I(t)=I_{0} f(t)$ of the pulse as

$$
T_{d}\left(t^{\prime}\right)=T_{d 0}+n_{2} I_{0} f\left(t^{\prime}\right) L / c,
$$

where $I_{0}$ is the peak intensity of a pulse with the shape $f(t), L$ is the medium length, $c$ is the speed of light in vacuum, and the constant part, $T_{d 0}=n_{0} L / c$ comes from the 

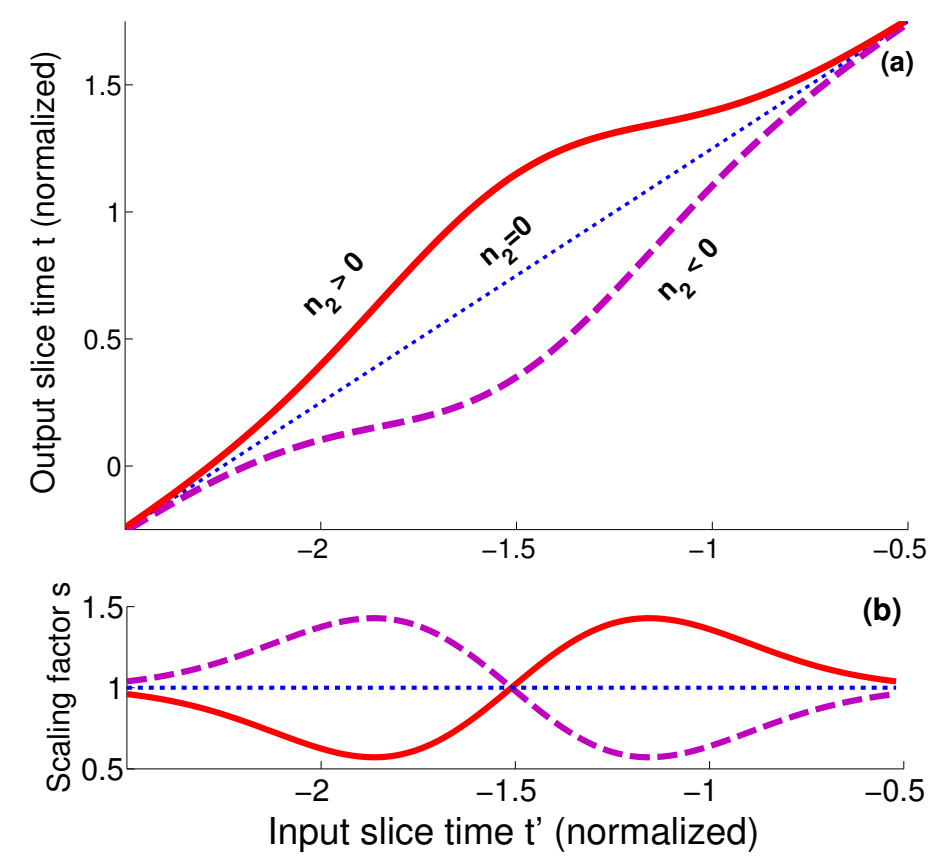

Figure 6.1: (a) Time transformation performed by a nonlinear Kerr medium for Gaussian pulse propagation and (b) the corresponding scaling factor s. Cases: $n_{2}>0$ (solid); $n_{2}<0$ (dashed); $n_{2}=0$ (dotted)

contribution of linear refractive index.

Figure 6.1(a) shows the time transformation performed by a Kerr medium for a Gaussian pulse having $\left[f(t)=\exp \left(-t^{2} / T_{0}^{2}\right)\right]$ using $T_{d 0} / T_{0}=2$ and $\tau_{n} / T_{0}=0.8$, where $\tau_{n}=n_{2} I_{0} L / c$ is the maximum nonlinear delay experienced by the pulse. The spacing of the time slices is determined by the slope of time transformation curve in Fig. 6.1(a), $d t / d t^{\prime}$, related inversely to the scaling factor $s$, i.e. ,

$$
s=\frac{d t^{\prime}}{d t} .
$$

In the case of a linear medium $\left(n_{2}=0\right)$, the 45 -degree slope indicates that the in- 
put and output time slice have the same duration, corresponding to a linear time transformation. In the case of a dynamic linear medium shown in Fig. 4.2, this time transformation is still linear, although its slope deviates from one. As discussed in Chapter 4, uniform time compression and time stretching happen for dynamic linear media with $s<1$ and $s>1$, respectively. However, in the case of a nonlinear medium, this time transformation becomes nonlinear because the local refractive index is different for different electric field slices. As a result, the transit time [Eq. (6.5)], as well as the scaling factor [Eq. (6.6)] vary along the pulse in a nonlinear fashion.

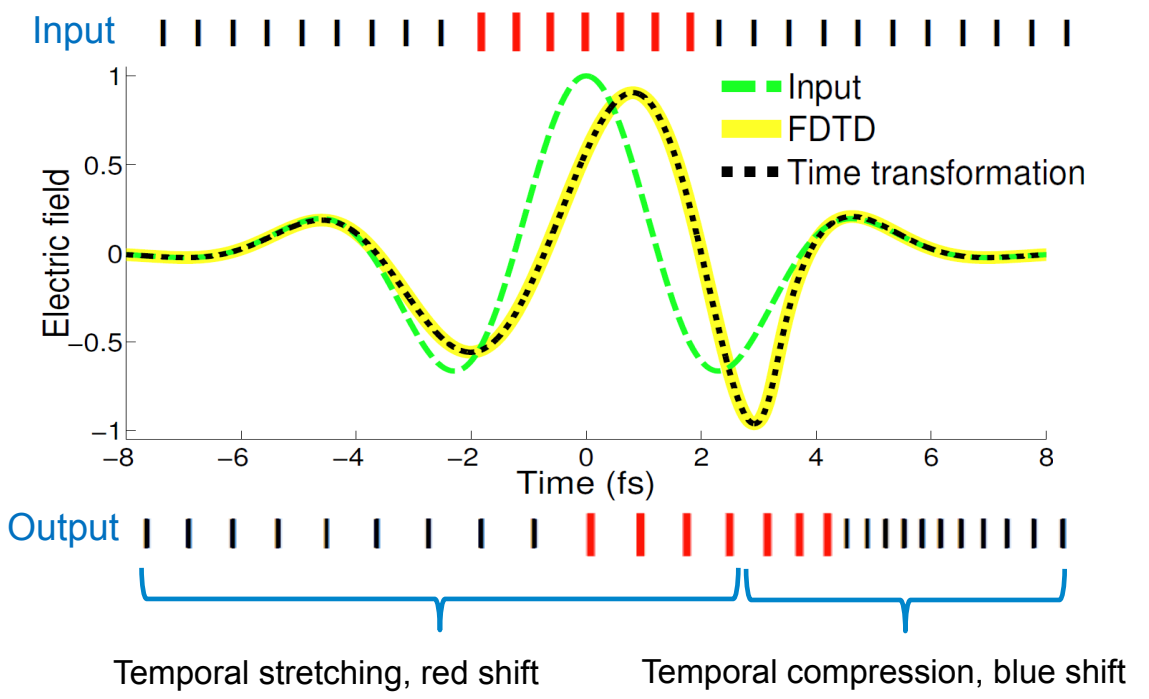

Figure 6.2: Output (dotted-black) and input (dashed-green) electric field for a single-cycle pulse after it propagates through a nonlinear Kerr medium. The predictions from FDTD calculation is also plotted for comparison using solidyellow curve. Two approaches agree with each other quite well.

In order to see the impact of such a nonlinear time transformation on an optical 
pulse, Fig. 6.2 plots the input and transmitted electric field for a single-cycle pulse that propagates through a nonlinear Kerr medium with $n_{2}>0$. The top bar shows the input slices, which are uniformly spaced with the red ones showing slices near the pulse center. The bottom bar shows the output slices, which are no longer uniformly spaced because the speed of light $c / n$ is different for different parts. More specifically, slices near the pulse center experience higher values of light intensity, which results in a slower travel speed. We can see these slices (marked by red color) move towards the trailing part of the pulse, leading to the distortion of the pulse shape. This is the physical origin of self-steepening [60] in the time transformation picture.

Meanwhile, as a result of such a nonlinear temporal mapping, the spacing between adjacent slices increases in the front part of the pulse, while it decreases in the trailing part of the pulse, as shown by the bottom bar in Fig. 6.2. Notice that the total number of slices does not change during this temporal mapping process. As a consequence, the front part of the pulse experiences temporal stretching, causing the red shift of the carrier frequency for this portion of the pulse. On the other hand, temporal compression happens at the trailing part, leading to the blue shift of the spectrum. This variation in the optical frequency is the origin of SPM [58] in the time transformation picture. Creation of the new frequency components (frequency chirping) is a simple consequence of the nonlinear temporal stretching or compression of the electric field slices during the temporal mapping. 


\subsection{Non-dispersive Kerr medium}

To test the validity of the time transformation method on nonlinear problems, the propagation of a Gaussian input pulse through a non-dispersive Kerr medium is considered in this section. More specifically, the input pulse width is varied and the results are compared with those obtained from the NLS equation and the FDTD technique for relatively long pulses and single-cycle pulses, respectively.

\subsubsection{Relatively long pulses: self-phase modulation}

This subsection considers the propagation of relatively long pulses. Here the words "relatively long" mean that there are many optical cycles within the envelope of the pulse so that the self-steepening factor $1 /\left(\omega_{0} T_{0}\right) \ll 1$ [59], i.e., self-steepening has negligible impact on the pulse propagation. In Fig. 6.3 (a) and (b), the input and output shapes and spectra are compared for the propagation of a 10-ps Gaussian pulse $\left(f_{0}=\omega_{0} / 2 \pi=200 \mathrm{THz}\right)$ through a Kerr medium. The pulse has a peak intensity such that $\phi_{m}=2 \pi f_{0} \tau_{n}=20$, where $\phi_{m}$ is the maximum SPM-induced phase shift [59]. As shown in part (a), the pulse shape does not change much (the input pulse shape falls under the plotted output shape). However, the output spectrum in part (b) shows considerable SPM-induced broadening.

The results from our time transformation method (solid-yellow) match the predictions from the standard approach based on the NLS equation (dotted-black), which 
takes the following form in the absence of dispersive effects:

$$
\frac{\partial A}{\partial z}=i \gamma|A|^{2} A
$$

where $A(z, t)$ is the envelope of the pulse and $\gamma=n_{2} \omega_{0} / c$ is a nonlinear parameter [59]. It is clear from Eq. (6.7) that a relatively long pulse only experiences a nonlinear phase shift, and nothing happens to its temporal pulse shape as it propagates through a nonlinear Kerr medium. In the time transformation picture, the nonlinear temporal relocation of the electric field slices does happen for long pulses. But the nonlinear time shift for the slices is negligible to the pulse width, i.e., $\tau_{n} \ll T_{0}$, and hence can hardly be seen in the temporal domain. However, these "tiny" nonlinear shift of the electric field slices can be seen in the spectral domain. Combined with the fast oscillation from carrier frequency $\omega_{0}$, one indeed "see" considerable spectral broadening (solid-yellow curve) in Fig. 6.3(b).

\subsubsection{Relatively short pulses: self-steepening}

The direct application of the time transformation method, however, assumes that the pulse shape does not change considerably during its propagation inside the medium. This requirement can be understood from Eq. (6.5), where in writing the expression

for the transit time, a constant shape $f\left(t^{\prime}\right)$ that does not change with propagation is assumed for a input slice locating at $t^{\prime}$. The temporal shape remains unchanged and this requirement is fulfilled automatically for relatively long pulses. However, such an assumption is not necessarily true for short pulses whose shape might change during 

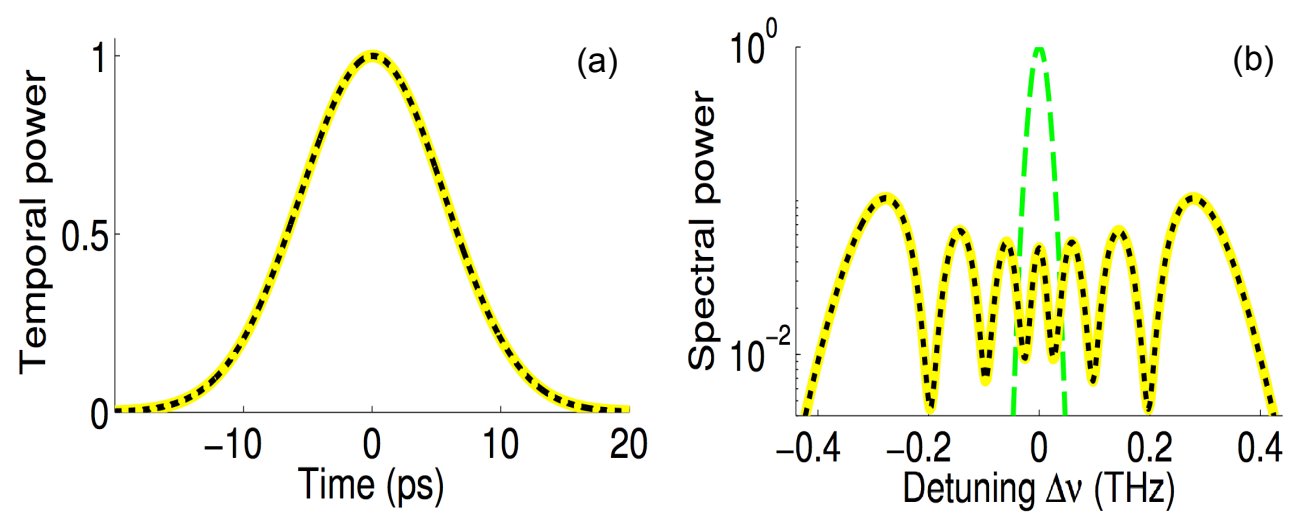

Figure 6.3: Output shape and spectrum of a 10-ps Gaussian pulse obtained with the time transformation method (solid lines) and using the NLS equation (dotted lines). Dashed curve shows input spectrum.

propagation because of self-steepening resulting from an intensity-dependent group velocity.

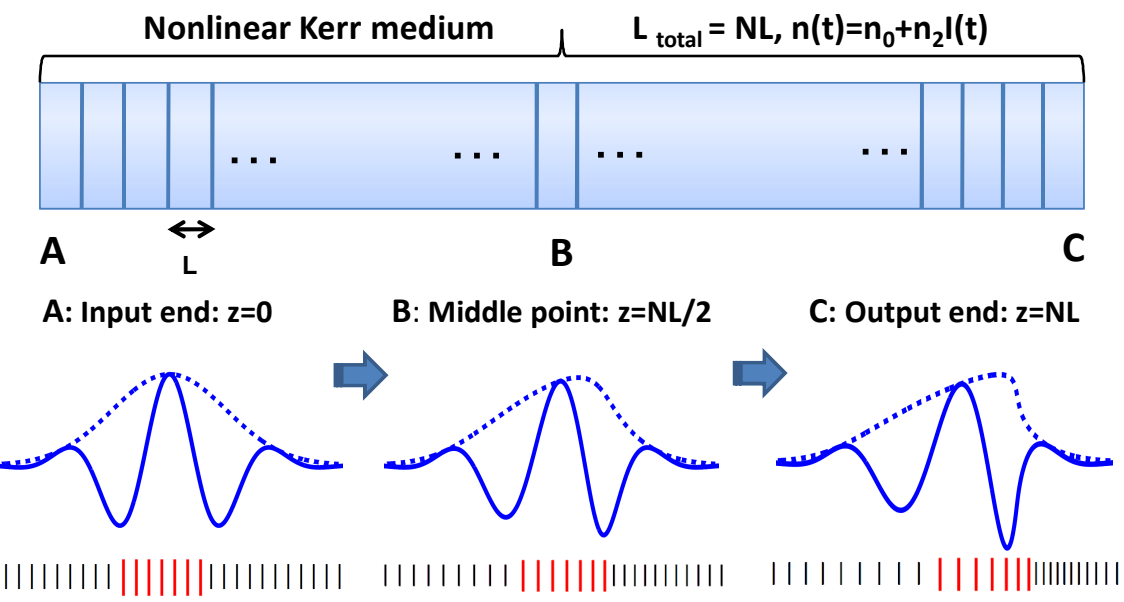

Figure 6.4: Schematic propagation of the electric field of an optical pulse through a Kerr medium $\left(n_{2}>0\right)$. Dotted curves show pulse envelope. Red time slices at the bottom move toward the back of the pulse. 
As shown in Fig. 6.4, to account for shape changes, the whole medium is divided into small sections and time transformation is applied in a step-wise fashion from one section to another, a technique similar to that used for the split-step Fourier method used commonly for nonlinear optical problems [59]. More specifically, the output pulse shape obtained at the end of one section is used as the intensity profile seen by the next section. Note that for step-wise calculations, the length $L$ in Eq. (6.5) is the length of a small section and the whole medium length $L_{\text {total }}=N L$, for a medium divided into $N$ sections.

Solid (yellow) curves in Fig. 6.5 (a) and (b) show the predicted shape and spectrum of 100-fs Gaussian pulses using the time transformation method; all other parameters are identical to those in Fig. 6.3. As seen in part (a), the pulse shape is distorted considerably inside the nonlinear medium through self-steepening. This distortion is due to the nonuniform stretching or compression of various temporal slices. The pulse spectrum in part (b) broadens through SPM as expected, but it also becomes asymmetric because of self-steepening on SPM. The number of sections in $z$ was increased until the output power profile converged; 40 sections were required for the value $\tau_{n} / T_{0}=0.2$ used in numerical simulations.

The standard approach for calculating the propagation of ultrashort pulses uses a generalized version of Eq. (6.7) that includes the self-steepening effect by adding an additional term [Eq. (2.14)]. The results of Eq. (2.14) are shown by dotted lines in Fig. 6.5 (a) and (b), and match our time transformation method, but take 20 times 

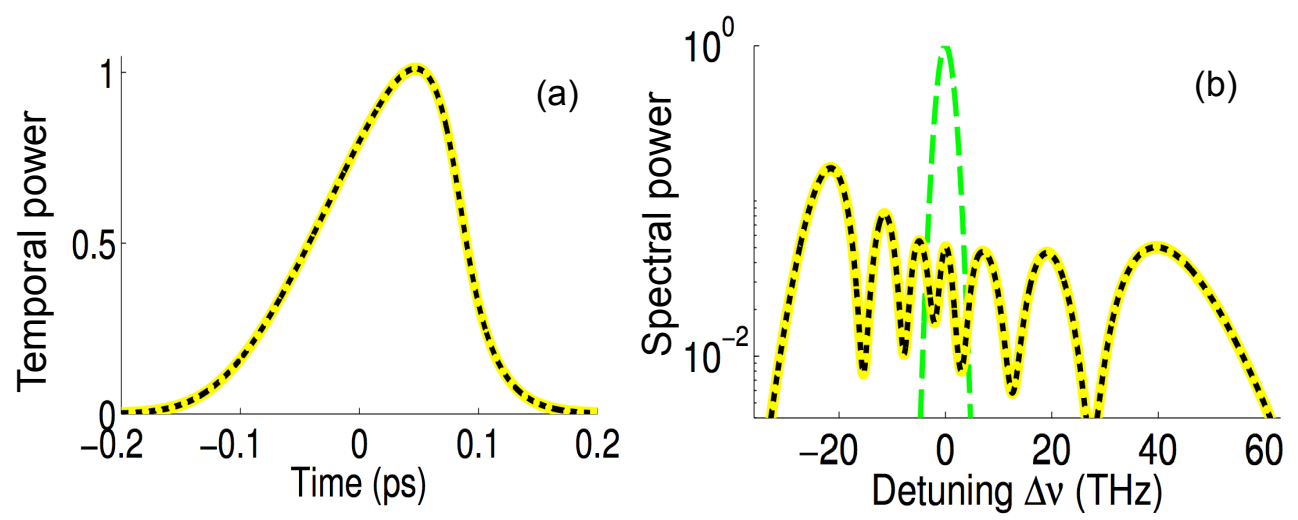

Figure 6.5: Output shape and spectrum of a 100-fs Gaussian input pulse obtained with the time transformation method (solid lines) and using the NLS equation (dotted lines). Dashed curve shows input spectrum.

longer time numerically.

\subsubsection{Few-cycle pulses}

The nonlinear propagation of few-cycle pulses is considered in this subsection. As is discussed in Section 1.2, the use of NLS equation becomes "questionable" for such short pulses because of its use of the SVEA. In this region, Maxwell's equation are solved using the FDTD algorithm. In principle, the FDTD approach can be applied to pulses of arbitrary widths because it dose not assume SVEA and makes essentially no approximation. However, FDTD requires longer and longer computation time as pulse width increases because it requires time slot $\Delta t$ to be a small fraction of $\omega_{0}^{-1}$ and step size $\Delta z$ to be a small fraction of the wavelength $\lambda$. On the other hand, the time transformation method works directly with the electric field, does not require 
the SVEA and can be used for propagation of pulses of any duration.

The solid curve in Fig. 6.2 shows the output electric field of a single-cycle Gaussian pulse $\left(5\right.$-fs, $\left.f_{0}=200 \mathrm{THz}\right)$ using the time transformation method by dividing the medium into $N=45$ sections. Note that in this case, $\phi_{m}$ is chosen to have a relative small value of 1 so that shock does not form. As shown in Fig. 6.2, the nonlinear stretching and compression of the electric field occur in the front and back part of the pulse, respectively, which directly lead to red- and blue-shift of the carrier frequency (SPM). Also, pulse peak moves towards its trailing part, leading to pulse shape distortion (self-steepening), as a result of the nonlinear temporal mapping.

The same calculation was performed using the FDTD method, and the result is plotted by the solid-yellow curve in Fig. 6.2. The excellent agreement with the FDTD method shows that the time transformation method indeed can be applied to study single-cycle pulses. What is more important is that our method is generally much faster than the FDTD approach; a factor of 50 less time was taken in the case of Fig. 6.2.

\subsection{Dispersive Kerr medium}

In Section 6.2, we considered a non-dispersive Kerr medium. In reality, however, all materials are dispersive. The impact of dispersion cannot be ignored and should be incorporated. This section considers the propagation of pulses through a nonlinear 
dispersive medium with the time transformation method. More specifically, the predictions are compared with those of the generalized NLS equation and the FDTD technique for the propagation of an optical pulse through a nonlinear dispersive Kerr medium. To simplify the following discussion, the expansion in Eq. (2.19) is used and dispersion up to second order is considered through $\beta_{2}$. In general, both the nonlinear parameter $n_{2}$ and the dispersion parameter $\beta_{2}$ can take either positive or negative values. Although the time transformation method works for all four possible sign combination of these two parameters, a medium exhibiting self-focusing nonlinearity $n_{2}>0$ with anomalous dispersion $\left(\beta_{2}<0\right)$ is used as an example because optical solitons can form in this case [59]. In the following numerical simulations the soliton order is chosen as $N=1$, a condition required for the formation of fundamental solitons that preserve their shape for a specific input pulse shape.

\subsubsection{Relatively long pulses: soliton propagation}

This subsection considers relatively long pulses containing a large number of optical cycles since the NLS equation (2.21) provides an accurate description for such pulses. More specifically, a Gaussian shape input pulse is chosen with the electric field

$$
E_{\text {in }}(t)=E_{0} e^{-t^{2} / 2 T_{0}^{2}} \cos \left(2 \pi f_{0} t\right)
$$

with $T_{0}=10 \mathrm{ps}$ at a carrier frequency of $f_{0}=200 \mathrm{THz}$. Such a pulse contains more than eight thousand optical cycles in the spectral region near $1550 \mathrm{~nm}$. 

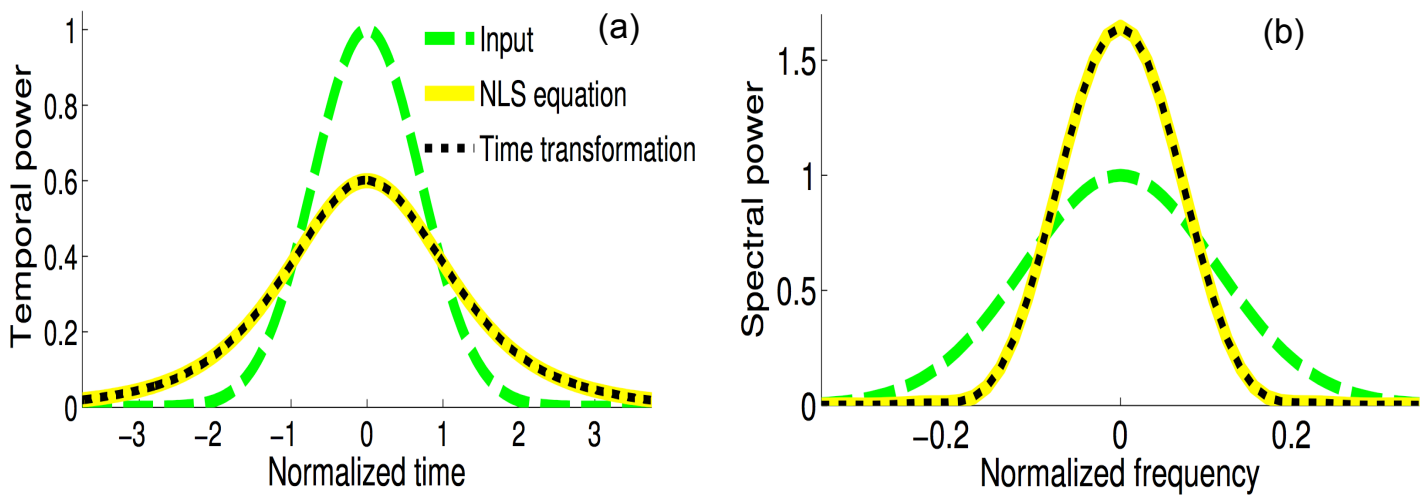

Figure 6.6: Shape (a) and spectrum (b) of a wide optical Gaussian pulse $\left(T_{0}=\right.$ $10 \mathrm{ps}$ ) at the input end (dashed green lines) and the output end at $z=4 L_{D}$ (dotted black lines). The predictions of the NLS equation are shown by solid yellow lines.

Figure 6.6 compares the predictions of our time transformation method with those of the NLS equation by plotting the pulse shape and spectrum after a distance of $z=4 L_{D}$. The input shape and spectrum are shown by the dashed green curves. Both pulse shape and spectrum change considerably inside the nonlinear medium even when the soliton condition $N=1$ is satisfied. These changes occur because the input pulse evolves to become a fundamental soliton with a 'sech' pulse shape [59]. The important feature is that both the shape and the spectrum of the pulse obtained with the time transformation method (dotted black curves) coincide perfectly with those obtained with the NLS equation (solid yellow curves), verifying that this method provides a correct description of pulse propagation inside a nonlinear dispersive medium. Although not shown in Fig. 6.6, time transformation method propagates the electric 
field, rather than the pulse envelope, and correctly includes all linear and nonlinear phase shifts.

The most well-known feature of the interplay between nonlinearity and dispersion is the fundamental optical soliton forming when the input pulse has hyperbolic secant shape with a peak intensity such that $N=1$ [59]. The existence of such a soliton is verified by replacing the electric field in Eq. (6.8) with

$$
E_{\text {in }}(t)=E_{0} \operatorname{sech}\left(t / T_{0}\right) \cos \left(2 \pi f_{0} t\right)
$$

at $z=0$. As shown in Fig. 6.7, the intensity profile of the output pulse (dashed black curve) calculated with the time transformation method overlaps perfectly with that

of the input pulse (solid yellow curve) even after propagating a distance of $10 L_{D}$. It is further verified that the fundamental soliton was able to maintain its shape even for much longer distances.

\subsubsection{Pulses containing a few optical cycles}

One important feature of the time transformation method is that it works directly with the electric field associated with an optical pulse without employing the SVEA. In principle, it is capable of describing the propagation of optical pulses of arbitrary durations. A question one may ask is whether a fundamental soliton, such as the one shown in Fig. 6.7, will maintain its shape even in the presence of self-steepening. Figure 6.8 shows the intensity profile of a 10 -fs fundamental soliton at a distance 


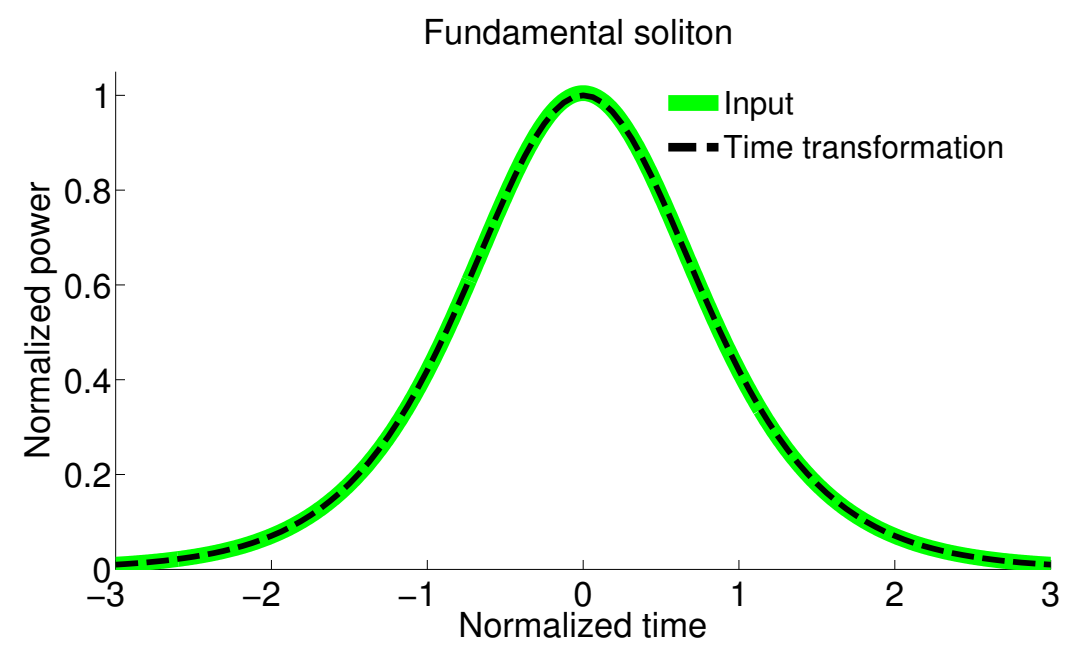

Figure 6.7: The input (solid yellow) and output (dashed black) intensity profiles at $z=0$ and $z=10 L_{D}$ when parameters of the input pulse correspond to a fundamental soliton.

of $z=10 L_{D}$ for a self-steepening factor of $1 /(4 \pi)$. Such a pulse contains about 8 optical cycles, a region where the self-steepening effect becomes important and can no longer be ignored. Numerical calculations based on time transformation method are performed for such a pulse using $N=1$.

As seen in Fig. 6.8, even though the pulse peak moves towards the trailing part because of an intensity-dependent group velocity, the shape of the soliton is almost identical to that of the input pulse, and temporal broadening can hardly be seen. Corresponding solutions of the generalized NLS (GNLS) equation are shown in Fig. 6.8 using solid yellow curves. As is apparent there, the time transformation method agrees perfectly with the predictions of GNLS equation. This is not surprising since the time transformation method includes all nonlinear effects automatically. 


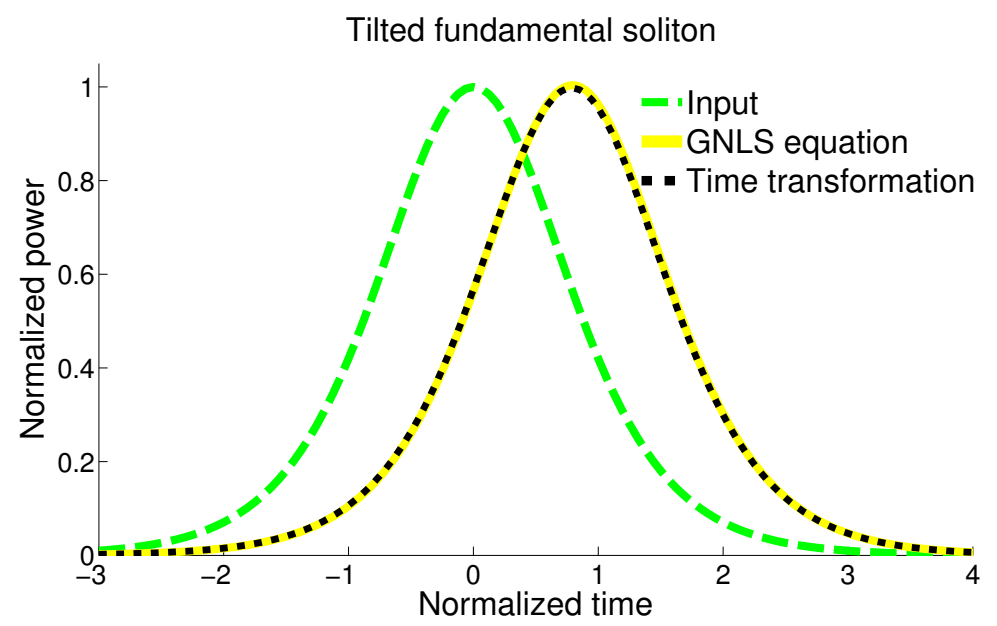

Figure 6.8: Intensity profile of a sech pulse at a distance of $z=10 L_{D}$ for parameters identical to those of Fig. 6.7 except for $T_{0}=10 \mathrm{fs}$, which results in a self-steepening factor $1 /(4 \pi)$. Input profile is depicted by the dashed-green curve. Predictions of the GNLS equation are shown by the solid yellow curve.

As discussed in Chapter 3, time transformation method can include all orders of dispersion. Here, an example of the nonlinear dispersive propagation of a higher oder soliton under the effects of third-order dispersion is shown. Specifically, the Cherenkov radiation from a third-order soliton is considered. To simulate this, a normalized third order dispersion coefficient of $\beta_{3} /\left(6 T_{0}\left|\beta_{2}\right|\right)=0.02$ was chosen, and the input pulse intensity was increased so that $N=3$. The evolution of the pulse temporal and spectral intensities for a propagation distance of $1 L_{D}$ are shown in Fig. 6.9. As shown in the left figure, starting at about $z \approx 0.4 L_{D}$, the pulse splits into two parts. The first part is soliton fission, the other part, is the dispersive wave or the Cherenkov radiation, that travels at a different group velocity than the main 

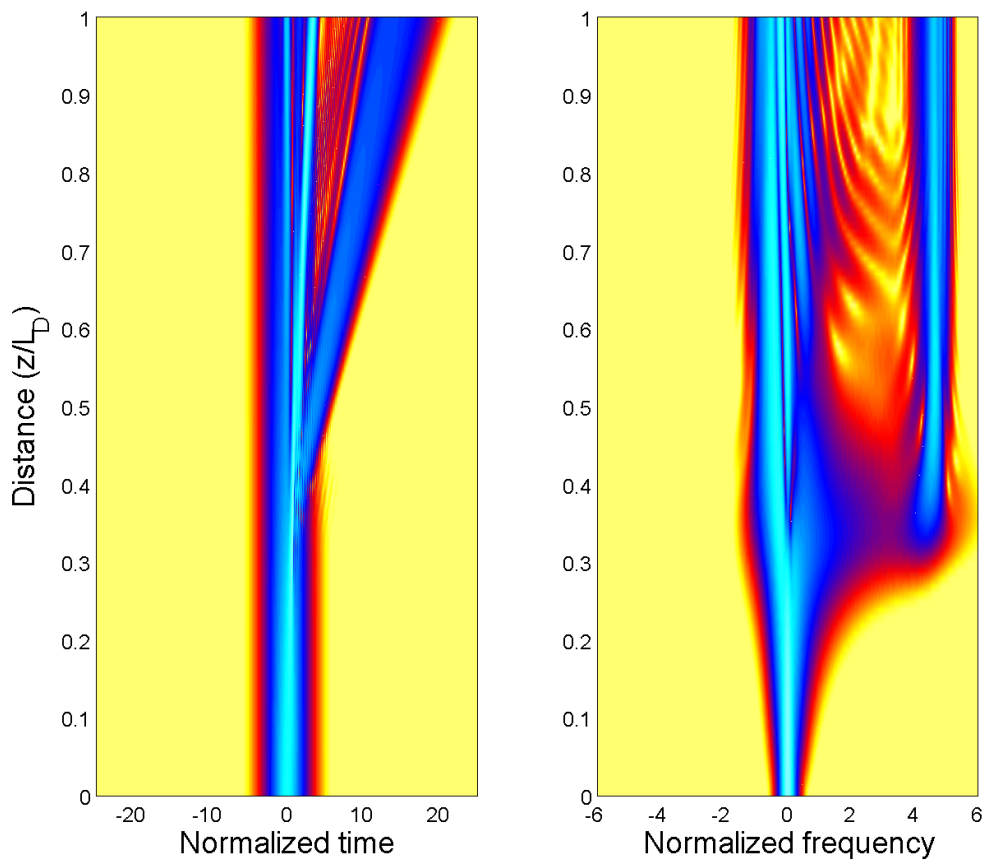

Figure 6.9: Evolution of the temporal and spectral intensity for a 10 fs thirdorder soliton $(N=3)$ propagates for a distance of $z=1 L_{D}$. The strength of the third order dispersion is chosen so that the normalized third order dispersion coefficient $\beta_{3} /\left(6 T_{0}\left|\beta_{2}\right|\right)=0.02$.

soliton. The generation of the dispersive wave, with a normalized frequency shift $\left(\nu-\nu_{0}\right) T_{0} \approx 5$, can also be clearly seen in the spectral plot on the right part of Fig. 6.9. The same calculation was also performed by solving the NLS equation and the results were identical to those shown in Fig. 6.9. 

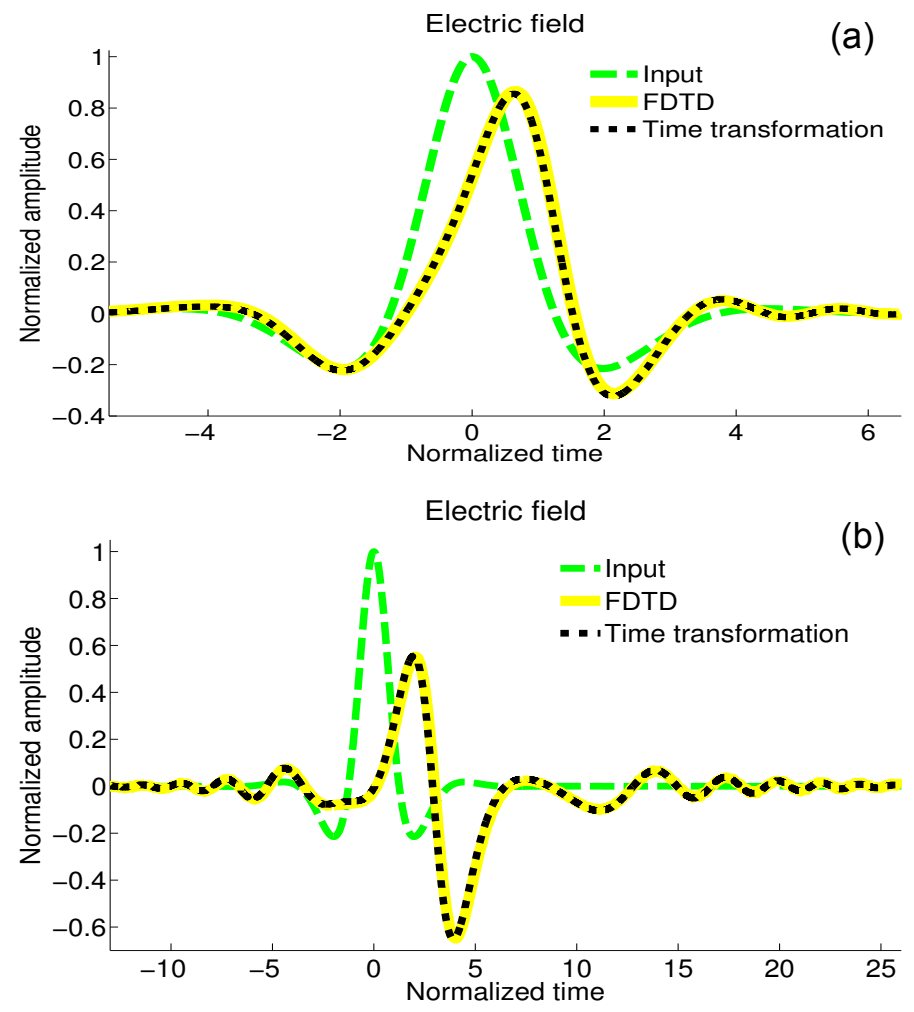

Figure 6.10: Electric field of a single-cycle pulse (dotted black curve) at $z=L_{D}$ (a) and $z=5 L_{D}$ (b) for $\omega_{0} /(2 \pi)=200 \mathrm{THz}, T_{0}=1 \mathrm{fs}$, and $N=1$. Input profile is depicted by the dashed green line. Predictions of the FDTD method are shown by the solid yellow line.

\subsubsection{Single-cycle pulses}

The case of single-cycle pulses is considered in this subsection by choosing $T_{0}=1 \mathrm{fs}$ and $\omega_{0} /(2 \pi)=200 \mathrm{THz}$. Such a pulse contains only one optical cycle. The selfsteepening parameter now has a relatively large value of about 0.8 , indicating that the electric field of the pulse will be severely distorted during its propagation. For such short pulses, envelope is not well defined and we plot the electric field. The 
results from the time transformation method are compared with those obtained by solving Maxwell's equations directly with the FDTD method. In both cases, the input electric field is taken to be

$$
E_{\text {in }}(t)=E_{0} \operatorname{sech}\left(t / T_{0}\right) \cos \left(2 \pi f_{0} t\right)
$$

with $E_{0}$ chosen to ensure $N=1$. Figure 6.10 (a) shows changes in the electric field occurring after one dispersion length. As expected, the electric field is distorted considerably when compared to that at the input. The FDTD result shown by the solid yellow curve indicates that time transformation method works well in the singlecycle regime and provides results numerically much faster than the FDTD technique.

As a further check, Fig 6.10 (b) shows the electric field after the single-cycle pulse has propagated a distance of $5 L_{D}$. As seen there, compared to the input pulse, the electric field has spread over a much larger temporal range, and its central peak has moved considerably towards the trailing part. These changes indicate that the traditional soliton condition fails in the single-cycle regime because of a strong influence of self-steepening. The FDTD method can also produce the same result, but it requires much more computational resources.

One may ask whether the GNLS equation can be used for a single-cycle pulse. As discussed in Ref. [91], this equation can be used for pulses containing a few optical cycles. Something surprisingly, numerical calculations based on GNLS show that it can be applied to pulses containing a single cycle. Computational effort required by the time transformation method is comparable to that required for solving GNLS, 
both of which are much faster than the FDTD method. 


\section{Raman Media: Delayed}

\section{Nonlinearity}

In Chapter 6, we show that the time transformation method could be applied to an optical Kerr medium with instantaneous nonlinearity. As discussed in the introductory chapter, some materials also exhibit a delayed nonlinearity besides the instantaneous nonlinearity. Such a delayed nonlinearity can have a large impact on optical pulse propagation, especially for ultrashort pulses whose width is comparable to the response time of the nonlinear medium.

In this chapter, the time transformation method is extended to include the delayed Raman response and is used to study the propagation of ultrashort, few-cycle, optical pulses. It is first applied to a pulse containing more than 10 optical cycles to show that this new method reproduces the results obtained by solving the NLS equation numerically. The pulse width parameter $T_{0}$ is then varied from 20 to 1 fs to study how the features such as soliton fission, intrapulse Raman scattering, and dispersive-wave generation depend on the pulse width and the soliton order. It is found that for a 
pulse with fixed soliton order, the Raman-induced frequency shift becomes smaller as pulse width decreases, while the fraction of energy transferred to the dispersive wave increases as pulse width is reduced. For the special case of a single-cycle pulse, the most dominant effect is self-steepening and it leads to dramatically new features in both pulse shape and spectrum.

\subsection{Time-transformation by a Raman medium}

As discussed in Chapter 3 that under some reasonable assumptions, the propagation of an optical pulse through a dielectric medium can be described by the linear-system integral relation

$$
E_{\text {out }}(t)=\int_{-\infty}^{\infty} h\left(t, t^{\prime}\right) E_{\text {in }}\left(t^{\prime}\right) \mathrm{d} t^{\prime}
$$

where $E_{\text {in }}$ and $E_{\text {out }}$ are the input and output electric fields associated with the pulse and $h\left(t, t^{\prime}\right)$ is the impulse response function of the dielectric medium. We showed in Chap. 6 that this relation can be applied to a nonlinear Kerr medium using a nonlinear transformation in the time domain. In this approach, the delay of each temporal slice of the electric field inside the nonlinear medium is included through a temporal mapping that is refered to as time transformation. The central issue in this chapter is whether the time transformation method can be used when the medium's nonlinear response is not instantaneous and is delayed by a duration that may exceed the pulse width itself. It turns out that the time transformation method remains valid 
even when the delayed nonlinear response is considered because the simple picture of mapping each input slice to a corresponding output slice continues to hold. The only difference is that the refractive index now depends on light intensity in a delayed fashion.

To be more specific, the following functional form for the medium's nonlinear response is assumed [69]:

$$
R(t)=\left(1-f_{R}\right) \delta(t)+f_{R} h_{R}(t)
$$

where the first term accounts for the instantaneous electronic response (Kerr type) and $h_{R}(t)$ represents the delayed Raman response function of molecules with a fractional contribution $f_{R}$. The refractive index of the medium can be related to $R(t)$ as [92]

$$
n(t)=n_{0}+n_{2} \int_{-\infty}^{t} R\left(t-t^{\prime}\right) I\left(t^{\prime}\right) \mathrm{d} t^{\prime}
$$

where $n_{0}$ is the linear part of the refractive index, $n_{2}$ is the nonlinear coefficient, and $I(t)$ is the intensity. Substituting Eq. (7.3) into Eq. (7.2), one obtains

$$
n(t)=n_{0}+n_{2}\left[\left(1-f_{R}\right) I(t)+f_{R} \int_{-\infty}^{t} h_{R}\left(t-t^{\prime}\right) I\left(t^{\prime}\right) \mathrm{d} t^{\prime}\right]
$$

In the time transformation method, the transit time of a temporal slice located at $t^{\prime}$ is given by $T_{d}=n\left(t^{\prime}\right) L / c$, where $L$ is the medium length and $c$ is the speed of light in vacuum. It follows from the form of Eq. (7.4) that the transit time consists of three parts and can be written as

$$
T_{d}=n\left(t^{\prime}\right) L / c=T_{\text {Linear }}+T_{\text {Kerr }}+T_{\text {Raman }}
$$


The first part of the transit time, $T_{\text {Linear }}=n_{0} L / c$ comes from the linear part of the refractive index and it provides the dominant contribution. The nonlinear part of transit time has two contributions related to the instantaneous and delayed part of the nonlinearity. These are given by

$$
T_{\text {Kerr }}=n_{2} \frac{L}{c}\left(1-f_{R}\right) I\left(t^{\prime}\right)
$$

and

$$
T_{\text {Raman }}=n_{2} f_{R} \frac{L}{c} \int_{-\infty}^{t^{\prime}} h_{R}\left(t^{\prime}-t^{\prime \prime}\right) I\left(t^{\prime \prime}\right) \mathrm{d} t^{\prime \prime}
$$

Using a nonlinear transformation (or mapping) of the time $t^{\prime}$ of a temporal slice time to a new time $t_{1}=t^{\prime}+T_{d}$, one obtains

$$
E_{\text {out }}(t)=\int_{-\infty}^{\infty} h\left(t-t_{1}\right) E^{\prime}\left(t_{1}\right) J\left(t_{1}\right) \mathrm{d} t_{1}
$$

where $E^{\prime}\left(t_{1}\right)=E_{\text {in }}\left(t^{\prime}\right)$ and the Jacobian of this transformation is given by

$$
J\left(t_{1}\right)=\mathrm{d} t^{\prime} / \mathrm{d} t_{1}=\left(1+\mathrm{d} T_{\text {Kerr }} / \mathrm{d} t^{\prime}+\mathrm{d} T_{\text {Raman }} / \mathrm{d} t^{\prime}\right)^{-1}
$$

The important point to note is that the time transformation allows one to write Eq. (7.1) in the form of a convolution. As a results, the integration can be performed numerically in the frequency domain using the convolution theorem, provided the functional form of the impulse response function $h(t)$ is known. As discussed in Section 3.4, the Fourier transform of $h(t)$ is given by $H(\omega)=\exp [i \beta(\omega) L]$, where $\beta(\omega)$ is the propagation constant, defined as $\beta(\omega)=n_{0}(\omega) \omega / c$. Thus, the dispersion nature of the medium is contained fully in its impulse response function $h(t)$. 
In the following numerical calculations, the dispersive effects are considered by expanding the propagation constant $\beta(\omega)$ in a Taylor series around the carrier frequency $\omega_{0}$ of the pulse and retaining terms up to third order [Eq. (2.19)]. For few-cycle pulses, it may be necessary to include other higher-order dispersion terms or even to use the Lorentz model to account for dispersion fully. In this section, dispersion is included only up to third order to emphasise the role of delayed Raman response. The inclusion of higher-order terms does not affect the conclusions here.

\subsection{Comparison with the NLS equation approach}

Before considering single or few-cycle pulses, the time transformation method is first applied to a relatively wide optical pulse containing more than 10 cycles and the results are compared with those obtained using the generalized NLS equation. More specifically, the propagation of a third-order soliton is considered and the input electric field is given by

$$
E_{\text {in }}(t)=\operatorname{Re}\left[\sqrt{I_{0}} \operatorname{sech}\left(t / T_{0}\right) e^{-i \omega_{0} t}\right]
$$

where the carrier frequency is chosen as $\omega_{0} / 2 \pi=200 \mathrm{THz}$, corresponding to an input wavelength of $1.5 \mu \mathrm{m}$. The pulse width parameter is set as $T_{0}=30 \mathrm{fs}$ to ensure that the input pulse has more than 10 cycles and the pulse is assumed to propagate in the anomalous-dispersion region $\left(\beta_{2}<0\right)$ of a silica waveguide, where an optical soliton can form. The peak intensity $I_{0}$ of the pulse is chosen so that it corresponds to a 
third-order soliton [59]: $N=\left(\beta_{0} n_{2} I_{0} L_{D}\right)^{1 / 2}=3$, where $L_{D}=T_{0}^{2} /\left|\beta_{2}\right|$ is the dispersion length. Third-order dispersion is also included using $\delta_{3}=\beta_{3} /\left(6 T_{0}\left|\beta_{2}\right|\right)=0.06$, a typical value for silica waveguides. The Raman effect is included using the functional form of the Raman response function given by Eq. (2.25) [69].
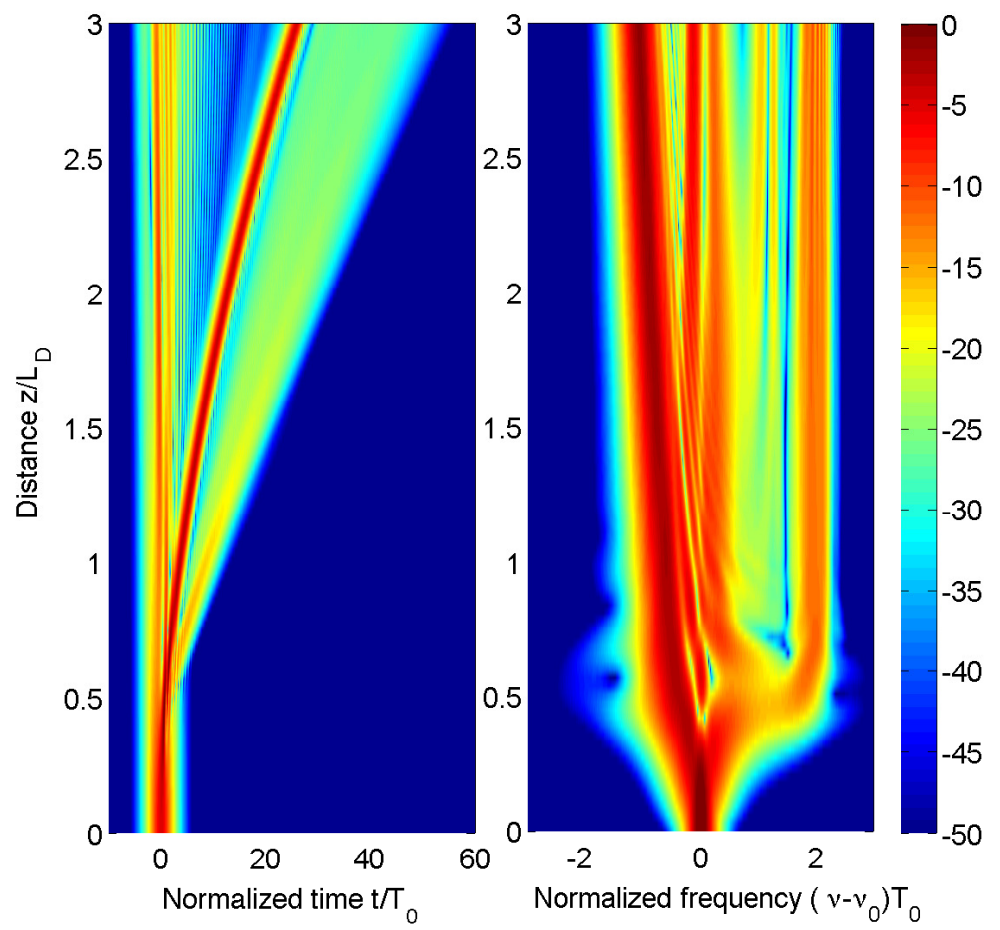

Figure 7.1: Evolution of temporal and spectral profiles over three dispersion lengths when an input pulse with $T_{0}=30 \mathrm{fs}$ forms a third-order soliton. The intensities are plotted using a normalized 50-dB color scale.

Figure 7.1 shows the evolutions of temporal and spectral intensities over three dispersion lengths using time transformation method. Same calculation was repeated by solving the generalized NLS equation and the results were identical in all respects. The fission of third-order soliton occurs near $z=0.5 L_{D}$ in both cases, and the original 
soliton splits into three parts. Most of the energy goes into a much narrower Raman soliton that travels slower than the original pulse as its spectrum shifts continuously toward the red side (the broad peak on the left side). Some energy is also shed in the form of a dispersive wave at a frequency on the blue side of the input pulse spectrum (vertical line on the right side). The location of the blue-shifted peak agrees with the phase-matching condition associated with the generation of a dispersive wave [59]. The important point to stress is that this new method reproduces all known features and agrees completely with the NLS equation results.

To have a better understanding and have a closer look, the pulse shape and spectrum at the output end $z=3 L_{D}$ are plotted in Fig. $7.2(\mathrm{a})$ and (b), respectively. In order to resolve all features, both the pulse shape and spectrum are plotted on a log scale. As shown in part (b), multiple spectral peaks (marked from 1 to 6) are developed after the third-order soliton has propagated for three dispersion lengths. The strongest peak on the red side of the input frequency (peak 1) corresponds to the Raman-shifted soliton peak. Due to the Kerr effect, the original spectral peak now develops into two peaks (2 and 3) on both sides, a typical SPM spectrum. The reason that the energy is larger in the red-shifted peak (2) is that the self-steepening effect and intrapulse Raman scattering break down the symmetry of the SPM process. The spectral peak on the rightmost (peak 6) is the dispersive wave peak and its spectral location agrees with phase-matching condition. There are two new spectral peaks (4 and 5) showing up in the region between the dispersive wave and input spectral peak. 
The presence of these two spectral peaks is the signature of the four wave mixing (FWM) among dispersive wave, the Raman-induced soliton, and light at the original frequency. In fact, one can easily tell from the relative locations and the structures of the spectral peaks that two FWM processes happen that satisfy the following phase matching conditions:

$$
\begin{aligned}
& \beta_{1}+\beta_{6}=\beta_{2}+\beta_{5}, \\
& \beta_{1}+\beta_{6}=\beta_{3}+\beta_{4},
\end{aligned}
$$

where $\beta_{i}(i=1,2, \ldots)$ represents the wave vector for the wave from the $i_{t h}$ spectral peak.
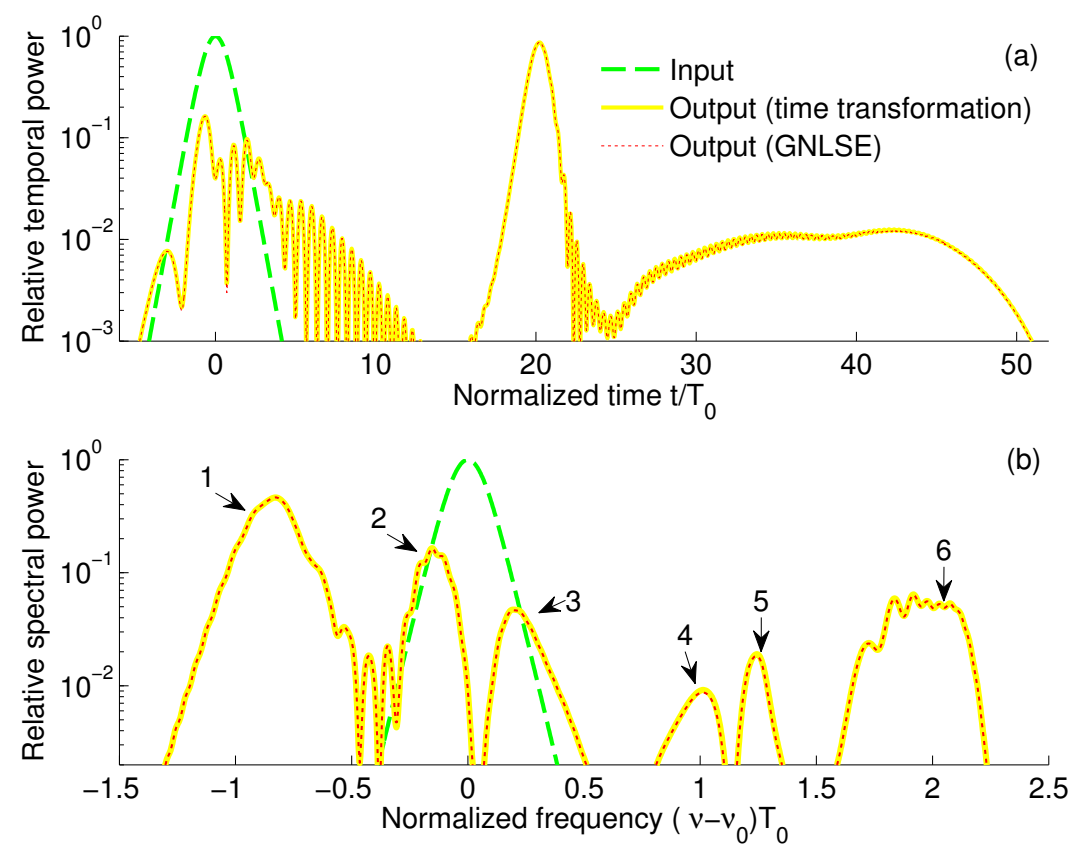

Figure 7.2: Pulse shape and spectrum of the 30 fs third-order soliton in Fig. 7.1 at $z=3 L_{D}$. Predictions of the time transformation method (solid-yellow) agree quite well with those from generalized NLS equation (dotted-red). 
In the temporal domain, much more structure than what is apparent in Fig. 7.1 can now be clearly seen. Besides the three major parts: the Raman soliton (near $\left.t / T_{0}=20\right)$, the dispersive wave (near $\left.t / T_{0}=40\right)$ and the original soliton (near $t=0$ ), one also see some oscillations on the intensity profile within two time windows $-5 T_{0}<$ $t<13 T_{0}$ and $22 T_{0}<t<30 T_{0}$. These oscillations are due to the beating of light at different frequencies. The same calculation was also performed by solving the GNLS equation and the results are plotted by red-dotted curves in Fig. 7.2. As shown in the figure, calculated results overlap perfectly with those from the time transformation method, which indicates that this new method provides an accurate description and is capable of predicting all features for nonlinear dispersive propagation of optical pulses.

\subsection{Propagation of few-cycle pulses}

As seen in the previous section, propagation of short optical pulses inside a nonlinear dispersive medium exhibits rich dynamics through phenomena such as soliton fission, generation of dispersive wave and four-wave mixing [93,94]. The question is what would happen if the pulse duration is reduced till the input pulse contains only a single optical cycle. To answer this question, a series of simulations is carried out by reducing $T_{0}$ in Eq. (7.10) from 20 fs (5 optical cycles) down to 1 fs (single cycle). The relative strengths of nonlinearity and dispersion remain unchanged in the calculation so that the a third-order soliton is formed in all cases. Figure 7.3 shows how the 
temporal shapes (top) and spectra (bottom) at a distance of $z=3 L_{D}$ change as pulses become shorter. As seen there, multiple changes occur, including a decrease in the Raman-induced frequency shift and an increase in energy of the dispersive wave, when $T_{0}$ is reduced from 20 fs to 2 fs. When $T_{0}$ is further decreased down to $1 \mathrm{fs}$, the output pulse exhibits even more drastic features. All such changes are discussed in what follows.

\subsubsection{Intrapulse Raman Scattering}

As seen in Fig. 7.3, for all pulse widths, a large fraction of input energy appears in a Raman soliton that travels slower than the input pulse because its spectrum has been shifted considerably toward the red side of the original spectrum. This change can be expected since the Raman response time of about 60 fs for silica fibers is considerably larger than the pulse width. As the pulse becomes narrower, the influence of the delayed Raman response gets smaller. As a consequence, the Ramaninduced frequency shift decreases for narrower pulses, which in return decreases the difference between the group velocities of the Raman soliton and rest of the pulse. The spectral shift of the Raman soliton decreases almost linearly until $T_{0}=10 \mathrm{fs}$. When $T_{0}$ becomes shorter than this value, one cannot even see a clear spectral peak for the Raman soliton because it does not separate enough from the input spectrum.

To obtain the correct magnitude the Raman-induced frequency shift as a function of pulse width $T_{0}$, the Raman soliton is targeted in the temporal domain, where a 

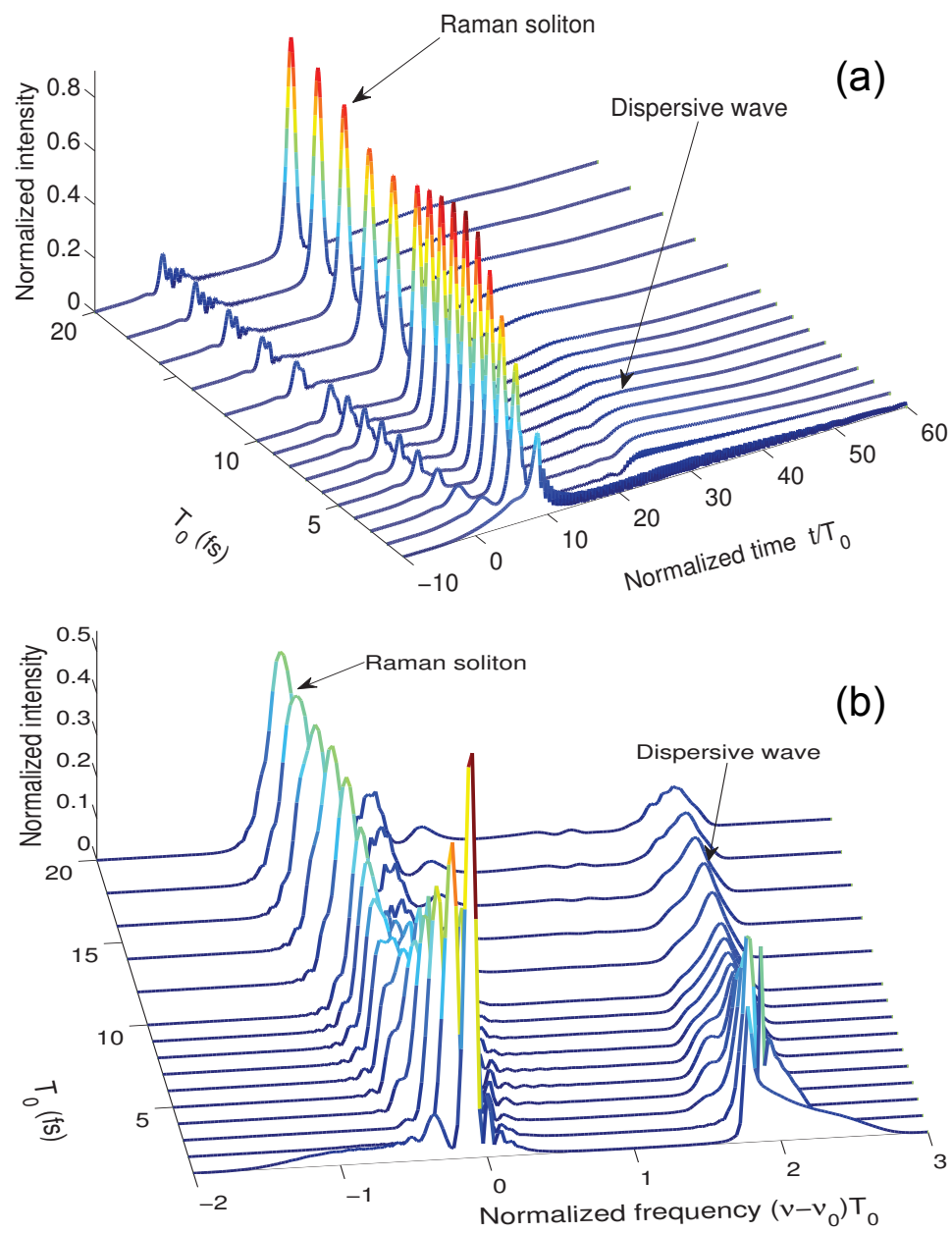

Figure 7.3: Comparison of pulse shapes (a) and spectra (b) at $z=3 L_{D}$ as $T_{0}$ is reduced from 20 to 1 fs. The input pulse corresponds to a third-order soliton in all cases.

clear intensity peak is seen even down to $T_{0}=1 \mathrm{fs}$. By taking the Fourier transform of only this part of the pulse, the spectral shift of the Raman soliton is deduced. Figure 7.4 shows the Raman-induced frequency shift as a function of $T_{0}$ for soliton orders of $N=2,3$, and 4 . In these calculations, the peak intensity of the input pulse is increased to correspond to different soliton order, while other parameters $\left(n_{2}, \beta_{2}\right.$, and 


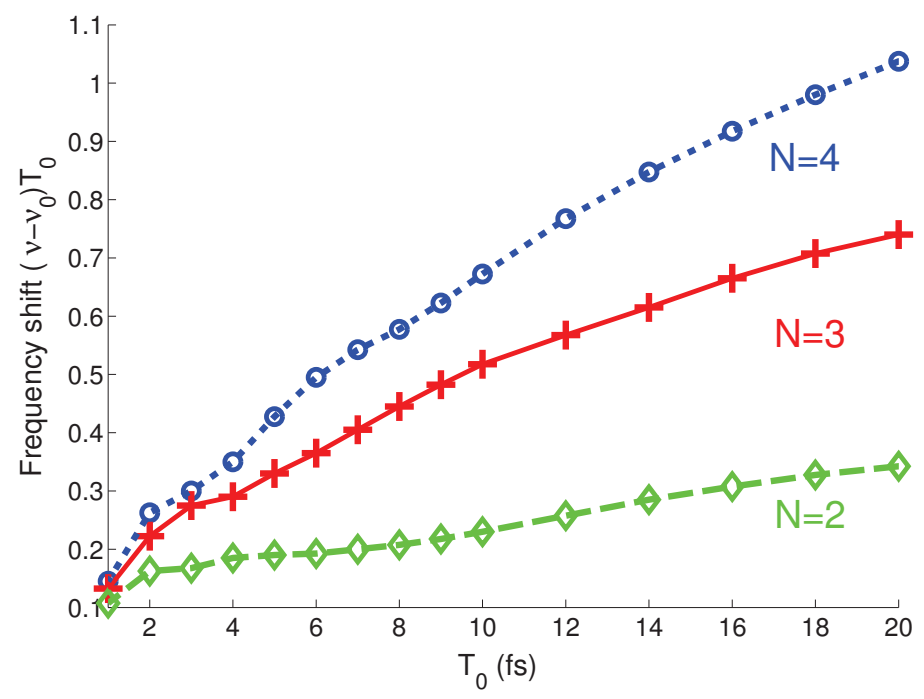

Figure 7.4: Changes in the Raman-induced frequency shift as $T_{0}$ is reduced from

20 to 1 fs for a fixed soliton order $N$. For the same value of $T_{0}$, pulses with a higher soliton order have larger frequency shifts.

$\left.\beta_{3}\right)$ are kept unchanged. It is expected that pulses with a larger $N$ value would have larger Raman-induced frequency shifts because of their higher peak powers. As seen in Fig. 7.4, the amount of Raman-induced frequency shift decreases monotonically with decreasing $T_{0}$ for all three values of $N$. This is opposite to what is observed for pulses wider than the Raman response time of $60 \mathrm{fs}$, for which the Raman-induced frequency shift is known to scale as $T_{0}^{-4}[70]$. This qualitative difference between the short and long pulses is a manifestation of the fact that intrapulse Raman scattering is not an instantaneous nonlinear process and its response is delayed by about $60 \mathrm{fs}$ in the case of silica glass. 


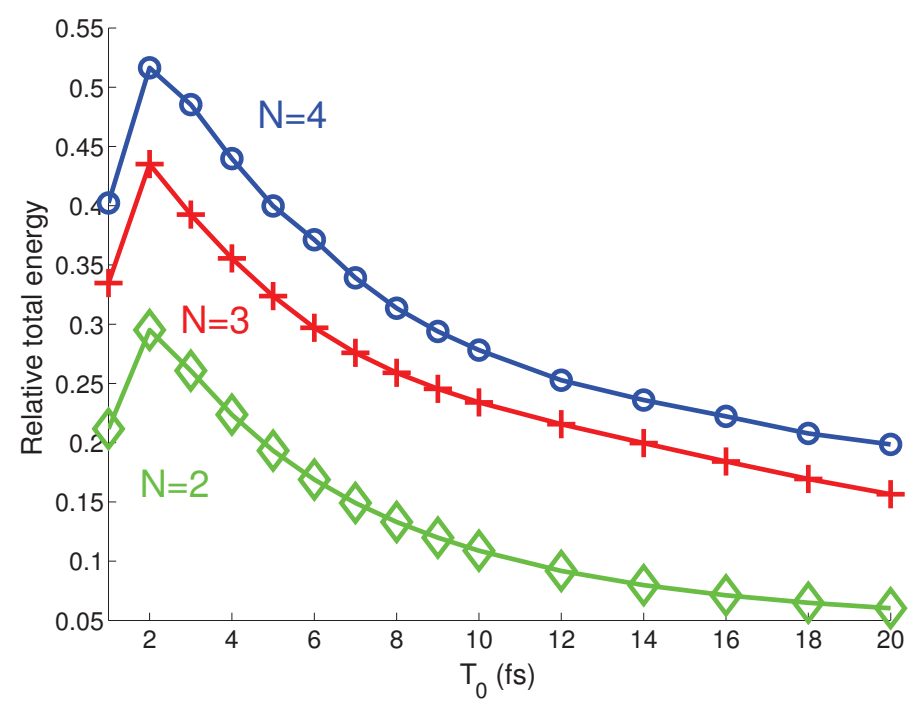

Figure 7.5: Energy of the dispersive wave as a function of $T_{0}$ for $N=2,3$, and 4. The drop for $T_{0}<2$ fs is related to the development of rapid temporal oscillations.

\subsubsection{Dispersive Wave Generation}

A significant feature of Fig. 7.3 is that, as $T_{0}$ is reduced from 20 fs to 2 fs, more and more of the input pulse energy is transferred to a dispersive wave at a blue-shifted wavelength. This feature was also observed in Ref. [94] that focused on pulse longer than 10 fs. Here it is found that it persists for pulses as short as a single optical cycle. This feature can be clearly seen in the spectral domain in Fig. 7.3(b). To find the energy of this spectral peak, the spectral power density is integrated over the frequency range $1<\left(\nu-\nu_{0}\right) T_{0}<3$ for different orders of solitons $(N=2,3$, and 4$)$ with $T_{0}$ in the range of $1-20$ fs. The results are shown in Fig. 7.5.

As seen in Fig. 7.5, a higher-order soliton transfers more of its input energy into a 
dispersive wave. This feature agrees with the previous findings and is expected if one recalls that a dispersive wave is generated by the perturbation of an optical soliton by the third-order dispersive effect. However, more interesting is the observation that, for a given value of $N$, more energy can be transferred to a dispersive wave by reducing the input pulse width. One can see in Fig. 7.5 that more and more energy is transferred to the dispersive wave as $T_{0}$ decreases. However, the situation changes when $T_{0}$ is reduced to below 2 fs. Further investigation shows that this change is related to the impact of self-steepening. As the pulse width decreases, although the Raman response becomes weaker, the impact of self-steepening effect governed by the parameter $\left(\omega_{0} T_{0}\right)^{-1}$ becomes stronger. For example, the value of the self-steepening factor is only 0.04 for 20 fs pulses, but it approaches 0.8 for $T_{0}=1$ fs at a carrier frequency of $200 \mathrm{THz}$. It turns out that that when the self-steepening factor exceeds 0.5, self-steepening leads to drastic changes in both in the shape and the spectrum of optical pulses. Such changes are apparent in Fig. 7.3 when one compares the curves for $T_{0}=1$ fs and 2 fs. Since a pulse with $T_{0}=1$ fs contains only a single optical cycle, the next section focuses on single-cycle pulses.

\subsection{Propagation of single-cycle pulses}

As seen in Fig. 7.3, the temporal and spectral features change drastically when $T_{0}$ is reduced from 2 fs to 1 fs. In the temporal domain, the distinction between the original pulse, the Raman soliton and the dispersive wave disappears, and one can only see 
a single dominant peak with a long tail exhibiting rapid oscillations. The pulse spectrum becomes very asymmetric and develops many new frequency components both on the red side of the original carrier frequency peak and on the blue side of the dispersive-wave peak. This section focuses these new features occurring when the input pulse contains a single optical cycle.

For single-cycle pulses, the concept of pulse envelop begins to loose its meaning. For this reason, the actual electric field associated with the pulse is plotted. This is straightforward in the time transformation method since it deals directly with the electric field. Figure 7.6(a) shows the output electric field (solid red curve) at $z=3 L_{D}$ for a single-cycle input pulse, together with the input electric field (dotted green curve). As seen there, the electric field for $t<5 T_{0}$ appears to be a stretched version of the input electric field with reduced amplitude. However, for $t>5 T_{0}$, the behavior of electric field becomes quite complicated. This is also reflected in part (b) where temporal intensity is plotted on a logarithmic scale, and high-frequency oscillations on a relatively long pulse tail are evident starting around $t=8 T_{0}$. These oscillations result from a beating between the frequency components near the input carrier frequency and those associated with the blue-shifted dispersive wave. They also occur for multi-cycle pulses but their amplitude is negligibly small.

To understand this phenomenon more clearly, Fig. 7.7 shows a spectrogram of the pulse exhibiting temporal and spectral features simultaneously. A Gaussianshape sampling window function $W(t, \tau)$ is adopted to obtain spectral information 

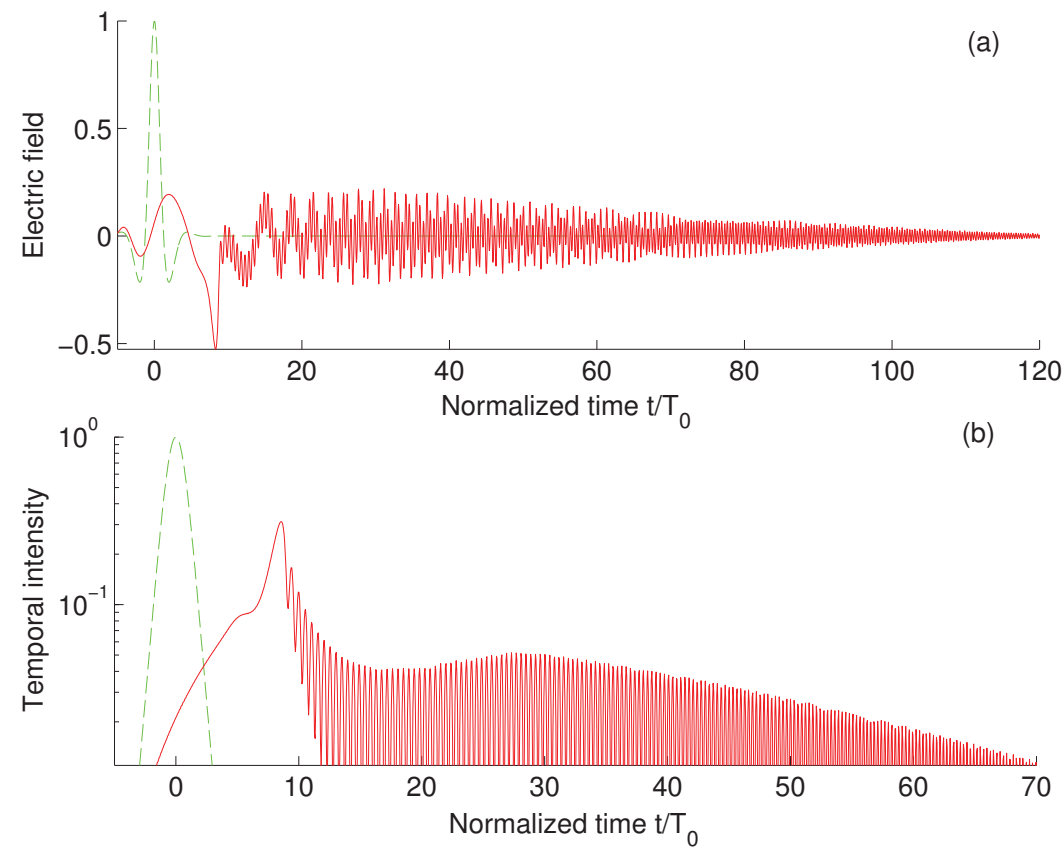

Figure 7.6: (a) Electric field and (b) temporal intensity at a distance of $3 L_{D}$ when a third-order soliton is excited using input pulse with $T_{0}=1 \mathrm{fs}$. The input electric field and intensities are shown for comparison by dotted green curves.

for different temporal slices and the spectrogram is calculated using

$$
S(\omega, \tau)=\left|\int_{-\infty}^{\infty} W(t, \tau) E(t) e^{i \omega t} \mathrm{~d} t\right|^{2}
$$

Two spectral peaks are clearly seen, corresponding to spectral components around the carrier frequency and the frequency of the generated dispersive wave, respectively. Since these two peaks overlap in time, they interfere and produce temporal fringes in the time domain. A wing-like structure is generated because the two spectral peaks fall on opposite sides of the zero-dispersion wavelength. This spectrogram explains the behavior of the electric field in Fig. 7.6. For example, for $t<5 T_{0}$, only one spectral 
peak occurs that is slightly red-shifted because of intrapulse Raman scattering. After this point, multiple spectral components co-exist together, and it is the beating among them that leads to distortion of the electric field and rapid oscillations in the pulse tail. Indeed, the oscillation frequency in the pulse tail in Fig. 7.6(b) agrees well with the frequency difference between the blue-shifted dispersive wave and red-shifted Raman soliton seen in Fig. 7.7.

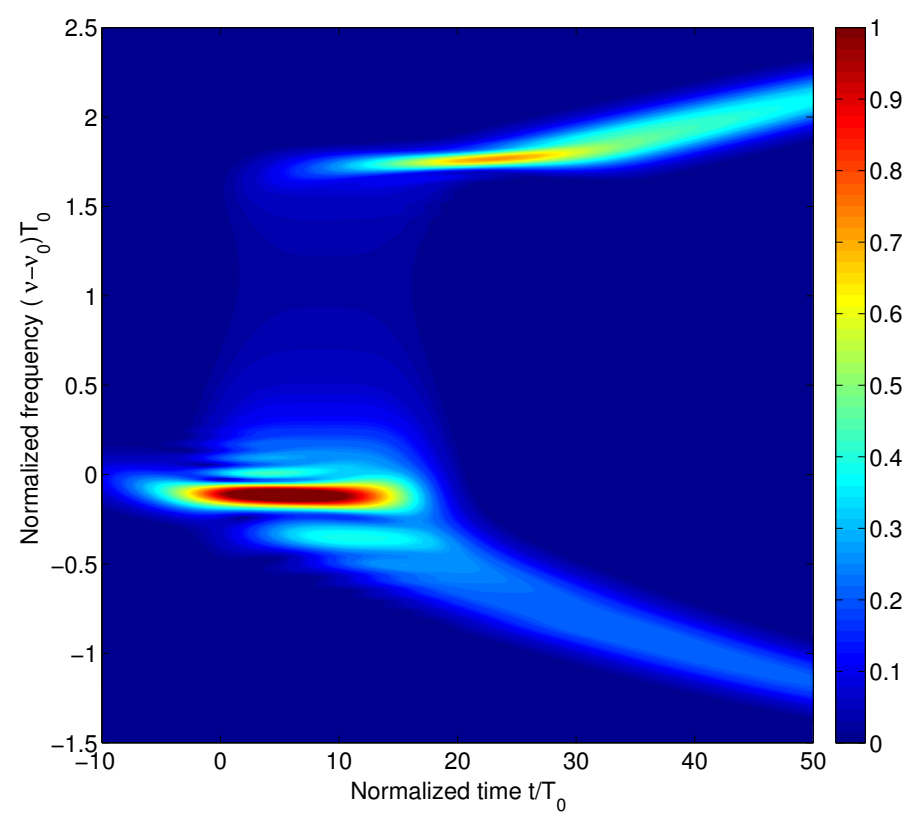

Figure 7.7: Spectrogram at $z=3 L_{D}$ for the $T_{0}=1$-fs case shown in Fig. 7.6.

The intensity is plotted on a linear color scale.

The spectrogram helps us in understanding the origin of rapid oscillations in the pulse temporal profile. However, one question still remains: what mechanism is responsible for the generation for such drastic changes in the electric field of singlecycle pulses? The answer turns out to be the self-steepening effect. As the pulse width 
is reduced, the relative strength of dispersion scales accordingly since the normalized versions of $\beta_{2}$ and $\beta_{3}$ are employed in the numerical study. The nonlinearity strength is also fixed because the soliton order $N$ does not change. The only effect that is magnified for single-cycle pulses is the self-steepening effect. Indeed, the selfsteepening factor takes a relatively large value of 0.8 for single-cycle pulses. To confirm that indeed self-steepening is behind the drastic changes, the simulation for single-cycle pulses is repeated by neglecting the self-steepening effect, while keeping other nonlinear effects. The resulting pulse shape and spectrum are plotted by the dashed red curves in Fig. 7.8. For direct comparison, the solid blue curves show results when everything is included and dotted green curves show the input pulse.

As seen in Fig. 7.8, when self-steepening is absent, three separate regions in the temporal domain are clearly seen that correspond to the residual input pulse near $t=0$, a Raman soliton near $t=10 T_{0}$, and a dispersive wave near $t=30 T_{0}$. In the spectral domain, one can also clearly see the corresponding spectral components. Specially, the red-shifted Raman soliton peak and a blue-shifted peak that corresponds to the dispersive wave can clearly be seen. However, when self-steepening is present, both the temporal and spectral intensity profiles change dramatically. In particular, in the time domain the pulse exhibits a single dominant peak, together with an oscillatory tail that extends over a range exceeding $70 T_{0}$. The peak amplitude is reduced considerably because a large fraction of of the input pulse energy is now contained in the long oscillatory tail. This is also evident in the spectral domain, 

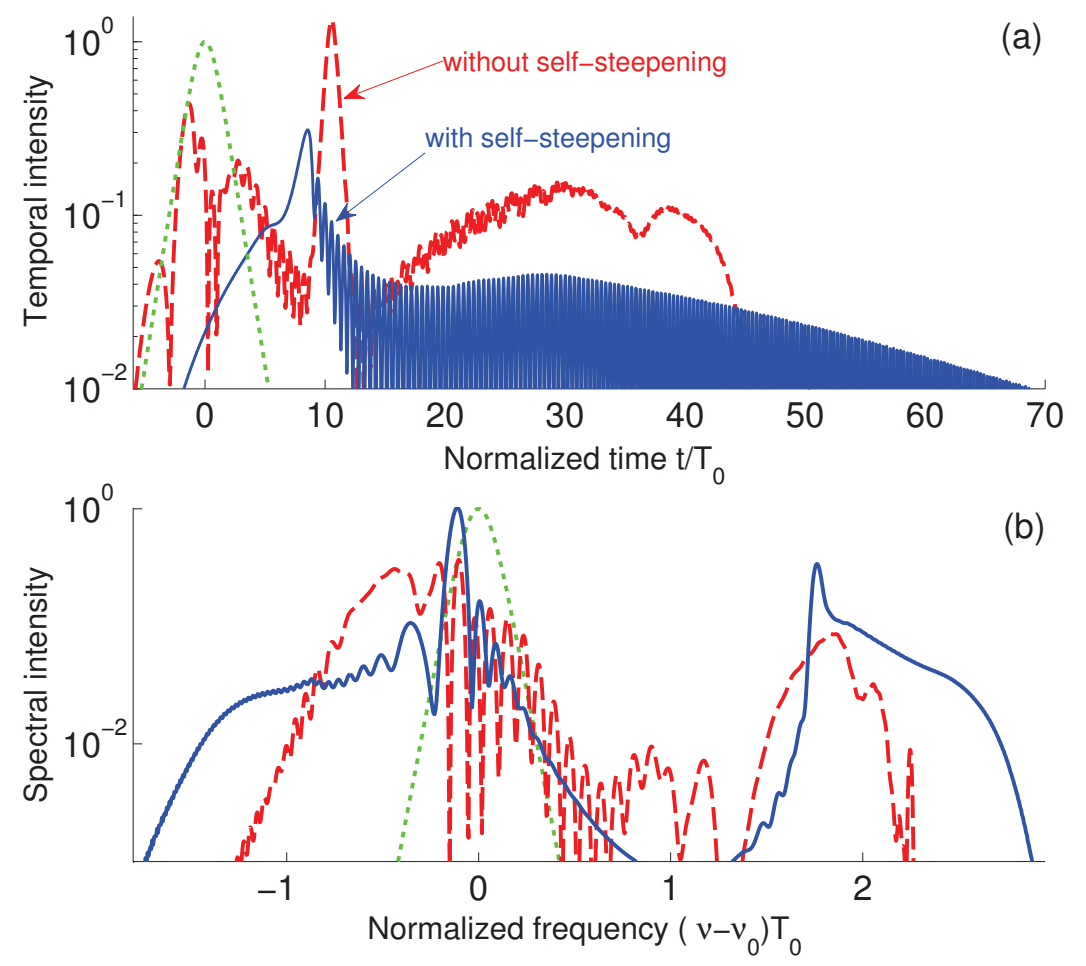

Figure 7.8: Impact of self-steepening on the shape (a) and spectrum (b) of a single-cycle input pulse. Self-steepening is turned off artificially for the dashed red curve but is included fully for the solid blue curve. The input curves are shown by dotted-green curves.

where the blue-shifted peak that corresponds to the dispersive wave is much broader with an asymmetric tail. Therefore, we come to the conclusion that for single-cycle pulses self-steepening becomes so dominant that it overtakes other nonlinear effects and modifies the evolution of pulse shape and spectrum in a drastic fashion. 


\section{Kerr Nonlinearity for}

\section{Few-Cycle Pulses}

In this chapter, the time transformation method is applied for studying the propagation of few-cycle optical pulses inside a nonlinear Kerr medium after taking into account that changes in the refractive index vary with the electric field as $E^{2}$, and not by its average over an optical cycle $\left\langle E^{2}(t)\right\rangle$. The time transformation method correctly predicts carrier-wave shocking and generation of odd-order harmonics inside a Kerr medium, the two features found earlier by solving Maxwell's equations using the finite difference time domain (FDTD) method. This method is further extended to study the impact of a finite response of the Kerr nonlinearity on harmonic generation and to include chromatic dispersion that cannot be ignored for ultrashort pulses. 


\subsection{Nonlinearity without averaging}

The development of ultrafast optical technology enables the generation of ultrashort pulses containing only a few cycles of the electromagnetic field. Many applications require propagation behavior of such short pulses inside a nonlinear dispersive Kerr medium. Although the nonlinear Schrödinger (NLS) equation, derived from Maxwell's equations using the slowly varying envelope approximation [59], cannot be used for such short pulses, a generalized version of this equation has been used with success for pulses as short as single cycle [91]. However, in this envelope-based approach, the Kerr nonlinearity is included using the form

$$
n=n_{0}+n_{2} I(t)=n_{0}\left\{1+\frac{n_{2} c \epsilon_{0}}{2}\left\langle E^{2}(t)\right\rangle\right\}
$$

for the refractive index, where $n_{2}$ is the Kerr parameter and the average is over an optical cycle. Although it is possible to extend the envelope approach further by adding additional nonlinear terms [95], it is common to employ the FDTD method and solve Maxwell's equations directly in the time domain [96]. The FDTD approach shows that the use of $E^{2}(t)$ in place of its average leads to carrier wave shocking and generation of odd-order harmonics when a pulse propagates inside a Kerr medium [97]. However, the use of FDTD algorithm requires a step size that is a small fraction of wavelength $\lambda$. As a result, its use becomes time-consuming for distances much longer than $\lambda$. The time transformation method deals directly with the electric field and should be applicable to such cases. It is also faster numerically compared to the 
FDTD technique.

Without the averaging in time, the refractive index for a nonlinear medium becomes

$$
n(t)=n_{0}+n_{2}^{\prime} \int_{-\infty}^{t} R\left(t-t^{\prime}\right) E^{2}\left(t^{\prime}\right) \mathrm{d} t^{\prime},
$$

where $R(t)$ is the third-order nonlinear response function of the medium [92], and $n_{2}^{\prime}=n_{2} n_{0} c \epsilon_{0} / 2$. The time delay then has two parts, $T_{d}=T_{l}+T_{n l}$. The linear part, $T_{l}=n_{0} L / c$, comes from the linear part of the refractive index and the nonlinear part for a nonlinear medium is given by

$$
T_{n l}(t)=\frac{n_{2}^{\prime} L}{c} \int_{-\infty}^{t} R\left(t-t^{\prime}\right) E^{2}\left(t^{\prime}\right) \mathrm{d} t^{\prime},
$$

Because of the use of $E^{2}$, instead of its cycle-average value, the nonlinear transit time $T_{n l}$ oscillates rapidly at a frequency twice that of the input field. As will be seen later, these rapid oscillations generate odd-order harmonics within a nonlinear medium.

\subsection{Carrier wave shocking and harmonic genera- tion}

As a simple test of the validity of the time transformation method, we neglect the Raman contribution and consider first a non-dispersive Kerr medium. The Kerr medium is assumed to respond instantaneously and $R\left(t-t^{\prime}\right)=\delta\left(t-t^{\prime}\right)$ in Eq. (8.3). 

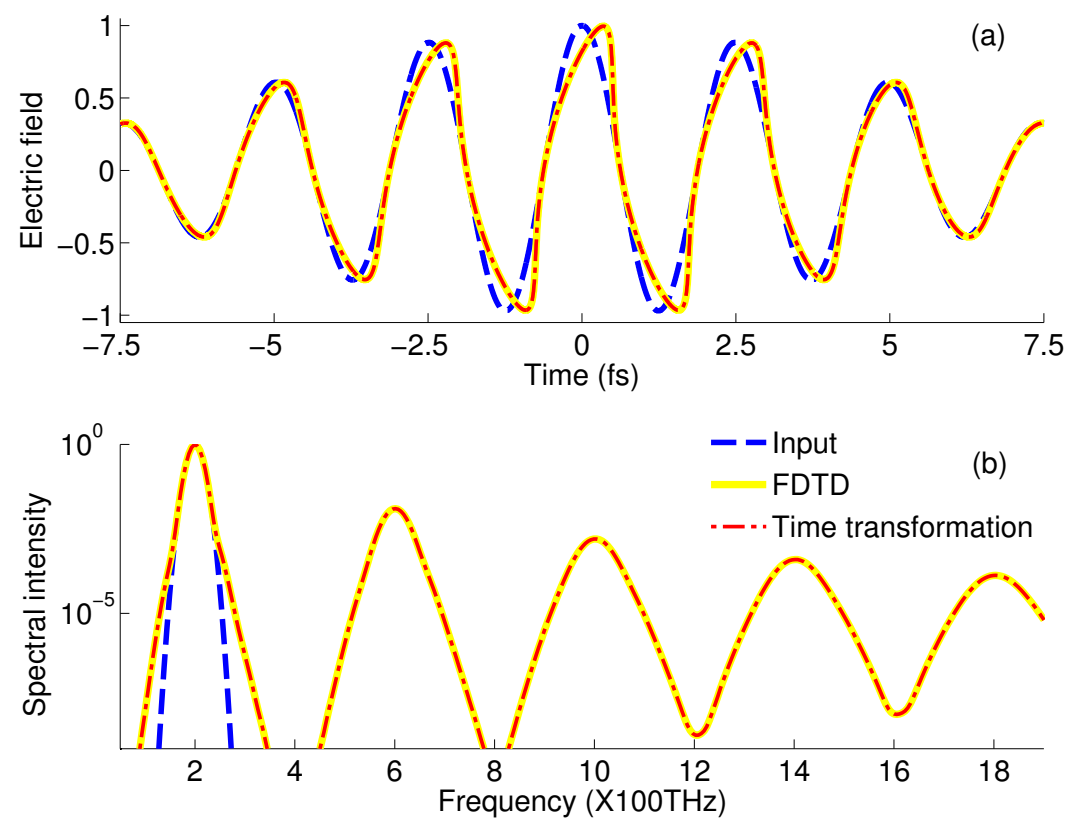

Figure 8.1: (a) The electric fields and (b) optical spectra at the input (dashed blue curves) and output ends of a nondispersive Kerr medium using time transformation approach (dot-dashed red) and the FDTD method (solid yellow).

The input field oscillating at the frequency $f_{c}=200 \mathrm{THz}$ has a Gaussian envelope with a width parameter $T_{0}=5$ fs:

$$
E_{\text {in }}(t)=E_{0} \exp \left[-t^{2} /\left(2 T_{0}^{2}\right)\right] \cos \left(2 \pi f_{c} t\right)
$$

The peak amplitude $E_{0}$ of the electric field is set by the maximum nonlinear phase shift it experiences, $\phi_{\max }=2 \pi f_{c} n_{2}^{\prime} E_{0}^{2} L / c=0.3$.

Figure 8.1 shows (a) the electric fields and (b) corresponding optical spectra at the input and output ends of the Kerr medium using the time transformation method and compares them with those obtained with the FDTD method. The agreement between the two is excellent. The output electric field is distorted because of carrier- 
wave shocking [97], which happens mainly near the pulse center, where the Kerr effect is the strongest. The distortion of the electric field is due to the generation of oddorder harmonics that are seen clearly in Fig. 8.1(b) up to $9 f_{c}$. Physically speaking, a propagating optical pulse inside a Kerr medium modulates the refractive index in a periodic fashion at $2 f_{c}$, creating a moving index grating, which creates spectral sidebands seen in Fig. 8.1(b). Mathematically, the change in the refractive index can be expressed as

$$
\Delta n=\frac{1}{2} n_{2}^{\prime} E_{0}^{2} \exp \left(-t^{2} / T_{0}^{2}\right)\left[1+\cos \left(4 \pi f_{c} t\right)\right]
$$

The first term (without $f_{c}$ ) is responsible for self-phase modulation, while the second term generates odd-order harmonics. Although these two terms are of the same order of magnitude, the later one has much larger impact because it modulates in a much shorter time (optical cycle) as compared to the former one (pulse duration).

\subsection{Impact of finite Kerr response time}

The instantaneous response of a Kerr medium is clearly an approximation because any electronic response should be delayed by some finite time. While this approximation is justified for relatively wide pulses, it becomes questionable for ultrashort pulses. To relax it, a finite response time $\tau_{k}$ is introduced for the Kerr nonlinearity through a commonly used Debye model [98]. It assumes the following exponential form of the 
response function $R(t)$ in Eq. (8.2):

$$
R(t)=\tau_{k}^{-1} \exp \left(-t / \tau_{k}\right)
$$

In the limit $\tau_{k}=0, R(t)$ reduces to a $\delta$ function, as expected. To see the impact of the finite Kerr response time, a series of simulations is performed where $\tau_{k}$ is reduced from 2 fs to 0.05 fs, while all other parameters are kept the same as in Fig. 8.1. Figure 8.2 shows how the peak amplitudes of the $3 \mathrm{rd}, 5$ th, and 7 th harmonics vary with $\tau_{k}$. As seen there, the amplitudes of all harmonics decrease almost exponentially as $\tau_{k}$ increases (notice the semi-log nature of the plot), but the rate of decrease is different for different harmonics. The third harmonic decreases the least, and the rate becomes larger for higher-order harmonics. This behavior can be understood by noting that the $\Delta n$ in Eq. (8.5) oscillates at a frequency of $400 \mathrm{THz}$. In order to resolve these dynamics, the response time should be well below 2.5 fs.

\subsection{Inclusion of dispersion: soliton propagation}

This section considers the case of a dispersive Kerr medium since dispersion cannot be ignored for ultrashort pulses. As is well known, optical solitons can form in a dispersive Kerr medium with a delicate balance between the nonlinear and dispersive effects and when pulses with a specific shape and peak intensity are launched. It is not expected that this balance will persist for few-cycle pulses that are effected by higher-order effects such as self-steepening and higher-order dispersion. Nonetheless, 


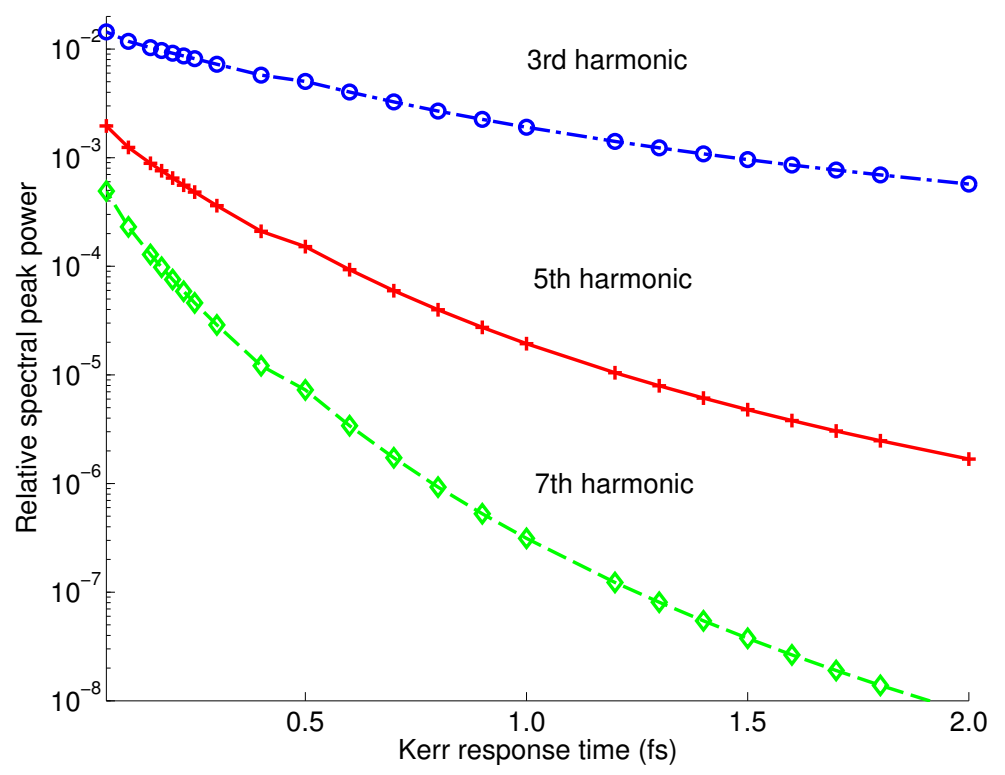

Figure 8.2: Changes in the relative amplitudes of the first three harmonics with the Kerr response time $\tau_{k}$. In all cases, the amplitude decreases almost exponentially with increasing $\tau_{k}$.

it is possible that pulses will remain close to their original shape. To see this effect numerically, an optical pulse with a sech-shape envelope is considered by replacing the Gaussian factor in Eq. (8.4) with $\operatorname{sech}\left(t / T_{0}\right)$ and use $T_{0}=10$ fs. In order to include all-order dispersive effects for such ultrashort pulses, the Lorentz model is employed, for the dielectric constant of the Kerr medium in the form [98]

$$
\epsilon(\omega)=\epsilon_{\infty}+\frac{\omega_{0}^{2}\left(\epsilon_{s}-\epsilon_{\infty}\right)}{\omega_{0}^{2}-i \delta \omega-\omega^{2}}
$$

The four parameters appearing in this model are chosen as: $\epsilon_{s}=5.25, \epsilon_{\infty}=2.25$, $\omega_{0}=6 \times 10^{14} \mathrm{rad} / \mathrm{s}$, and $\delta=2 \times 10^{9} \mathrm{~s}^{-1}$. The second-order dispersion parameter $\beta_{2}$ of such a Kerr medium varies from -4 to $-20 \mathrm{ps}^{2} / \mathrm{m}$ over the frequency range 180-220 
$\mathrm{THz}$, a range that includes $f_{c}=200 \mathrm{THz}$ used in the numerical simulation. The peak amplitude $E_{0}$ in Eq. (8.4) is chosen to satisfy the soliton-formation condition [59] and is found using $n_{2} E_{0}^{2}=0.0182$.
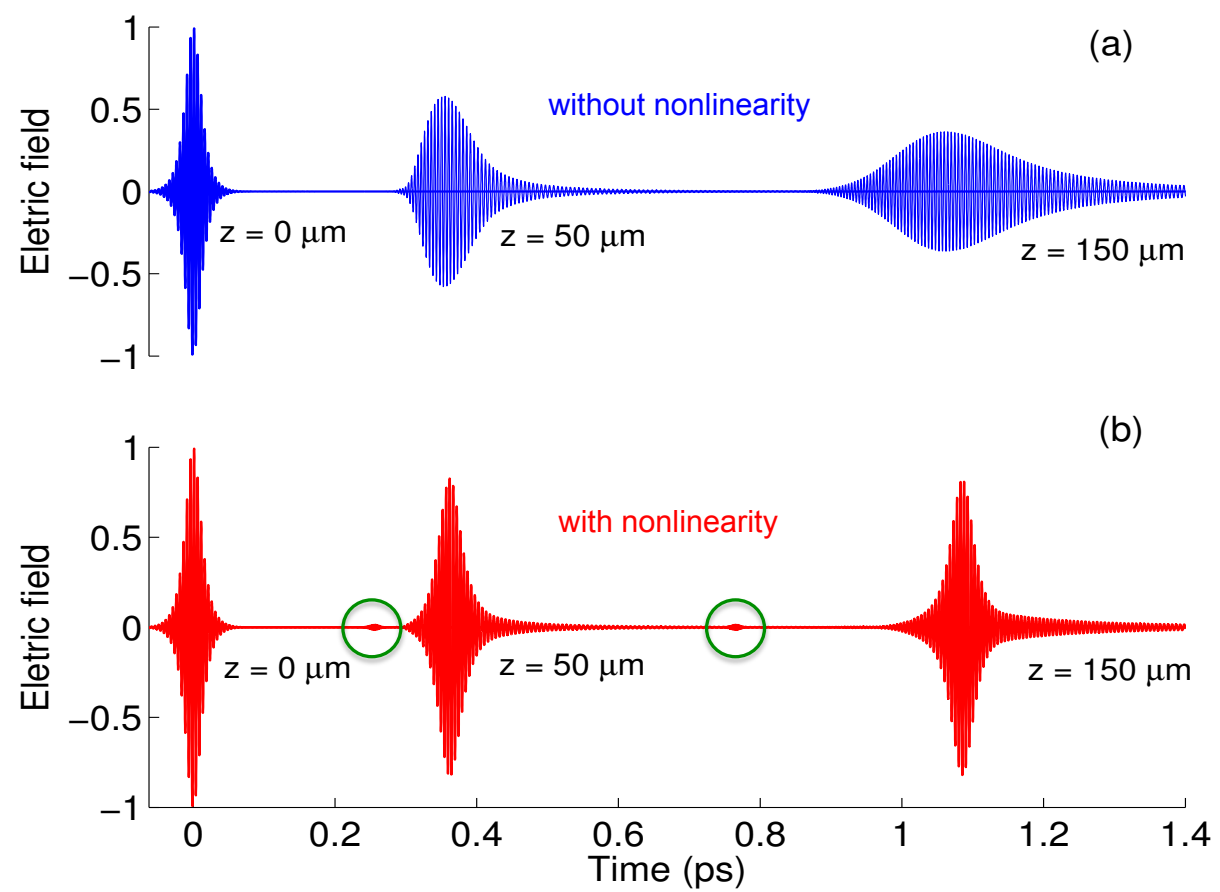

Figure 8.3: Electric fields of a 10 fs optical pulse after it has propagated for $50 \mu \mathrm{m}\left(4 L_{D}\right)$ and $150 \mu \mathrm{m}\left(12 L_{D}\right)$ in a linear (a) and nonlinear (b) dispersive medium. Nonlinearity balances the linear dispersion and helps maintaining the pulse width.

To see whether soliton-like features persist, this few-cycle pulse is propagated in such a dispersive Kerr medium for distance up to $150 \mu \mathrm{m}$ and is compared with the results when nonlinearity is removed by setting $n_{2}=0$. Figure 8.3 shows the electric field of the pulse in these two cases at distances of 50 and $150 \mu \mathrm{m}$, corresponding to 
$4 L_{D}$ and $12 L_{D}$ respectively, where $L_{D}=T_{0}^{2} /\left|\beta_{2}\right|$ is the dispersion length [59]. In a dispersive medium without any nonlinear effects $\left(n_{2}=0\right)$, pulse broadens rapidly as expected (a). However, when the Kerr nonlinearity is included, although increases slightly from 0 to $50 \mu \mathrm{m}$, pulse width remains unchanged from 50 to $150 \mu \mathrm{m}$, indicating soliton-like propagation (b). It is also clear from Fig. 8.3 that the pulse does not maintain its original shape fully and develops a long tail whose origin is related to the presence of higher-order dispersive effects and self-steepening.

A weak "daughter" pulse that oscillates at a much higher frequency can also be seen in Fig. 8.3 (b). The origin of this daughter pulse lies in the odd-order harmonics generated by the Kerr nonlinearity (see Fig. 8.1). In the non-dispersive case, all frequency components overlap temporally, leading to carrier-wave shocking. In a dispersive medium, because of the frequency-dependent group velocity, various harmonics travel at different speeds and form a "daughter" pulse, which separates from the main pulse. This separation becomes larger with further propagation, as seen in Fig. 8.3.

As a further check of the accuracy of the results in Fig. 8.3, the same propagation problem is solved using the FDTD method. Figure 8.4 compares the FDTD results with those obtained by the time transformation method at a distance of $50 \mu \mathrm{m}$. Both the amplitude and phase of the transmitted electric fields agree quite well. However, the time transformation method has a distinct advantage over the FDTD method in terms of the computation speed. For example, the time transformation method took 


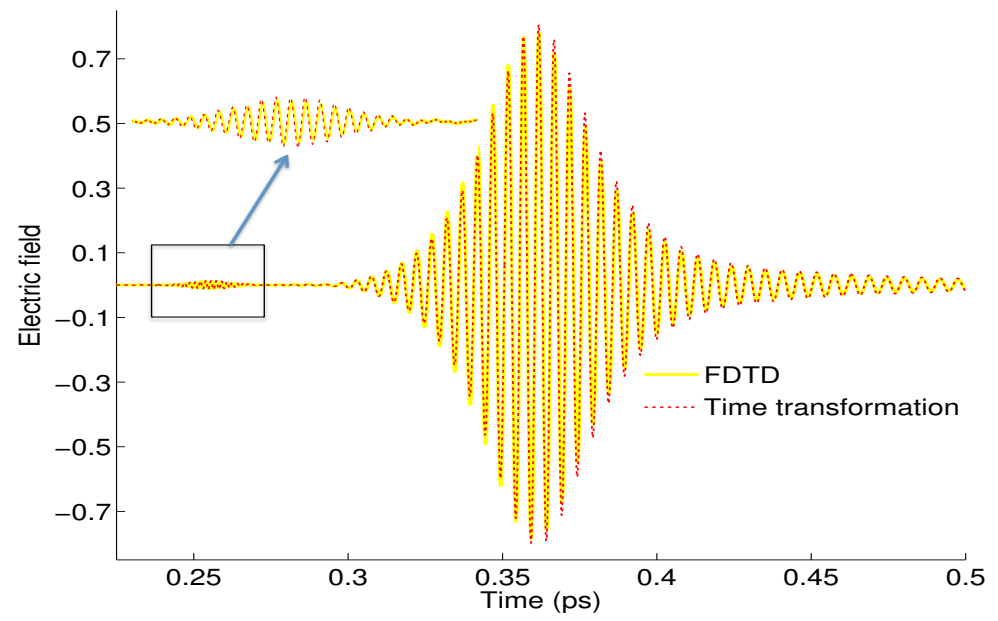

Figure 8.4: Comparison of time transformation (dashed red) and the FDTD (solid yellow) methods. Insert: expanded view of the "daughter" pulse oscillating at high frequencies.

less than 10 seconds in Matlab (version 7.8) while the FDTD method needed more than 100 seconds for attaining the same accuracy for the results shown in the inset of Fig. 8.4. This difference becomes even more pronounced for longer propagation distances. The reason is that the section length in our method is not related to the temporal resolution employed. 


\section{Conclusions and Final}

\section{Thoughts}

This thesis presented a time transformation method to study the pulse propagation problem. In the cases of dynamic linear media, we show that time transformation can be applied both to dynamic traveling-waveguides and resonators to explain the phenomena of adiabatic wavelength conversion happening inside such dynamic linear media. In the case of nonlinear media, time transformation method correctly predicts all nonlinear effects and agrees with the prediction of the traditional NLS equation approach and the FDTD calculation of Maxwell's equations. More importantly, this method maps the electric field directly making it more general than the envelopebased methods; it does not require a step size small compared to light wavelength, making it numerically faster than the FDTD approach. Looking for the future, two final thoughts about this thesis should be brought out into mind.

The first is related to adiabatic wavelength conversion in dynamic linear media. As was discussed in Chap. 2, devices based on such a phenomena can be candidates 
for wavelength converters. Compared to the conventional wavelength converters that use nonlinear effects, these new devices operate at any power level, and do not require the phase matching condition. Most important of all, since the amount of wavelength change is linearly proportional to the change in the refractive index of the medium, such devices can control the amount of wavelength change very conveniently.

However, in reality, such wavelength converters have a relatively small wavelength change range due to the amount by which refractive index can be changed. The refractive index can be changed through the electro-optic effect (injection of free charge carriers in a semiconductor, the Pockels effect, etc.) or a purely optical effect (free carrier generation through optical absorption, cross-phase modulation, etc.), and is typically $<0.01 \%$. In real application, much larger wavelength shifts are needed and this is the major reason that makes wavelength converters based on AWC not suitable for real applications. This restriction might be overcome in the future with advances in material science. AWC may also find applications in plasmas, where changes in the propagation of light speed can be much larger than a dielectric medium. In fact, earlier experimental efforts on AWC were focused on plasmas [14]. Also, one does not really need to change the refractive index to realize AWC. AWC can also happen when the effective propagation speed of light changes. AWC has been realized by shifting the cavity resonance mechanically $[35,36]$ or by shifting the waveguide mode [3]. By only slightly changing the refractive index, the mode structures might be modified considerably, and much larger wavelength shift could be achieved. This research 
direction is far from being fully explored, and lots of work can be done in the future.

The second part of the thesis is devoted to nonlinear media. As discussed in Chaps. 1 and 2, ultrashort optical pulses are routinely used in a variety of areas as the development of laser pulse technology. Relevant models are needed to characterize the propagation of these ultrashort pulses, as well as their interactions with varies media. Direct solutions of Maxwell's equation with the FDTD technique can be used for this purpose, but its use requires lots of computation resources, and also it cannot provide much physical insight. On the other hand, the use of the NLS equation in this region becomes questionable because of SVEA.

The proposed time transformation method in this thesis might be a good candidate for modeling the propagation of such ultrashort pulses because it directly works with the electric field, does not assume SVEA and also it is much faster than FDTD. Looking forward, one can expect new physics to show up owning to the break down of the SVEA. For example, a novel concept of "negative-frequency resonant radiation" was proposed and experimentally confirmed in a recent work [99]. These new effects are unique to ultrashort pulses whose spectrum has a substantial zero-frequency (dc) component. With the investigation into such ultrashort pulses, more interesting physics should be discovered, and one expects that the time transformation method would find more applications in this region. 


\section{A Time Transformation for Pulse}

\section{Envelopes}

This thesis has developed a time transformation method for solving the pulse-propagation problem. Time transformation method employs the concept of temporal mapping of the electric field. Moreover, as it does not make use of the slowly varying envelope approximation (SVEA), it can be applied to pulses of arbitrary widths. Indeed, its predictions agree fully with those of the NLS equation as well as the FDTD technique. Time transformation method is often faster than the FDTD because it does not require the spatial step size to be a small fraction of the wavelength of light. However, since the time transformation directly maps the electric field, temporal step size must be a small fraction of $1 / \omega_{0}$ for an electric field oscillating at $\omega_{0}$. This feature makes the use of the time transformation method time consuming for relatively long pulses with many optical cycles. One might ask whether the time transformation method can be applied to pulse envelopes after removing the fast-oscillating optical carrier so that it can be useful for propagating relatively long pulses? This appendix shows that 
the basic idea behind time transformation method can be easily extended to pulse envelopes.

\section{A.1 Extension to pulse envelopes}

In the standard time transformation method, the input and output electric fields are related as:

$$
E_{\text {out }}(t)=\int_{-\infty}^{\infty} h\left[t-t^{\prime}-T_{d}\left(t^{\prime}\right)\right] E_{\text {in }}\left(t^{\prime}\right) \mathrm{d} t^{\prime},
$$

where $T_{d}$ represents the transit time delay. Consider the propagation of a pulse from the input plane from $z=z_{0}$ to the output plane $z=z_{0}+L$. One can then relate the input electric field to its envelope as

$$
E_{\text {in }}(t)=\frac{1}{2}\left(A_{\text {in }}(t) \exp \left[i\left(\beta_{0} z_{0}-\omega_{0} t\right)\right]+\text { c.c. }\right),
$$

where $\omega_{0}$ is the carrier frequency and $\beta_{0}=n_{0} \omega_{0} / c$ is the propagation constant at this frequency. The output electric field can also be related to its envelope as

$$
E_{\text {out }}(t)=\frac{1}{2}\left(A_{\text {out }}(t) \exp \left\{i\left[\beta_{0}\left(z_{0}+L\right)-\omega_{0} t\right]\right\}+c c .\right) .
$$

One can now relate the input and output pulse envelopes. Substituting Eq. (A.2) into Eq. (A.1) and making use of Eq. (A.3), one obtains

$$
\begin{aligned}
A_{\text {out }}(t) & =e^{-i \beta_{0} L} \int_{-\infty}^{\infty}\left[A_{\text {in }}\left(t^{\prime}\right) e^{i \omega_{0} T_{d}\left(t^{\prime}\right)}\right] \times \\
& h\left[t-t^{\prime}-T_{d}\left(t^{\prime}\right)\right] \exp \left\{i \omega_{0}\left[t-t^{\prime}-T_{d}\left(t^{\prime}\right)\right]\right\} \mathrm{d} t^{\prime} .
\end{aligned}
$$


Although this equation appears quite complicated, it can be simplified considerably by a temporal mapping through the transformation

$$
t_{1}=t^{\prime}+T_{d}\left(t^{\prime}\right)
$$

Converting the integral in Eq. (A.4) from the variable $t^{\prime}$ to $t_{1}$, one obtains

$$
A_{\text {out }}(t)=\int_{-\infty}^{\infty} A\left(t_{1}\right)\left[h\left(t-t_{1}\right) e^{i \omega_{0}\left(t-t_{1}\right)}\right] J\left(t_{1}\right) \mathrm{d} t_{1},
$$

where the Jacobian of the transformation is given by $J=d t^{\prime} / d t_{1}$ and $A\left(t_{1}\right)$ is defined as

$$
A\left(t_{1}\right)=A_{\text {in }}\left(t^{\prime}\right) \exp \left[i \omega_{0} T_{d}\left(t^{\prime}\right)-i \beta_{0} L\right],
$$

Two features of Eq. (A.6) are noteworthy. First, it is in the form of a convolution that can be performed relatively fast in the Fourier domain. Second, the phase factor associated with the impulse response function $h(t)$ in Eq. (A.6) shifts the frequency response function $H(\omega)$ by the carrier frequency $\omega_{0}$. This makes sense because the pulse envelope whose spectrum peaks at $\omega=0$ is considered here.

Equation (A.6) shows how the time transformation method works when applied to pulse envelopes. The effect of medium nonlinearity are contained in the definition of $A\left(t_{1}\right)$ in Eq. (A.7). Physically, $T_{d}\left(t^{\prime}\right)$ represents the delay caused by the medium and $\omega_{0} T_{d}\left(t^{\prime}\right)$ represents the phase shift induced by this delay. The presence of $\beta_{0} L$ in Eq. (A.7) is related to the fact that $T_{d}$ includes both the linear and nonlinear delays. As discussed later, this factor disappears when the linear delay is taken into account. The main advantage of Eq. (A.6) is that the temporal step size in performing the 
convolution numerically has to be a small fraction of the pulse width. In particular, it is not constrained to be a small fraction of the optical cycle, a requirement of the standard time transformation method that deals directly with the transformation of the electric field. This feature makes the time transformation method suitable for multi-cycle optical pulses.

\section{A.2 Application to a nonlinear dispersive medium}

As a specific example, one can apply the preceding general analysis to the specific case of a dispersive Kerr medium whose the refractive index can be expressed as

$$
n(\omega, I)=n_{0}(\omega)+n_{2} I
$$

where $n_{0}(\omega)$ is the frequency-dependent linear part of the refractive index, $n_{2}$ is the Kerr coefficient and $I(t)$ is the optical intensity of the electromagnetic field propagating through the Kerr medium. The transit time can be consequently divided into two parts as $T_{d}=T_{l}+T_{n l}$, where $T_{l}=n_{0}\left(\omega_{0}\right) L / c$ is the linear delay and $T_{n l}=n_{2} I L / c$ is the nonlinear part. Using this expression for $T_{d}$ and substituting it into Eq. (A.8), one can find that the linear phase shift is canceled out by the linear time delay term since $\omega_{0} T_{l}=\beta_{0} L$. As a result, one obtains the following simple expression for the transformation of the pulse envelope by the Kerr medium:

$$
A\left(t_{1}\right)=A_{\text {in }}\left(t^{\prime}\right) e^{i \omega_{0} T_{n l}\left(t^{\prime}\right)} .
$$


Equation (A.9) shows that the Kerr medium affects the pulse envelope in two ways. First, the time-dependent phase factor on the right side of Eq. (A.9) is just the usual nonlinear phase shift related to self-phase modulation (SPM). Indeed, one can write it in the form

$$
\phi_{n l}\left(t^{\prime}\right)=\omega_{0} T_{n l}\left(t^{\prime}\right)=\gamma I\left(t^{\prime}\right) L
$$

where $\gamma=\omega_{0} n_{2} / c$ is the nonlinear parameter [59]. Clearly, the optical pulse modulates its own phase as it passes through a nonlinear Kerr medium because of an intensitydependent refractive index. The second impact of the Kerr nonlinearity is a distortion of the pulse envelope caused by the nonlinear temporal mapping in Eq. (A.5). This is related to the well-known phenomenon of self-steepening. The standard NLS equation ignores self-steepening and incorporates it for short pulses by adding an additional term. In time transformation method, both the SPM and self-steepening are caused by the same Kerr nonlinearity and occur simultaneously. Physically, self-steepening is related to changes in the relative spacing and duration of various temporal slices of the pulse envelope occurring as an optical pulse propagates through the Kerr medium.

Depending on the magnitude of the nonlinearity and the pulse width $T_{0}$, this pulse-shape distortion can be significant or nearly invisible. It is easy to deduce the condition, $T_{n l} \ll T_{0}$, under which pulse-shape distortions are negligible. This condition states that the additional nonlinear delay $T_{n l}$ caused by the reduced speed in the central part of the pulse is a negligible fraction of the pulse width $T_{0}$. For sufficiently short pulses such that $T_{n l} \sim T_{0}$, pulse-shape distortion can become so 
large that they lead to shock formation [100].

For numerical implementation of the time transformation method, one needs to know the functional form of the impulse response function $h(t)$ to take into account the dispersive effects. It is easy to deduce from Eq. (A.1) that the Fourier transform of $h(t)$, or the frequency response function $\tilde{H}(\omega)$ of the medium, is given by

$$
\tilde{H}(\omega)=\exp [i n(\omega) \omega L / c]=\exp [i \beta(\omega) L],
$$

where $\beta(\omega)=n(\omega) \omega / c$ is the propagation constant at frequency $\omega$. Although one can find $h(t)$ by taking the inverse Fourier transform of $\tilde{H}(\omega)$, it is not required in practice. The reason is that the integration in Eq. (A.6) is performed in the frequency domain where a convolution becomes just the product of the two functions being convolved, i.e.,

$$
A_{\text {out }}(t)=\mathcal{F}^{-1}\left(\tilde{H}\left(\omega-\omega_{0}\right) \mathcal{F}\left[A\left(t_{1}\right)\right]\right),
$$

where $\mathcal{F}$ denotes Fourier-transform operation. An important issue to consider is the choice of $L$ in Eq. (A.10). Since $T_{n l}$ depends on the pulse shape that itself changes with propagation, one cannot make $L$ very large. In other words, the total length over which the optical pulse is to be propagated must be divided into multiple sections such that the length $\Delta z$ of each section is relatively small. In practice, $\Delta z$ is chosen such that the nonlinear phase $\phi_{n l} \ll 1$.

As a numerical example, the propagation of a multi-cycle pulse at a carrier frequency of $200 \mathrm{THz}$ (wavelength $1500 \mathrm{~nm}$ ) propagating inside a nonlinear dispersive medium is considered. Specifically, the input envelope is taken to be $A_{\text {in }}(t)=$ 


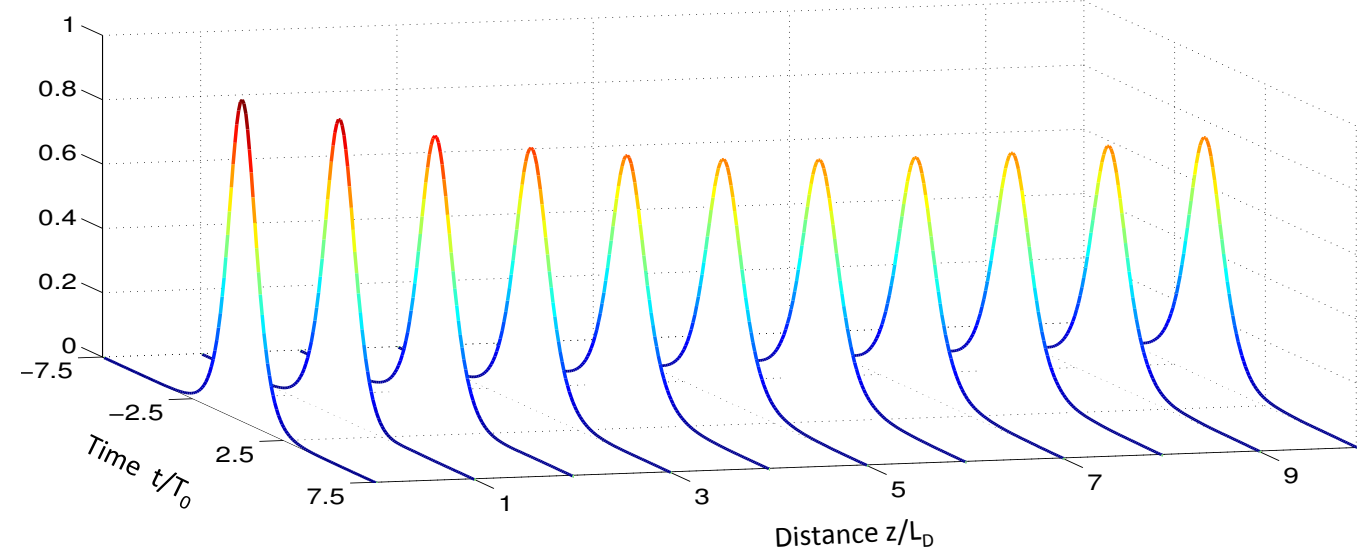

Figure A.1: Propagation of a multi-cycle soliton-like pulse over 10 dispersion lengths inside a Kerr medium whose dispersion is characterized by the Lorentz model.

$A_{0} \operatorname{sech}\left(t / T_{0}\right)$ with $T_{0}=20$ fs. Chromatic dispersion of the medium is included using the single-resonance Lorentz model used in section 8.3 with identical parameters. The peak amplitude $A_{0}$ is chosen to satisfy the soliton-formation condition [59] and is found using $n_{2} A_{0}^{2}=0.0046$. In the absence of self-steepening and higher-order dispersive effects, such a pulse would maintain its shape during propagation.

Figure A.1 shows the evolution of pulse envelope over 10 dispersion lengths (about $500 \mu \mathrm{m})$ using the time transformation method that automatically includes selfsteepening and all higher-order dispersive effects. As seen there, the pulse envelope broadens, becomes asymmetric, and its center slightly shifts because of multiple higher-order effects. Nevertheless, it continues to exhibit soliton-like features, suggesting that even 20 -fs pulses can be transported over tens of dispersion lengths without significant broadening and shape distortion. 
The envelope time transformation method discussed here is quite different conceptually from the approach based on the standard NLS equation [Eq. (2.21)]. The two methods resemble in their numerical implementations, inasmuch as both of them employ the fast-Fourier-transform (FFT) algorithm and require propagation using multiple sections. One may thus ask if anything is gained by employing the time transformation. The answer is related to the approximate nature of the NLS equation. First, Eq. (2.21) includes dispersion through the Taylor expansion of $\beta(\omega)$ around the carrier frequency $\omega_{0}$ and retains only the $\beta_{2}$ term that is second-order in $\omega-\omega_{0}$. Second, the self-steepening effects are absent from this equation. If one uses this equation for the 20-fs pulse used for Figure 1, the pulse envelope will not change at all over the 10 dispersion lengths.

Both of these limitations can be overcome by employing the so-called generalized NLS equation which adds higher-order dispersive terms in addition to the selfsteepening term [59]. Indeed, when one uses the generalized NLS equation, one could obtain the results identical to those shown in Figure A.1 only when all higher-order dispersion terms are included. Another advantage of the time transformation method is that it can be applied to a medium with an arbitrary form of the nonlinear response, as long as one can calculate the delay time $T_{d}$ appearing in Eq. (A.5). Even the delayed nonlinear response coming from a Raman-type nonlinearity can be handled by the time transformation method.

The main reason that the NLS equation, or its generalized version, is widely used 
in practice is that it deals with pulse envelopes and can be solved numerically faster using the split-step Fourier method, compared with the FDTD solution of Maxwell's equations. This appendix shows that the time transformation method can also be applied to pulse envelopes, and its use may be preferable in certain situations. One might ask how the speed and accuracy of the time transformation method compare to those of the NLS equation. A series of calculations involving different combinations of propagation distances, nonlinear and dispersive parameters, and pulse shapes is performed and it is found that the two approaches agree in all cases. The computing times are comparable for the two methods for realizing the same accuracy. 


\section{B Numerical Code for The Time Transformation Method}

This Appendix includes a MATLAB m-file that shows the numerical implementation of the time transformation method for solving the propagation problem of an optical pulse inside a nonlinear dispersive medium. Both the Kerr-type and Raman-type nonlinearity are included, and dispersion is included through a Taylor-expansion of the wave vector. The algorithm is essentially a split-step Fourier algorithm, where the whole medium is divided into small sections and the evolution of the pulse is considered from step to step. Nonlinearity is calculated in time domain through time transformation, which is implemented in MATLAB using its own build-in function "interp1". Dispersion is calculated in the frequency domain using Fourier transforms.

\%time transformation code for nonlinear dispersive pulse propagation.

\%written by Yuzhe Xiao

\% Feb 11, 2014 
\%The Institute of Optics, University of Rochester

clear all;

$\% \% \% \% \% \% \% \% \%$ Input simulation parameters

$\mathrm{LD}=1 ; \quad \%$ dispersion length, normalized to one

gamma $=9 ; \quad \%$ gamma, normalized that "gamma $=\mathrm{N}^{\wedge} 2$ ", $\mathrm{N}$, soliton order

delta $2=-1 ; \quad \%$ normalized beta 2 parameter beta $2 / \mathrm{T}^{\wedge} 2$

delta3 $=0.0 ; \quad \%$ normalized beta3 parameter beta3 $/(6 * \mathrm{~T} 0 *$ abs $($ beta 2$))$

$\mathrm{L}=3 * \mathrm{LD} ; \quad \%$ propagation length

$\mathrm{NN}=1000 ; \quad \%$ number of step size

$\mathrm{dL}=\mathrm{L} / \mathrm{NN} ; \quad \%$ length of each small section

$\mathrm{T} 0=1 ; \quad \%$ actual pulse width, in unit of fs

$\mathrm{s}=1 / \mathrm{T} 0 / 0.4 / \mathrm{pi} ; \quad \%$ self-steepening factor, with the choice of $\mathrm{f} 0=200 \mathrm{THz}$

$\mathrm{f} 0=1 /\left(\mathrm{s}^{*} 2 * \mathrm{pi}\right) ; \quad \%$ carrier frequency, normalized to pulse width

$\% \% \% \% \% \% \% \% \% \%$ input field

$\mathrm{dt}=1 / \mathrm{f} 0 / 40 ; \quad \%$ in order to resove the phase changes

$\mathrm{N}=2 *$ round $(100 / \mathrm{dt}) ; \quad \%$ total number of points

$\mathrm{t}=(-\mathrm{N} / 2: \mathrm{N} / 2-1)^{*} \mathrm{dt} ; \quad \%$ time frame: time frame range : $-100^{*} \mathrm{~T} 0: 100 * \mathrm{~T} 0$

$\mathrm{df}=1 /(\mathrm{dt} * \mathrm{~N})$

$\mathrm{f}=(-\mathrm{N} / 2: \mathrm{N} / 2-1)^{*} \mathrm{df} ; \quad \%$ frequency grid 
$\%$ coefficient in FFT and iFFT

$\mathrm{AA}=\operatorname{zeros}(1, \mathrm{~N})$

for $\mathrm{jj}=1: \mathrm{N}$

$\mathrm{AA}(\mathrm{jj})=(-1)^{\wedge}(\mathrm{jj}-1) ;$

end

power $=\operatorname{sech}(\mathrm{t})$;

phase $=2^{*} \mathrm{pi}^{*} \mathrm{f} 0^{*} \mathrm{t}$;

Ein $=$ power. ${ }^{*} \exp \left(-1 i^{*}\right.$ phase $)$

Fin $=$ AA. $*$ fftshift $($ ifft $(($ Ein $))) * d t * N$

$\%$ - Input 3D data

zstep $=50 ; \%$ Define the no of z step

$\mathrm{ng}=\mathrm{NN} / \mathrm{zstep}$; \% The number of interval where I want to store data for 3D.

E_3d $(1,:)=\operatorname{abs}($ Ein).^2; \% Intensity in time domain

F_3d(1,:)=abs(Fin).^2; \% spectrum au unit

$\% \% \% \% \% \% \% \% \% \% \% \%$ Defination of Raman response function

$\mathrm{fR}=0.245 ;$

$\operatorname{tau} 1=12.2 / \mathrm{T} 0$

$\operatorname{tau} 2=32 / \mathrm{T} 0$;

tau_b=96/T0;

$\mathrm{fa}=0.75$; 
$\mathrm{fb}=0.21$;

$\mathrm{fc}=0.04$

$\mathrm{h}=\operatorname{zeros}(1, \mathrm{~N})$;

for $\mathrm{ii}=(\mathrm{N}) / 2+1: \mathrm{N}$;

$\mathrm{h}(\mathrm{ii})=(\mathrm{fa}+\mathrm{fc}) *\left(\operatorname{tau} 1^{\wedge} 2+\operatorname{tau} 2^{\wedge} 2\right) /\left(\operatorname{tau} 1^{*} \operatorname{tau} 2^{\wedge} 2\right)^{*} \exp (-\mathrm{t}(\mathrm{ii}) / \operatorname{tau} 2)^{*} \sin (\mathrm{t}(\mathrm{ii}) / \operatorname{tau} 1)$

$+\mathrm{fb}^{*}\left(\left(2^{*}\right.\right.$ tau_b-t(ii))/tau_b^2)*exp(-t(ii)/tau_b);

end

$\mathrm{H}=\mathrm{AA} .{ }^{*} \mathrm{fftshift}(\mathrm{ifft}((\mathrm{h}))) .{ }^{*} \mathrm{~N}^{*} \mathrm{dt} ; \%$ Frequency response of Raman

$\%$ Medium dispersion

$\mathrm{D}=\exp \left(0.5 \mathrm{i}^{*} \operatorname{delta} 2{ }^{*} 4^{*} \mathrm{pi}^{\wedge} 2^{*}(\mathrm{f}-\mathrm{f} 0) .{ }^{*} 2^{*} \mathrm{dL}+1 \mathrm{i}^{*} \mathrm{delta3} 3^{*} 8^{*} \mathrm{pi}^{\wedge} 3^{*}(\mathrm{f}-\mathrm{f} 0) .{ }^{\wedge} 3^{*} \mathrm{dL}\right)$

$\% \% \% \% \% \% \% \% \% \% \% \%$ begin of the main calculation

$\mathrm{E}=\operatorname{Ein} ;$

tic

for $\mathrm{n}=1: \mathrm{NN}$

n

$\mathrm{T} \_$Kerr $=\mathrm{s}^{*}$ gamma*dL*abs(E).^2; \% nonlinear time shift: Kerr nonlinearity

conv_fn=H.*AA.*ftshift(ifft(abs(E).^2));

convl=AA.*fftshift(fft $\left(\left(\right.\right.$ conv_fn $\left.\left._{)}\right)\right)$;

T_Raman $=$ real $\left(\mathrm{s}^{*}\right.$ gamma*dL*convl); \% nonlinear time shift: Raman nonlinearity $\mathrm{T}=(1-\mathrm{fR}){ }^{*} \mathrm{~T} \_$Kerr $+\mathrm{fR}{ }^{*} \mathrm{~T} \_$Raman; \% total nonlinear time shift 
$\mathrm{E}=\operatorname{interp} 1(\mathrm{t}+\mathrm{T}, \mathrm{E}, \mathrm{t}$, 'spline','extrap')./(1+deriv1(T,dt)); \% time transformation $\mathrm{F}=\mathrm{AA} \cdot *^{*} \mathrm{ft} \operatorname{shift}(\operatorname{ifft}((\mathrm{E})))$

$\mathrm{E}=\mathrm{AA} \cdot{ }^{*} \mathrm{fftshift}\left(\mathrm{fft}\left(\left(\mathrm{F} \cdot{ }^{*} \mathrm{D}\right)\right)\right) ; \%$ inclusion of medium dispersion

if $\bmod (\mathrm{n}, \mathrm{ng})==0$;

E_3d((n/ng+1),::)=abs(E).^2; \% temporal power

F_3d((n/ng+1),: $)=\operatorname{abs}\left(\right.$ AA.*fftshift $\left.(i f f t((E))) * \mathrm{~N}^{*} \mathrm{dt}\right)$ ` $^{\wedge} ; \%$ spectral power

end

end

toc

$\% \% \% \% \% \% \% \% \% \% \% \% \% \%$ end of the main calculation

Eout $=\mathrm{E}$;

Fout $=$ AA. $*$ fftshift $($ ifft $(($ Eout $))) * \mathrm{dt}^{*} \mathrm{~N}$;

$\% \% \% \% \% \% \% \% \% \% \% \% \% \%$ plotting the output pulse shape and spectrum

figure;

$\operatorname{subplot}(2,1,1)$;

plot(t,abs(Ein).^2,'g','LineWidth',1);hold on;

$\operatorname{plot}(\mathrm{t}, \mathrm{abs}($ Eout).^2,'r','LineWidth',1);

$\operatorname{axis}\left(\left[\begin{array}{llll}-5 & 70 & 0 & \mathrm{inf}\end{array}\right]\right)$

title('Pulse shape (Time transformation)','fontsize',12);

set(gca,'fontsize',12) 
$\operatorname{subplot}(2,1,2)$;

semilogy(f-f0,(abs(Fin)/max(abs(Fin))).`2,'g','LineWidth',1);hold on;

semilogy(f-f0,(abs(Fout)/max(abs(Fin))). `2,'r','LineWidth',1);

$\operatorname{axis}([-3+40.01 \mathrm{inf}])$

title('Spectrum','fontsize',12);

set(gca,'fontsize',12)

$\% \% \% \% \% \% \% \% \% \% \% \% \%$ plotting the evolution of pulse along the propagation

figure

$\%$ colormap $(1-$ jet $)$

$\mathrm{z}=$ linspace $(0, \mathrm{~L}, \mathrm{zstep}+1) ; \%$ normalised distance

subplot $(1,2,1)$;

E_3d $=10^{*} \log 10\left(E \_3 d+e p s\right) ; \%$ convert to $\mathrm{dB}$ units

$\operatorname{pmax}=\max \left(\max \left(\mathrm{E} \_3 \mathrm{~d}\right)\right) ; \% \max$ value for scaling plot

pcolor $\left(\mathrm{t}, \mathrm{z}, \mathrm{E} \_3 \mathrm{~d}\right)$;

$\operatorname{caxis}([\operatorname{pmax}-50, \operatorname{pmax}]) ; \operatorname{xlim}([-10,60]) ;$ shading interp;

set(gca,'FontSize', 12); \%title('(a)')

xlabel('Time (t/T_0)'); ylabel('Distance (z/L_D)')

$\operatorname{subplot}(1,2,2)$

F_3d $=10^{*} \log 10\left(\mathrm{~F} \_3 \mathrm{~d}+\mathrm{eps}\right) ; \%$ convert to $\mathrm{dB}$ units 
$\operatorname{pmax}=\max \left(\max \left(\mathrm{F} \_3 \mathrm{~d}\right)\right) ; \%$ max value for scaling plot pcolor $\left(\mathrm{f}-\mathrm{f} 0, \mathrm{z}, \mathrm{F} \_3 \mathrm{~d}\right)$;

caxis $([\operatorname{pmax}-50, \operatorname{pmax}]) ; \operatorname{xlim}([-3,3])$; shading interp; set(gca,'FontSize', 12); \%title('(a)') xlabel('(nu-nu _0)T_0'); \%ylabel('Distance (z/L_D)') 


\section{Bibliography}

[1] M. Notomi and S. Mitsugi, "Wavelength conversion via dynamic refractive index tuning of a cavity," Phy. Rev. A, vol. 73, no. 5, 2006.

[2] S. F. Preble, Q. Xu, and M. Lipson, "Changing the colour of light in a silicon resonator," Nature Photon., vol. 1, no. 5, pp. 293-296, 2007.

[3] T. Kampfrath, D. Beggs, T. White, A. Melloni, T. Krauss, and L. Kuipers, "Ultrafast adiabatic manipulation of slow light in a photonic crystal," Phy. Rev. A, vol. 81, no. 4, p. 043837, 2010.

[4] M. Born and E. Wolf, Principles of Optics. Cambridge, 1999.

[5] H. Goldstein, Classical Mechanics. Pearson, 1962, vol. 4.

[6] M. Mansuripur, "Radiation pressure and the linear momentum of the electromagnetic field in magnetic media," Opt. Express, vol. 15, no. 21, pp. $13502-$ $13518,2007$.

[7] F. R. Morgenthaler, "Velocity modulation of electromagnetic waves," IEEE Trans. Microwave Theory Tech., vol. 6, no. 2, pp. 167-172, 1958. 
[8] R. Fante, "Transmission of electromagnetic waves into time-varying media," IEEE Trans. Antennas Propagat., vol. 19, no. 3, pp. 417-424, 1971.

[9] R. Landauer, "Velocity modulation of propagating waves," J. of Appl. Phy., vol. 34, no. 7, pp. 1893-1896, 1963.

[10] C.-L. Jiang, "Wave propagation and dipole radiation in a suddenly created plasma," IEEE Trans. Antennas Propagat., vol. 23, no. 1, pp. 83-90, 1975.

[11] T. Ruiz, C. Wright, and J. Smith, "Characteristics of electromagnetic waves propagating in time varying media," IEEE Trans. Antennas Propagat., vol. 26, no. 2, pp. 358-361, 1978.

[12] J. C. AuYeung, "Phase-conjugate reflection from a temporal dielectric boundary," Opt. Lett., vol. 8, no. 3, pp. 148-150, 1983.

[13] D. K. Kalluri, "On reflection from a suddenly created plasma half-space: Transient solution," IEEE Trans. Plasma Sci., vol. 16, no. 1, pp. 11-16, 1988.

[14] S. Wilks, J. Dawson, and W. Mori, "Frequency up-conversion of electromagnetic radiation with use of an overdense plasma," Phy. Rev. Lett., vol. 61, no. 3, p. $337,1988$.

[15] D. Kalluri, "Effect of switching a magnetoplasma medium on a traveling wave: longitudinal propagation," IEEE Trans. Antennas Propagat., vol. 37, no. 12, pp. 1638-1642, 1989.

[16] E. Esarey, G. Joyce, and P. Sprangle, "Frequency up-shifting of laser pulses by copropagating ionization fronts," Phy. Rev. A, vol. 44, no. 6, p. 3908, 1991. 
[17] F. A. Harfoush and A. Taflove, "Scattering of electromagnetic waves by a material half-space with a time-varying conductivity," IEEE Trans. Antennas Propagat., vol. 39, no. 7, pp. 898-906, 1991.

[18] M. Cirone, K. Rzazewski, and J. Mostowski, "Photon generation by timedependent dielectric: a soluble model," Phy. Rev. A, vol. 55, no. 1, pp. 62-66, 1997.

[19] V. Berezhiani, S. Mahajan, and R. Miklaszewski, "Frequency up-conversion and trapping of ultrashort laser pulses in semiconductor plasmas," Phy. Rev. A, vol. 59, no. 1, pp. 859-864, 1999.

[20] M. Bakunov, V. Gildenburg, S. Zhukov, and N. Zharova, "Adiabatic frequency shifting of a surface wave guided by a time-varying plasma structure," Phys. Plasmas, vol. 7, p. 1035, 2000.

[21] M. Bakunov and I. Grachev, "Energetics of electromagnetic wave transformation in a time-varying magnetoplasma medium," Phy. Rev. E, vol. 65, no. 3, p. 036405, 2002.

[22] S. Kuo, "Frequency up-conversion of microwave pulse in a rapidly growing plasma," Phy. Rev. Lett., vol. 65, no. 8, p. 1000, 1990.

[23] C. J. Joshi, C. Clayton, K. Marsh, D. Hopkins, A. Sessler, and D. Whittum, "Demonstration of the frequency upshifting of microwave radiation by rapid plasma creation," IEEE Trans. Plasma Sci., vol. 18, no. 5, pp. 814-818, 1990.

[24] B. Penetrante, J. Bardsley, W. M. Wood, C. Siders, and M. C. Downer, 
"Ionization-induced frequency shifts in intense femtosecond laser pulses," $J$. Opt. Soc. Am. B, vol. 9, no. 11, pp. 2032-2040, 1992.

[25] S. P. Kuo and A. Ren, "Experimental study of wave propagation through a rapidly created plasma," IEEE Trans. Plasma Sci., vol. 21, no. 1, pp. 53-56, 1993.

[26] N. Yugami, T. Niiyama, T. Higashiguchi, H. Gao, S. Sasaki, H. Ito, and Y. Nishida, "Experimental observation of short-pulse upshifted frequency microwaves from a laser-created overdense plasma," Phy. Rev. E, vol. 65, no. 3, p. $036505,2002$.

[27] I. Geltner, Y. Avitzour, and S. Suckewer, "Picosecond pulse frequency upshifting by rapid free-carrier creation in ZnSe," Appl. Phy. Lett., vol. 81, no. 2, pp. $226-228,2002$.

[28] Y. Avitzour, I. Geltner, and S. Suckewer, "Laser pulse frequency shifting by ionization and recombination fronts in semiconductor plasma," J. Phys. B: At. Mol. Opt. Phys., vol. 38, no. 7, p. 779, 2005.

[29] A. Nishida, N. Yugami, T. Higashiguchi, T. Otsuka, F. Suzuki, M. Nakata, Y. Sentoku, and R. Kodama, "Experimental observation of frequency upconversion by flash ionization," Appl. Phy. Lett., vol. 101, no. 16, pp. $161118-$ $161118,2012$.

[30] E. J. Reed, M. Soljačić, and J. D. Joannopoulos, "Color of shock waves in photonic crystals," Phy. Rev. Lett., vol. 90, no. 20, p. 203904, 2003. 
[31] — - "Reversed Doppler effect in photonic crystals," Phy. Rev. Lett., vol. 91, no. 13, p. 133901, 2003.

[32] M. F. Yanik and S. Fan, "Stopping light all optically," Phy. Rev. Lett., vol. 92, no. 8, p. 083901, 2004.

[33] — - "Time reversal of light with linear optics and modulators," Phy. Rev. Lett., vol. 93, no. 17, p. 173903, 2004.

[34] Z. Gaburro, M. Ghulinyan, F. Riboli, L. Pavesi, A. Recati, I. Carusotto et al., "Photon energy lifter." Opt. Express, vol. 14, no. 16, p. 7270, 2006.

[35] M. Notomi, H. Taniyama, S. Mitsugi, and E. Kuramochi, "Optomechanical wavelength and energy conversion in high-q double-layer cavities of photonic crystal slabs," Phy. Rev. Lett., vol. 97, no. 2, p. 023903, 2006.

[36] A. Khorshidahmad and A. G. Kirk, "Wavelength conversion by dynamically reconfiguring a nested photonic crystal cavity," Opt. Express, vol. 18, no. 8, pp. $7732-7742,2010$.

[37] M. W. McCutcheon, A. G. Pattantyus-Abraham, G. W. Rieger, and J. F. Young, "Emission spectrum of electromagnetic energy stored in a dynamically perturbed optical microcavity," Opt. Express, vol. 15, no. 18, pp. 11472-11480, 2007.

[38] T. Tanabe, M. Notomi, H. Taniyama, and E. Kuramochi, "Dynamic release of trapped light from an ultrahigh-Q nanocavity via adiabatic frequency tuning," Phy. Rev. Lett., vol. 102, no. 4, p. 043907, 2009. 
[39] A. W. Elshaari and S. F. Preble, "Active optical isolator using adiabatic wavelength conversion in microcavities," in Frontiers in Optics. Optical Society of America, 2009, p. FThU4.

[40] J. R. Zurita-Sánchez, P. Halevi, and J. C. Cervantes-González, "Reflection and transmission of a wave incident on a slab with a time-periodic dielectric function $\epsilon(t), "$ Phy. Rev. A, vol. 79, no. 5, p. 053821, 2009.

[41] J. R. Zurita-Sánchez, J. Abundis-Patiño, and P. Halevi, "Pulse propagation through a slab with time-periodic dielectric function $\varepsilon(t), "$ Opt. Express, vol. 20, no. 5, pp. 5586-5600, 2012.

[42] B. A. Daniel, D. N. Maywar, and G. P. Agrawal, "Efficient adiabatic wavelength conversion in Gires-Tournois resonators," Opt. Lett., vol. 36, no. 21, pp. 41554157, 2011.

[43] S. Preble, L. Cao, A. Elshaari, A. Aboketaf, and D. Adams, "Single photon adiabatic wavelength conversion," Appl. Phy. Lett., vol. 101, no. 17, p. 171110, 2012.

[44] F. Biancalana, A. Amann, A. V. Uskov, and E. P. OReilly, "Dynamics of light propagation in spatiotemporal dielectric structures," Phy. Rev. E, vol. 75, no. 4, p. $046607,2007$.

[45] J. Mendonça, A. Guerreiro, and A. M. Martins, "Quantum theory of time refraction," Phy. Rev. A, vol. 62, no. 3, p. 033805, 2000.

[46] J. T. Mendonça, Theory of photon acceleration. CRC Press, 2010. 
[47] J. Mendonca and P. Shukla, "Time refraction and time reflection: two basic concepts," Phys. Scripta, vol. 65, no. 2, p. 160, 2002.

[48] P. Dong, S. F. Preble, J. T. Robinson, S. Manipatruni, and M. Lipson, "Inducing photonic transitions between discrete modes in a silicon optical microcavity," Phy. Rev. Lett., vol. 100, no. 3, p. 033904, 2008.

[49] B. A. Daniel, D. N. Maywar, and G. P. Agrawal, "Dynamic mode theory of optical resonators undergoing refractive index changes," J. Opt. Soc. Am. B, vol. 28, no. 9, pp. 2207-2215, 2011.

[50] Y. Tanaka, J. Upham, T. Nagashima, T. Sugiya, T. Asano, and S. Noda, "Dynamic control of the $\mathrm{Q}$ factor in a photonic crystal nanocavity," Nature Mat., vol. 6, no. 11, pp. 862-865, 2007.

[51] M. Notomi, T. Tanabe, A. Shinya, E. Kuramochi, H. Taniyama, S. Mitsugi, and M. Morita, "Nonlinear and adiabatic control of high-Q photonic crystal nanocavities," Opt. Express, vol. 15, no. 26, pp. $17458-17481,2007$.

[52] Q. Lin, T. Johnson, C. Michael, and O. Painter, "Adiabatic self-tuning in a silicon microdisk optical resonator," Opt. Express, vol. 16, no. 19, pp. 14801$14811,2008$.

[53] T. Kampfrath, D. M. Beggs, T. F. Krauss, and L. K. Kuipers, "Complete response characterization of ultrafast linear photonic devices," Opt. Lett., vol. 34, no. 21, pp. 3418-3420, 2009.

[54] J. Upham, Y. Tanaka, T. Asano, and S. Noda, "On-the-fly wavelength con- 
version of photons by dynamic control of photonic waveguides," Appl. Phy. Express, vol. 3, no. 6, p. 2001, 2010.

[55] B. Chen, B. Gao, C. Ge, and J. Li, "Accurate solution and characteristics for electromagnetic wave propagation in time-varying media," Mod. Appl. Sci., vol. 3, no. 10, p. P68, 2009.

[56] Y. Xu, Y. Li, R. K. Lee, and A. Yariv, "Scattering-theory analysis of waveguideresonator coupling," Phy. Rev. E, vol. 62, no. 5, p. 7389, 2000.

[57] R. W. Boyd, Nonlinear Optics. Academic press, 2003.

[58] F. Shimizu, "Frequency broadening in liquids by a short light pulse," Phy. Rev. Lett., vol. 19, no. 19, p. 1097, 1967.

[59] G. P. Agrawal, Nonlinear Fiber Optics. Academic Press, 2012.

[60] F. DeMartini, C. Townes, T. Gustafson, and P. Kelley, "Self-steepening of light pulses," Phy. Rev., vol. 164, no. 2, p. 312, 1967.

[61] N. Tzoar and M. Jain, "Self-phase modulation in long-geometry optical waveguides," Phy. Rev. A, vol. 23, no. 3, p. 1266, 1981.

[62] D. Anderson and M. Lisak, "Nonlinear asymmetric self-phase modulation and self-steepening of pulses in long optical waveguides," Phy. Rev. A, vol. 27, no. 3, p. 1393, 1983.

[63] R. A. Fisher and W. Bischel, "The role of linear dispersion in plane-wave selfphase modulation," Appl. Phy. Lett., vol. 23, no. 12, pp. 661-663, 1973. 
[64] J.-C. Diels and W. Rudolph, Ultrashort laser pulse phenomena. Academic press, 2006.

[65] N. Zabusky, "Interaction of solitions in a collisionless plasma and the recurrence of initial states," Phy. Rev. Lett., vol. 15, no. 6, pp. 240-243, 1965.

[66] K. E. Oughstun and N. A. Cartwright, "On the Lorentz-Lorenz formula and the Lorentz model of dielectric dispersion," Opt. Express, vol. 11, no. 13, pp. 1541-1546, 2003.

[67] G. P. Agrawal, Fiber-Optic Communication Systems. Wiley, 2010.

[68] Q. Lin and G. P. Agrawal, "Raman response function for silica fibers," Opt. Lett., vol. 31, no. 21, pp. 3086-3088, 2006.

[69] K. J. Blow and D. Wood, "Theoretical description of transient stimulated Raman scattering in optical fibers," IEEE J. Quantum Electron., vol. 25, no. 12, pp. 2665-2673, 1989.

[70] F. M. Mitschke and L. F. Mollenauer, "Discovery of the soliton self-frequency shift," Opt. Lett., vol. 11, no. 10, pp. 659-661, 1986.

[71] J. P. Gordon, "Theory of the soliton self-frequency shift," Opt. Lett., vol. 11, no. 10 , pp. $662-664,1986$.

[72] C.-T. Chen, Linear System Theory and Design. Oxford University Press, 1998.

[73] J. W. Goodman, Introduction to Fourier Optics. Roberts and Company Publishers, 2005. 
[74] S. J. Orfanidis, Electromagnetic Waves and Antennas. Rutgers University Press, 2002.

[75] Y. Xiao, G. P. Agrawal, and D. N. Maywar, "Spectral and temporal changes of optical pulses propagating through time-varying linear media," Opt. Lett., vol. 36, no. 4, pp. 505-507, 2011.

[76] Y. Xiao, D. N. Maywar, and G. P. Agrawal, "Optical pulse propagation in dynamic fabry-perot resonators," J. Opt. Soc. Am. B, vol. 28, no. 7, pp. 16851692, 2011.

[77] Y. Xiao, G. P. Agrawal, and D. N. Maywar, "Nonlinear pulse propagation: A time-transformation approach," Opt. Lett., vol. 37, no. 7, pp. 1271-1273, 2012.

[78] Y. Xiao, D. N. Maywar, and G. P. Agrawal, "New approach to pulse propagation in nonlinear dispersive optical media," J. Opt. Soc. Am. B, vol. 29, no. 10, pp. 2958-2963, 2012.

[79] — _ "Propagation of few-cycle pulses in nonlinear kerr media: harmonic generation," Opt. Lett., vol. 38, no. 5, pp. 724-726, 2013.

[80] B. Jalali, D. Solli, and S. Gupta, "Silicon photonics: Silicon's time lens," Nature Photon., vol. 3, no. 1, pp. 8-10, 2009.

[81] E. F. Burmeister, D. J. Blumenthal, and J. E. Bowers, "A comparison of optical buffering technologies," Opt. Switch. Netw., vol. 5, no. 1, pp. 10-18, 2008.

[82] D. Kalluri and V. Goteti, "Frequency shifting of electromagnetic radiation by sudden creation of a plasma slab," J. of Appl. Phy., vol. 72, no. 10, pp. 4575- 
4580, 1992.

[83] G. P. Agrawal, Lightwave Technology: Components and Devices. WileyInterscience, 2004.

[84] S. Harris and O. McDuff, "Theory of FM laser oscillation," IEEE J. Quantum Electron., vol. 1, no. 6, pp. 245-262, 1965.

[85] M. Lawrence, B. Willke, M. Husman, E. Gustafson, and R. Byer, "Dynamic response of a Fabry-Perot interferometer," J. Opt. Soc. Am. B, vol. 16, no. 4, pp. 523-532, 1999 .

[86] M. Rakhmanov, "Doppler-induced dynamics of fields in Fabry-Perot cavities with suspended mirrors," Appl. Opt., vol. 40, no. 12, pp. 1942-1949, 2001.

[87] H. Rohde, J. Eschner, F. Schmidt-Kaler, and R. Blatt, "Optical decay from a Fabry-Perot cavity faster than the decay time," J. Opt. Soc. Am. B, vol. 19, no. 6, pp. 1425-1429, 2002.

[88] D. Redding, M. Regehr, and L. Sievers, "Dynamic models of Fabry-Perot interferometers," Appl. Opt., vol. 41, no. 15, pp. 2894-2906, 2002.

[89] M. Rakhmanov, R. Savage Jr, D. Reitze, and D. Tanner, "Dynamic resonance of light in Fabry-Perot cavities," Phy. Lett. A, vol. 305, no. 5, pp. 239-244, 2002.

[90] A. W. Snyder, D. J. Mitchell, and Y. S. Kivshar, "Unification of linear and nonlinear wave optics," Mod. Phys. Lett. B, vol. 9, no. 23, pp. 1479-1506, 1995.

[91] T. Brabec and F. Krausz, "Nonlinear optical pulse propagation in the single- 
cycle regime," Phy. Rev. Lett., vol. 78, pp. 3282-3285, 1997.

[92] Y. Xiao, D. N. Maywar, and G. P. Agrawal, "Time-transformation approach to pulse propagation in nonlinear dispersive media: Inclusion of delayed nonlinearity," Phy. Rev. A, vol. 87, no. 6, p. 063816, 2013.

[93] D. Skryabin and A. Yulin, "Theory of generation of new frequencies by mixing of solitons and dispersive waves in optical fibers," Phy. Rev. E, vol. 72, no. 1, p. 016619, 2005.

[94] G. Chang, L.-J. Chen, and F. X. Kärtner, "Highly efficient Cherenkov radiation in photonic crystal fibers for broadband visible wavelength generation," Opt. Lett., vol. 35, no. 14, pp. 2361-2363, 2010.

[95] G. Genty, P. Kinsler, B. Kibler, and J. Dudley, "Nonlinear envelope equation modeling of sub-cycle dynamics and harmonic generation in nonlinear waveguides," Opt. Express, vol. 15, no. 9, pp. 5382-5387, 2007.

[96] P. M. Goorjian and A. Taflove, "Direct time integration of maxwells equations in nonlinear dispersive media for propagation and scattering of femtosecond electromagnetic solitons," Opt. Lett., vol. 17, no. 3, pp. 180-182, 1992.

[97] R. Flesch, A. Pushkarev, and J. Moloney, "Carrier wave shocking of femtosecond optical pulses," Phy. Rev. Lett., vol. 76, no. 14, pp. 2488-2491, 1996.

[98] L. Gilles, J. Moloney, and L. Vázquez, "Electromagnetic shocks on the optical cycle of ultrashort pulses in triple-resonance lorentz dielectric media with subfemtosecond nonlinear electronic debye relaxation," Phy. Rev. E, vol. 60, no. 1, 
p. 1051, 1999.

[99] E. Rubino, J. McLenaghan, S. Kehr, F. Belgiorno, D. Townsend, S. Rohr, C. Kuklewicz, U. Leonhardt, F. König, and D. Faccio, "Negative-frequency resonant radiation," Phy. Rev. Lett., vol. 108, no. 25, p. 253901, 2012.

[100] D. Grischkowsky, E. Courtens, and J. Armstrong, "Observation of selfsteepening of optical pulses with possible shock formation," Phy. Rev. Lett., vol. 31, pp. 422-425, 1973. 